\title{
Title: Controlled Sound Field with a Dual Layer Loudspeaker
}

\section{Array}

\section{Running Title: Sound Field Control with a Dual Layer Array}

Mincheol Shin ${ }^{1)^{*}}$, Filippo M. Fazi ${ }^{2)}$, Philip A. Nelson ${ }^{3)}$ and Fabio C. Hirono ${ }^{4)}$

1) M.Shin@soton.ac.uk, +44(0)2380594974, Institute of Sound and Vibration Research, University of Southampton (ISVR), University Road, Highfield, Southampton S017 1BJ, United Kingdom

2) Filippo.Fazi@soton.ac.uk,_44(0)2380594972, Institute of Sound and Vibration Research, University of Southampton (ISVR), University Road, Highfield, Southampton S017 1BJ, United Kingdom

3) P.A.Nelson@ soton.ac.uk,+44(0)2380592259, Institute of Sound and Vibration Research, University of Southampton (ISVR), University Road, Highfield, Southampton S017 1BJ, United Kingdom

4) fch1g10@soton.ac.uk, +44(0)2380594912, Institute of Sound and Vibration Research, University of Southampton (ISVR), University Road, Highfield, Southampton S017 1BJ, United Kingdom

Submitted (January, 2013), First revised (August, 2013) and Second revised (January, 2014) to Journal of Sound and Vibration 


\begin{abstract}
Controlled sound interference has been extensively investigated using a prototype dual layer loudspeaker array comprised of 16 loudspeakers. Results are presented for measures of array performance such as input signal power, directivity of sound radiation and accuracy of sound reproduction resulting from the application of conventional control methods such as minimization of error in mean squared pressure, maximization of energy difference and minimization of weighted pressure error and energy. Procedures for selecting the tuning parameters have also been introduced. With these conventional concepts aimed at the production of acoustically bright and dark zones, all the control methods used require a tradeoff between radiation directivity and reproduction accuracy in the bright zone. An alternative solution is proposed which can achieve better performance based on the measures presented simultaneously by inserting a low priority zone named as the "gray" zone. This involves the weighted minimization of mean-squared errors in both bright and dark zones together with the gray zone in which the minimization error is given less importance. This results in the production of directional bright zone in which the accuracy of sound reproduction is maintained with less required input power. The results of simulations and experiments are shown to be in excellent agreement.
\end{abstract}




\section{Introduction}

A number of research studies have been aimed at the simultaneous generation of audible and inaudible regions using an array of sound sources whose interference pattern is controlled [1, 2]. The use of multiple sources has been investigated for the purposes of producing 'personal sound' [3]. Choi et al. [4] used the concept of control regions associated with acoustically bright (loud) and dark (quiet) zones and introduced the so-called acoustic brightness and contrast maximization methods. Shin et al. [5] proposed an efficient alternative control method which maximizes the acoustic energy difference between two adjacent acoustic spaces demonstrating the effectiveness using a spherical array of 40 loudspeakers in an anechoic chamber. Other researchers have focused on personal audio systems [6] which ensure audibility for one listener whilst leaving others nearby in a dark zone. Several private sound field generation techniques have been investigated through the practical implementation of personal audio systems [7-9]. Recently, the robustness of a transformed contrast control method with a regularization factor has been analysed using Lagrange multipliers from the constrained optimization problem [10]. The sound field can also be controlled using the least squares minimization method [11] in order to generate directional sound radiation characteristics [12,13]. Chang and Jacobsen [14] introduced a formulation for the reproduction of a sound field having minimum error in the desired bright zone of the sound field while reducing the sound energy in the dark zone.

In this work, various approaches to the control of sound interference in order to generate the bright and dark zones are investigated for the case of a dual layer loudspeaker array. The linear loudspeaker array having two layers of loudspeakers has been designed in order to control the sound radiation of the devices in all possible directions, whilst a single layer array cannot control the sound radiation in the "backward" direction. All the control methods have been simulated using the electroacoustic transfer functions measured in an anechoic chamber. 
Analyses are presented of the performance of the array in terms of the required total input power, directivity of sound radiation and accuracy of sound reproduction in the bright zone.

These three categories have a trade-off relationship which is not easily satisfied at the same time. Furthermore, they are all frequency dependent characteristics. Consequently, this paper tries to represent the required total input power, directivity of sound radiation and accuracy of sound reproduction simultaneously. The required total input power, given by the squared norm of the source strength vector in the frequency domain, is a measure of driving energy required by the control algorithm applied. The radiation directivity of the loudspeaker array is represented by the radiation patterns averaged within 6 frequency bands. Accuracy of sound reproduction in the bright zone has been evaluated from the error between the reproduced signal and the desired signal in terms of the frequency response function and the time domain impulse response.

With alternative selection methods of the tunable parameters, detailed investigations have been carried out of two conventional control methods: mean squared pressure error minimization, also referred to as pressure matching method [15], and energy difference maximization. Minimization of weighted pressure error and energy, which combines aspects of the two control methods above, has also been analyzed. After the investigations of conventional sound interference control methods above, a weighted minimization approach is proposed here which is shown to be successful in producing a solution which can simultaneously achieve good performance in both radiation directivity and reproduction accuracy with high input efficiency by inserting a "low priority zone".

The paper is structured as follows: the definition of the acoustical problem under consideration is given in Section 2 and the detailed description of the dual layer loudspeaker array used for the experiments is reported in Section 3. Extensive investigations of existing sound interference control methods have been conducted in Section 4, 5 and 6. Section 7 
introduces the proposed weighted minimization method.

\section{Problem definition}

The problem of generating acoustically bright and dark zones is schematically described in Fig. 1(a). The acoustically bright zone, depicted as an arc with angle of $\theta_{b}$, has been defined as the region where higher acoustic energy is desired, whereas the acoustically dark zone, depicted as an arc with angle of $\theta_{d}$, is the region where lower acoustic energy is desired.

For a given frequency $\omega$, the sound pressures generated by the array at the $M_{b}$ and $M_{d}$ control points in the bright and dark zones, respectively, located at $\mathbf{x}_{m}^{b}\left(m=1,2, \cdots M_{b}\right)$ and $\mathbf{x}_{m}^{d}\left(m=1,2, \cdots M_{d}\right)$, can be expressed as the vectors, $\mathbf{p}_{b}=\left[\begin{array}{llll}p\left(\mathbf{x}_{1}^{b}\right) & p\left(\mathbf{x}_{2}^{b}\right) & \cdots & p\left(\mathbf{x}_{M_{b}}^{b}\right)\end{array}\right]^{\mathrm{T}}$ and $\mathbf{p}_{d}=\left[\begin{array}{llll}p\left(\mathbf{x}_{1}^{d}\right) & p\left(\mathbf{x}_{2}^{d}\right) & \cdots & p\left(\mathbf{x}_{M_{d}}^{d}\right)\end{array}\right]^{\mathrm{T}}$. These pressure vectors can be related to the source strength vector, $\mathbf{q}=\left[\begin{array}{llll}q\left(\mathbf{y}_{1}\right) & q\left(\mathbf{y}_{2}\right) & \cdots & q\left(\mathbf{y}_{N}\right)\end{array}\right]^{\mathrm{T}}$ by

$$
\mathbf{p}_{b}=\mathbf{Z}_{b} \mathbf{q}, \mathbf{p}_{d}=\mathbf{Z}_{d} \mathbf{q}
$$

where $N$ is the number of sources and the matrices of transfer functions are defined by

$$
\mathbf{Z}_{b}=\left[\begin{array}{ccc}
Z\left(\mathbf{x}_{1}^{b} \mid \mathbf{y}_{1}\right) & \cdots & Z\left(\mathbf{x}^{b} \mid \mathbf{y}_{N}\right) \\
\vdots & \ddots & \vdots \\
Z\left(\mathbf{x}_{M_{b}}^{b} \mid \mathbf{y}_{1}\right) & \cdots & Z\left(\mathbf{x}_{M_{b}}^{b} \mid \mathbf{y}_{N}\right)
\end{array}\right], \mathbf{Z}_{d}=\left[\begin{array}{ccc}
Z\left(\mathbf{x}_{1}^{d} \mid \mathbf{y}_{1}\right) & \cdots & Z\left(\mathbf{x}_{1}^{d} \mid \mathbf{y}_{N}\right) \\
\vdots & \ddots & \vdots \\
Z\left(\mathbf{x}_{M_{d}}^{d} \mid \mathbf{y}_{1}\right) & \cdots & Z\left(\mathbf{x}_{M_{d}}^{d} \mid \mathbf{y}_{N}\right)
\end{array}\right],
$$

where $Z\left(\mathbf{x}_{m} \mid \mathbf{y}_{n}\right)$ is the acoustic transfer function from the source at $\mathbf{y}_{n}$ to the control point at $\mathbf{x}_{m}$. Eq. (1) can be rewritten in the single form given by

$$
\mathbf{p}=\mathbf{Z q},
$$

where $\mathbf{p}=\left[\begin{array}{ll}\mathbf{p}_{b}{ }^{\mathrm{T}} & \mathbf{p}_{d}{ }^{\mathrm{T}}\end{array}\right]^{\mathrm{T}}$ and $\mathbf{Z}=\left[\begin{array}{ll}\mathbf{Z}_{b}{ }^{\mathrm{T}} & \mathbf{Z}_{d}{ }^{\mathrm{T}}\end{array}\right]^{\mathrm{T}}$. 
All the physical parameters above such as $p, q$ and $Z$ are assumed to have a time dependence of $e^{j \omega t}$. The simulations and experiments described in this paper make use of digital filters to ensure an appropriate input signal is input to each loudspeaker in the array [16]. In this context therefore, the elements of vectors $\mathbf{q}$ and $\mathbf{p}$ can be considered as complex variables in the discrete time domain whilst the elements of the matrix $\mathbf{Z}$ can be interpreted as the z-domain transfer functions relating the discrete time input signals of loudspeakers and output signals of microphones. The values of the elements of $\mathbf{q}$ defining the z-transform of the digital filters are then used to operate on inputs to the array.

\section{Dual layer loudspeaker array}

\subsection{Design of the prototype array}

The dual layer loudspeaker array has been built to enable the evaluation of the performance of several control algorithms when they are practically implemented. The loudspeaker driver, B1S (1" metal cone, moving coil type, 2 watt maximum power) of HiVi Inc. [17], which has an extended low-frequency range when compared to similar size models, has been selected as the individual loudspeaker implemented in the array shown in Fig. 2 (a). The external diameter of the selected individual driver is $3.6 \mathrm{~cm}$. By leaving a small gap of $2 \mathrm{~mm}$ between two adjacent loudspeaker drivers, a distance of $3.8 \mathrm{~cm}$ was then chosen as the inter-element spacing. This spacing would yield a spatial aliasing frequency around $9 \mathrm{kHz}$ for a plane wave propagating to the front of the array [18].

The enclosure is composed of two rectangular parallelepiped wooden cabinets, in which we define the front panel as the face which has the loudspeaker holes, and the back panel as the opposite face. There are eight circular holes on the front panel, spaced $3.8 \mathrm{~cm}$ from each other, and all drivers in each cabinet share a single cavity with the internal dimensions of $4 \times$ 
$3.6 \times 30.5 \mathrm{~cm}$. Fig. 2 (b) shows the completed prototype dual layer array consisting of 16 loudspeakers. The size of the array module, locations of individual loudspeakers and directions, $0^{\circ}$ (on-axis) and $60^{\circ}$ of the array are represented in the same figure. The total width, depth and height of the loudspeaker array are respectively 33, 12 and $6 \mathrm{~cm}$. Based on the frequency response data sheet provided by the manufacturer [17], the specific low frequency limit is $100 \mathrm{~Hz}$ and the high frequency limit is $20 \mathrm{kHz}$. Considering the spatial aliasing frequency of approximately $9000 \mathrm{~Hz}$ defined by the spacing above, this array can in principle be used between 100 and $9000 \mathrm{~Hz}$ for generating a private sound field.

\subsection{Transfer function measurements}

The transfer functions of the designed loudspeaker array have been measured to enable accurate evaluations of the performance of the various candidate control algorithms. The measured transfer functions are considered in the processes of designing a desired signal and computing the optimum source strength vector. Since the measured transfer functions have been obtained by measuring the white noise signal input to each loudspeaker and the radiated pressures at the microphone, they automatically include the acoustical characteristics such as reflections and scattering effects due to the array cabinet. Thus the simulation results using the measured transfer functions are more realistic than those computed using a simple analytical model assuming monopole sources radiating in free field without consideration of the array cabinet.

The measurement system consists of the loudspeaker array mounted on the turntable (B\&K 3922) and one microphone (B\&K 4189) located at the front when the turntable angle is $0^{\circ}$. The distance between the centre of the array and the microphone was set as $3 \mathrm{~m}$ in order to avoid the possible near-field effect within the audio frequency range [19]. As shown in Fig. 2 (c), the measurement system was installed in the ISVR Large Anechoic Chamber $(9.15 \times$ 
$9.15 \times 7.32 \mathrm{~m}^{3}$ ) which allows for an excellent approximation of free field conditions above $80 \mathrm{~Hz}[20]$.

The signals processed by the computer were connected to D/A (Digital to Analog) converters (RME ADI-8 DS) to the digital interface (RME ADI-648). The loudspeaker signals amplified by a custom-made multichannel power amplifier were fed into each loudspeaker in the array. The microphone signal amplified by NEXUS signal conditioner was sent to A/D (Analog to Digital) converter (RME ADI-8 DS). The converter was connected to the same digital interface which was in turn connected to the computer. The measurements were carried out with a sampling frequency of $48000 \mathrm{~Hz}$.

Transfer functions between loudspeakers in the array and the microphone have been measured at every $5^{\circ}$ from $0^{\circ}$ to $355^{\circ}$ by using the turntable, as indicated in Fig. 1 (b).

\subsection{Desired signals}

As will be discussed later, the various methods used to compute the loudspeaker digital filters require the definition of the target or desired signals, which are a set a complex values representing the sound pressure that the array is required to generate at all control points. A simple approach would suggest that the desired signals in the bright zone should be an ideal impulse with a flat frequency response whilst the signals in the dark zone would be set to zero. However, due to the limitation of the loudspeaker array, it is important to design a practically achievable desired signal in the bright zone since the specification of an unrealistic desired signal can lead to impractical or unrealizable control inputs that in turn ensure the experimental results never approach those simulated. As shown in Fig. 3, the amplitude of the reference desired signal in the bright zone, $\hat{p}_{b}$, has been designed to approximately match the measured pressure response of the loudspeaker array. This was 
measured at the on-axis $\left(0^{\circ}\right)$ microphone position with $3 \mathrm{~m}$ distance when the front 8 loudspeakers were driven with the same signal. The high frequency limit defined was based on the spatial aliasing frequency of the proposed dual layer loudspeaker array. The desired signal $\hat{p}_{b}$ was generated by the impulse response of a second order high-pass Butterworth filter in series with an eighth order low-pass Butterworth filter, the two filters having cutoff frequencies of $400 \mathrm{~Hz}$ and $8100 \mathrm{~Hz}$, respectively. The phase of the frequency response function and the time domain impulse response of the desired signal are also reported in Fig. 3.

\section{Minimization of error in mean squared pressure}

The first control algorithm studied in this paper is a frequency-domain least squares approach [11] that minimizes the error between the desired and the reproduced pressures at the control points. The cost function to be minimized has been defined as the sum of a reproduction error term, given by the norm of the difference between the desired and the reproduced pressures, and of an effort penalty term, given by the squared norm of the loudspeaker input source strength vector, weighted by a positive real regularization parameter, set as Eq. (3).

$$
J_{L S}(\mathbf{q})=\|\hat{\mathbf{p}}-\mathbf{p}\|^{2}+\beta\|\mathbf{q}\|^{2}=\|\hat{\mathbf{p}}-\mathbf{Z} \mathbf{q}\|^{2}+\beta\|\mathbf{q}\|^{2}
$$

where $\beta$ is the Tikhonov regularization parameter and $\hat{\mathbf{p}}$ is the vector of desired pressure signals. The desired pressure in the bright zone is given by the signal $\hat{p}_{b}$ introduced above and represented in Fig. 3, whilst the target pressure in the dark zone is set to zero. The desired pressure vector is given by $\hat{\mathbf{p}}=\left[\begin{array}{ll}\hat{\mathbf{p}}_{b}^{\mathrm{T}} & \mathbf{0}_{M_{d}}{ }^{\mathrm{T}}\end{array}\right]^{\mathrm{T}}$ where $\hat{\mathbf{p}}_{b}$ is the $M_{b}$ by 1 vector with identical elements $\hat{p}_{b}$ and $\mathbf{0}_{M_{d}}$ is the $M_{d}$ by 1 vector with all zero elements. 
The source strength vector that minimizes the cost function, $J_{L S}$ is obtained from [11]

$$
\mathbf{q}_{L S}=\left[\mathbf{Z}^{\mathrm{H}} \mathbf{Z}+\beta \mathbf{I}\right]^{-1} \mathbf{Z}^{\mathrm{H}} \hat{\mathbf{p}}
$$

Based on the concept of the cost function represented in Eq. (3), this method minimizes the differences between the desired and reproduced pressures.

The controllable parameter is the Tikhonov regularization parameter, $\beta$ which makes possible the inversion of the very often ill-posed matrix $\mathbf{Z}^{\mathrm{H}} \mathbf{Z}$ in Eq. (4). Based on the cost function in Eq. (3), it can also directly control the amount of the input power required given by $\|\mathbf{q}\|^{2}$. In addition, it is the indirect parameter to control the trade-off between energy difference in the bright and dark zones and required input power.

\subsection{Selection of $\beta$}

The parameter $\beta$ can be varied from zero to infinity, and the corresponding cost function gradually changes from containing only the reproduction error term to containing only the effort penalty term; therefore, $\beta$ can be used to control the power output from the loudspeakers at the expense of a higher performance error. This concept is clearly illustrated by the so-called L-curve [21]. In view of this consideration, $\beta$ can be chosen using a constrained minimization approach, wherein the performance error is minimized with the constraint that the total input power must not exceed $5 \mathrm{~dB}$ relative to unity voltage input to the loudspeakers, which is around half of the required total input power in the case where $\beta$ is conventionally chosen by using Eq. (5) below. The data used for the determination of $\beta$ are reported in Fig. 4, wherein the total input power is plotted as a function of $\beta$. The parameter $\beta$ has thus been fixed when the curve of total input power of the loudspeaker array reaches 
the value of 5dB. Fig. 4 also shows the sound pressure level difference between the bright and dark zones on a dB scale. As $\beta$ increases, the required total input power deceases while the sound pressure level difference decreases, regardless of frequency. It is because of the cost function represented in Eq. (3) implies that an increased value of $\beta$ imposes more control effort on the minimization of the required inputs than the minimization of the error between desired and reproduced signals.

Gauthier et al. [22] used the concept of normalized Tikhonov regularization which controls the smallest singular value relative to the largest one. Similarly, the regularization parameter can be also chosen to be proportional to the squared norm of the transfer function matrix (that is its first and largest singular value), such that

$$
\beta=\beta_{0}\|\mathbf{Z}\|^{2}=\beta_{0} \sigma_{1}^{2}
$$

where $\sigma_{1} \quad$ is the largest singular value of the transfer function matrix $\mathbf{Z}$, and the parameter $\beta_{0}$ is a real positive constant number. As shown in the Appendix, the parameter $\beta_{0}$ can be chosen to adjust the upper bound of the condition number of the matrix to be inverted whilst ensuring sufficient headroom for the required input power.

The frequency dependent values of $\beta$ calculated using Eq. (5) with $\beta_{0}=10^{-2}$ sets the upper bound of the condition number as 100 (actually 101, see Eq. (A.5)) and gives $\sim 20 \mathrm{~dB}$ headroom (see Eq. (A.10)) for the required input power. The values selected by the procedure introduced above are presented in Fig. 5 (a), while Fig. 5 (b) represents the resultant total input powers associated with the range of these two criteria for the choice of $\beta$. Based on the observation of Fig.4 and 5, the selected $\beta$ increases in the low frequency range below $2000 \mathrm{~Hz}$. However, there is no clear trend in the value of $\beta$ when the frequency is higher than $2000 \mathrm{~Hz}$ due to the conditioning of the system matrix composed of the 
measured transfer functions.

Note that the curves in Fig. 4 for the decision procedure vary with changes of the transfer function matrix composed of relationship between the control points on the control circle and the loudspeakers built in the array.

\subsection{Simulations}

As observed in the cost function of Eq. (3), the minimization method introduced tries to match the pressure magnitudes and phases at all the control points by controlling the strengths of the source input signals. The control points in the bright zone $\left(\theta_{b}=40^{\circ}\right)$ are represented with circles and those in the dark zone $\left(\theta_{d}=320^{\circ}\right)$ are depicted with crosses on the control circle with $R=3 \mathrm{~m}$ as shown in Fig. 1 (b). The source strength vector $\mathbf{q}_{L S}$ was computed with Eq. (4) to the half of the sampling frequency of $48 \mathrm{kHz}$ and the elements of $\mathbf{q}_{L S}$ representing loudspeaker filters has been convolved with white noise with the same sampling frequency in order to obtain the input signals for the given loudspeakers [11]. This procedure makes possible the broadband performance evaluation of radiation directivity and reproduction accuracy. Simulations have been undertaken with the measured transfer functions introduced in Section 3.2, which means that the elements of the matrix in Eq. (2) are the electro-acoustical transfer functions between the loudspeaker input signals and the sound pressure signals measured at the control points. The radiation directivity of the method presented has been represented in Fig. 6 for the two selection criteria for the choice of $\beta$. The radiation patterns of the loudspeaker array have been averaged within 6 frequency bands defined by the ranges $88 \sim 177 \mathrm{~Hz}, 177 \sim 355 \mathrm{~Hz}, 355 \sim 710 \mathrm{~Hz}, 710 \sim 1420 \mathrm{~Hz}, 1420 \sim$ $2840 \mathrm{~Hz}$ and $2840 \sim 8000 \mathrm{~Hz}$. The first 5 bands are defined by the octave frequency bands and the last band includes higher frequency components up to $8000 \mathrm{~Hz}$. Fig. 7 represents the 
frequency responses (amplitude and phase) and the impulse responses of the array at the three control points 1, 3 and 5 within the bright zone, as illustrated in Fig. 1(b). This figure also compares the desired signal with the presented minimization control results using the two methods of selection of $\beta$.

Based on Fig. 6 and 7, the radiation directivity and the reproduction accuracy of the minimization methods with both values of $\beta$ are similar to each other. With respect to the changes of $\beta$ shown in Fig. 5, the radiation patterns of the two cases are slightly different from each other in the third and fifth frequency bands as shown in Fig. 6. The directivity of sound radiation with the selected $\beta$ shows smaller side lobes in the third frequency band and greater rear lobes in the fifth frequency band. The accuracy of the sound reproduction in Fig. 7 shows more than $5 \mathrm{~dB}$ error in the reproduced sound relative to the desired signal below $1000 \mathrm{~Hz}$ at the first control point. In addition, the reproduction accuracy has been degraded as the observed location moves further away from the first control point.

\section{Maximization of energy difference}

The second control algorithm analyzed in this work is the energy difference maximization method [5]. The cost function to be maximized is given by Eq. (6) which quantifies the energy difference between two acoustic zones, divided by the total input power. This is given by

$$
J_{E D}(\mathbf{q})=\frac{e_{b}-\alpha e_{d}}{e_{q}}=\frac{\mathbf{q}^{\mathrm{H}}\left(\mathbf{R}_{b}-\alpha \mathbf{R}_{d}\right) \mathbf{q}}{\mathbf{q}^{\mathrm{H}} \mathbf{q}},
$$

where $e_{b}=\mathbf{q}^{\mathrm{H}} \mathbf{R}_{b} \mathbf{q}, \quad e_{d}=\mathbf{q}^{\mathrm{H}} \mathbf{R}_{d} \mathbf{q}$ and $e_{q}=\mathbf{q}^{\mathrm{H}} \mathbf{q}$. The matrices $\mathbf{R}_{b}$ and $\mathbf{R}_{d}$ are the spatially averaged correlation matrices composed of the elements in the transfer function matrix $\mathbf{Z}_{b}$ and $\mathbf{Z}_{d}$. These matrices are defined as $\mathbf{R}_{b}=\mathbf{Z}_{b}{ }^{\mathrm{H}} \mathbf{Z}_{b} / M_{b}$ and $\mathbf{R}_{d}=\mathbf{Z}_{d}{ }^{\mathrm{H}} \mathbf{Z}_{d} / M_{d}$ 
with $M_{b}, M_{d}$ control points in the bright and dark zones. The optimized source strength vector $\mathbf{q}_{E D}$ that maximizes the cost function in Equation (6) can be calculated as the eigenvector associated with the largest eigenvalue of the following eigenvalue problem:

$$
J_{E D}\left(\mathbf{q}_{E D}\right) \mathbf{q}_{E D}=\left(\mathbf{R}_{b}-\alpha \mathbf{R}_{d}\right) \mathbf{q}_{E D} .
$$

Note that matrix $\left(\mathbf{R}_{b}-\alpha \mathbf{R}_{d}\right)$ is Hermitian; therefore its eigenvalues are real. There are several advantages of using this maximization method. Firstly, the weighting factor $\alpha$, which is a positive real number, can be used to tune the amount of control effort for the energy in the second zone. Secondly, no matrix inversion is required to find the optimum solution and thus the associated conditioning problems are avoided. Furthermore, it should be noted that if $\mathbf{q}_{E D}$ is an eigenvector that satisfies the relation of Eq. (7), then the same relation is also satisfied by the vector $\zeta \mathbf{q}_{E D}$, wherein $\zeta$ is an arbitrary complex number. In the discussion reported below of this control method, the norm of the source strength vector was chosen to be unitary. However, this method only maximizes the energies between two acoustic zones without consideration of the phase of the reproduced sound pressure, so that the accuracy of sound reproduction in the bright zone cannot be guaranteed. This suggests that any method dealing with energy-related terms cannot guarantee more accurate reproduction of the original sound than the method minimizing the error between desired and reproduced signals. To overcome this problem, the phase compensated source strength vector, $\mathbf{q}_{c}$ has been defined by

$$
\mathbf{q}_{c}=F_{p} \cdot \mathbf{q}_{E D}
$$

with an all-pass phase correction filter, $F_{p}=\left(\hat{p}_{b} /\left|\hat{p}_{b}\right|\right) \cdot\left(\left|\mathbf{Z}_{b}^{k} \mathbf{q}_{E D}\right| / \mathbf{Z}_{b}^{k} \mathbf{q}_{E D}\right)$. The phases of all source strengths have been modified in order to achieve a signal with the desired phase at the $k$-th control point in the bright zone. In this paper, the control point at the on-axis position 
corresponding to position 1 in Fig. 1(b) has been selected for phase compensation.

\subsection{Selection of $\alpha$}

One of the advantages of the energy difference control method is that the tuning factor $\alpha$ enables the adjustment of the relative importance between acoustic energy difference between the two control zones and the efficiency of radiation of the sources into the bright zone. The choice of $\alpha$ can greatly affect the performance of the array so that it is important to have reasonable procedure for selecting $\alpha$.

Fig. 8 shows the averaged sound pressure level in the bright zone and the sound pressure level difference between bright and dark zones as a function of $\alpha$, for different operating frequencies. The ideal objective is to maximize the energy difference between bright and dark zones maintaining the sound pressure in the bright zone constant at a relatively high level. However, the plotted data show that the value of $\alpha$ that maximizes the energy difference corresponds to very low level of sound pressure in the bright zone. In the light of this analysis, $\alpha_{q}$ has been chosen as the value of $\alpha$ that maximizes the energy difference with the constraint that the sound pressure in the bright zone should not fall below $20 \mathrm{~dB}$ if possible. This value has been decided based on the averaged signal level obtained by turning on 8 loudspeakers on the front layer of array as shown in Fig. 3. Conversely, the value of $\alpha_{c}$ has been selected as the value of $\alpha$ corresponding to the maximum contrast between pressures in the bright and dark zones. The range of $\alpha, 1$ to 300 , is dependent on the geometry of array and the arrangements of the loudspeakers and control points. This is relevant because

all curves start to converge as represented in Fig. 8. Note that the curves in Fig. 8 for the decision procedure vary with changes of numbers or locations of the control points on the control circle as well as the number and disposition of loudspeakers in the array. 
Fig. 9 represents the selected frequency dependent tuning factors, $\alpha_{q}$ and $\alpha_{c}$ based on the procedure illustrated in Fig. 8 and the corresponding total input power curves, which are perfectly overlaid.

\subsection{Simulations}

The maximization method introduced here controls the energy magnitudes at all the control points without considering the phase components as represented in Eq. (6) even though the phase compensation filter in Eq. (8) based on only one control point has been inserted. In addition, the cost function does not include the desired signal term so that there is no guarantee of the close replication of the desired signal.

The simulation results produced by the method presented in this section have been obtained with the same simulation arrangements used in Section 4.2. The radiation directivity of the maximization method with $\alpha_{q}$ and $\alpha_{c}$ has been represented in Fig. 10 by the radiation patterns of the loudspeaker array. These have been averaged within the same 6 frequency bands introduced before. The accuracy of sound reproduction has been described in Fig. 11 by the frequency response function and the impulse responses at three control points.

Fig. 10 and 11 demonstrate that the energy difference control method can result in different performance in terms of radiation directivity and reproduction accuracy depending on the method for choosing the tuning factor $\alpha$. The simulated results with the tuning factor $\alpha_{q}$ show inferior directivity performance than those with the contrast promoting tuning factor $\alpha_{c}$. However, in terms of the reproduction accuracy in the bright zone, results with $\alpha_{q}$ exhibit closer to the desired signal than those with $\alpha_{c}$, which loses low frequency energy below $2000 \mathrm{kHz}$. Of course, this effect may be compensated by equalizing the source strength 
and obtain a flat frequency response at the reference control point. This would, however, result in a large low-frequency boost which may lead to instability. As for the impulse responses shown in Fig. 11 ( $\mathrm{g}, \mathrm{h}$ and $\mathrm{i}$ ), it is possible to observe impulse responses closer to the desired signal near the control point 1 because the phase has been corrected by the allpass filter in Eq. (8).

\section{Minimization of weighted pressure error and energy}

As mentioned in Section 5, the energy difference maximization control method can directly control the directivity of sound radiation using the tuning factor, $\alpha$. However, the method does not allow direct control of the phase and amplitude of the reproduced pressure at the control points. In order to get a more refined solution, a method has been proposed that combines the advantages of both methods above [14]. This method relies on a cost function that includes the reproduction error in the bright zone and the energy in the dark zone and which can be represented as

$$
J_{W P E}(\mathbf{q})=(1-\kappa)\left\|\hat{\mathbf{p}}_{b}-\mathbf{p}_{b}\right\|^{2}+\kappa\left\|\mathbf{p}_{d}\right\|^{2}+\beta\|\mathbf{q}\|^{2}
$$

By equating to zero the partial derivatives with respect to $\mathbf{q}$, the optimal solution is obtained as

$$
\mathbf{q}_{W P E}=\left(\kappa \mathbf{Z}_{d}^{\mathrm{H}} \mathbf{Z}_{d}+(1-\kappa) \mathbf{Z}_{b}^{\mathrm{H}} \mathbf{Z}_{b}+\beta \mathbf{I}\right)^{-1}(1-\kappa) \mathbf{Z}_{b}^{\mathrm{H}} \hat{\mathbf{p}}_{b},
$$

where the parameter, $\kappa(0 \leq \kappa \leq 1)$ adjusts the control weights associated with the minimization respectively of the error and dark zone potential energy while $\beta$ is the regularization factor associated with the required input energy.

The tuning parameter $\kappa$ is conceptually similar to the tuning factor $\alpha$ of the energy difference maximization method. In fact, this method may be simply interpreted as a weighted minimization method of error in mean squared pressure, wherein the cost function includes different weights for the reproduction errors in the bright zone and in the dark zone, 
respectively.

\subsection{Simulations}

The simulated control results using the weighted pressure error and energy minimization method have been represented with two different values of $\kappa$ which are 0.5 and 0.9 . When the parameter $\kappa$ is 0.5 , the cost function in Eq. (9) is conceptually equal to that of minimization of error in mean squared pressure shown in Eq. (3). The control with $\kappa=0.9$ is the representative case with relatively large weighting on the minimization of pressure in the dark zone rather than the error minimization in the bright zone. Fig. 12 shows the obtained total input energy required with $\beta$ computed using Eq. (5) when $\beta_{0}=10^{-2}$. The directivity of sound radiation has been represented in Fig. 13 by the radiation patterns of the loudspeaker array which have been averaged within the 6 frequency bands. The accuracy of sound reproduction is also described in Fig. 14 with the frequency response functions and the impulse responses at the three control points in Fig. 1(b).

Based on the simulation results in both Fig. 13 and 14, it is clear that control with larger $\kappa$ gives better directivity performance with less amount of side lobes whilst it can lose the accuracy of reproduced sound in the bright zone. With the smaller value of $\kappa$, the control results represent the reproduced sound closer to the desired signal and worse directivity with more side lobes. The accuracy of sound reproduction is not satisfactory for both cases due to the low frequency discrepancy from the desired signal especially below $1000 \mathrm{~Hz}$. In addition, it cannot guarantee the reproduction accuracy within all of the bright zone area. For control points away from the on-axis position, the accuracy of sound reproduction deteriorates for both values of $\kappa$. Once again, the results show that the array efficiency at low frequency can be increased at the expenses of the directivity performance, and vice versa. 


\section{Weighted pressure matching with a low priority zone}

This section introduces a control method called weighted pressure matching with a low priority zone. With the conventional acoustical problem definition shown in Fig. 1 having only bright and dark zones, it is difficult to achieve good radiation directivity and reproduction accuracy with high efficiency of required input power due to the trade-offs in these parameters. Consequently, instead of suffering the trade-off in performance resulting from a conventional approach, these three performance measures can be simultaneously enhanced by inserting a low priority zone where users have less concerns about the control of sound. The underlying practical assumption is that the system users desire a listening area characterized by accurate sound reproduction and a quiet area at the same time, whilst the low priority zone corresponds to a region without listener or with listeners who don't care about noise level. Fig. 15 describes the modified acoustical problem after insertion of the low priority zone, called the 'gray zone'. In other words, the control points belong now to three acoustic zones, called the 'bright', 'gray' and 'dark' zones, which are respectively described by four arcs with angles of $\theta_{b}, \theta_{g}$ and $\theta_{d}$ on the control circle.

Apart from the parameters defined in Section 2, the values of the sound pressures at the $M_{g}$ discrete points in the gray zone, located at $\mathbf{x}_{m}^{g}\left(m=1,2, \cdots M_{g}\right)$, are represented by the vector $\mathbf{p}_{g}=\left[\begin{array}{llll}p\left(\mathbf{x}_{1}^{g}\right) & p\left(\mathbf{x}_{2}^{g}\right) & \cdots & p\left(\mathbf{x}_{M_{g}}^{g}\right)\end{array}\right]^{\mathrm{T}}$. The relation between this pressure vector and the source strength vector is given by

$$
\mathbf{p}_{g}=\mathbf{Z}_{g} \mathbf{q},
$$

where the matrix of transfer functions is defined by 


$$
\mathbf{Z}_{g}=\left[\begin{array}{ccc}
Z\left(\mathbf{x}_{1}^{g} \mid \mathbf{y}_{1}\right) & \cdots & Z\left(\mathbf{x}^{g}{ }_{\mid} \mathbf{y}_{N}\right) \\
\vdots & \ddots & \vdots \\
Z\left(\mathbf{x}_{M_{g}}^{g} \mid \mathbf{y}_{1}\right) & \cdots & Z\left(\mathbf{x}_{M_{g}}^{g} \mid \mathbf{y}_{N}\right)
\end{array}\right] .
$$

Eq. (1) and (11) can be also rewritten as a single equation as

$$
\mathbf{p}=\mathbf{Z q}
$$

where $\mathbf{p}=\left[\begin{array}{lll}\mathbf{p}_{b}{ }^{\mathrm{T}} & \mathbf{p}_{g}{ }^{\mathrm{T}} & \mathbf{p}_{d}{ }^{\mathrm{T}}\end{array}\right]^{\mathrm{T}}$ and $\mathbf{Z}=\left[\begin{array}{lll}\mathbf{Z}_{b}{ }^{\mathrm{T}} & \mathbf{Z}_{g}{ }^{\mathrm{T}} & \mathbf{Z}_{d}{ }^{\mathrm{T}}\end{array}\right]^{\mathrm{T}}$.

The cost function for the acoustical problem proposed in Fig. 15 can be represented by the weighted minimization forms given by

$$
J(\mathbf{q})=\left\|\hat{\mathbf{p}}_{b}-\mathbf{p}_{b}\right\|^{2}+\gamma\left\|\mathbf{p}_{g}\right\|^{2}+\left\|\mathbf{p}_{d}\right\|^{2}+\beta\|\mathbf{q}\|^{2}
$$

where $\gamma(0 \leq \gamma \leq 1)$ is the weighting parameter which controls the amount of importance for the gray zone. The cost function in Eq. (13) can be rewritten in matrix form as,

$$
J_{W P M}(\mathbf{q})=\|\boldsymbol{\Gamma}(\hat{\mathbf{p}}-\mathbf{p})\|^{2}+\beta\|\mathbf{q}\|^{2}
$$

where $\hat{\mathbf{p}}=\left[\begin{array}{ll}\hat{\mathbf{p}}_{b}^{\mathrm{T}} & \mathbf{0}_{\left(M_{g}+M_{d}\right)}^{\mathrm{T}}\end{array}\right]^{\mathrm{T}}$ and $\boldsymbol{\Gamma}^{2}=\operatorname{diag}\left(\left[\begin{array}{lll}\mathbf{1}_{M_{b}}^{\mathrm{T}} & \gamma \mathbf{1}_{M_{g}}^{\mathrm{T}} & \mathbf{1}_{M_{d}}^{\mathrm{T}}\end{array}\right]\right)$. Here $\mathbf{1}_{m}^{\mathrm{T}}$ is the $M_{d}$ by 1 vector fully populated with unity elements. Note that, in principle, it is possible to apply a different weight to the reproduction error associated with each control point.

By executing the partial derivation with respect to $\mathbf{q}$ and setting it to zero the optimum solution can be obtained as below,

$$
\mathbf{q}_{W P M}=\left(\mathbf{Z}^{\mathrm{H}} \boldsymbol{\Gamma}^{2} \mathbf{Z}+\beta \mathbf{I}\right)^{-1} \mathbf{Z}^{\mathrm{H}} \boldsymbol{\Gamma}^{2} \hat{\mathbf{p}}
$$

It can be easily seen that the formulations in Eq. (15) can express the cost functions in Eq. (4)

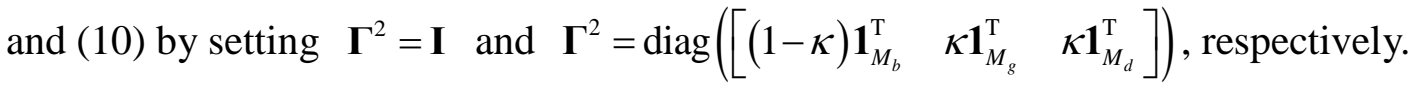

\subsection{Simulations}


Obviously, the control results of weighted minimization introduced are dependent on the arrangements of control points and on the values of $\gamma$. In this section, simulations are presented with two different arrangements of control points with two different values of $\gamma$. Fig. 16 describes the two types of control point arrangements. The first control point arrangement in Fig. 16 (a) has the same $\theta_{b}$ and $\theta_{d}$ as $40^{\circ}$ to the front and back of the loudspeaker array along with a gray zone in the rest of the control circle while the second arrangement of control points has bright $\left(\theta_{b}=40^{\circ}\right)$ and dark $\left(\theta_{d}=170^{\circ}\right)$ zones as shown in Fig. 16 (b). The two values of the weighting parameter $\gamma(1 / 2$ and 1/16) were selected as representative of comparatively high and low values in order to show the effect of changing the control priority for the gray zone. The regularization factor $\beta$ has been calculated using Eq. (5) with $\beta_{0}=10^{-2}$. The total input power curves for the control point arrangement described in Fig. 16 have been reported in Fig. 17 for those two different values of $\gamma$

Using the first control point arrangement depicted in Fig. 16 (a), the effects of weighting parameter $\gamma$ can be explained based on the directivity of sound radiation in Fig. 18. The accuracy of sound reproduction in Fig. 19 has been represented by the frequency response function and the time domain impulse responses at the three control points $(1,3$ and 5) with respect to the change of $\gamma$. The control method with $\gamma=1 / 16$, releases more energy to the gray zone so that it generates sound closer to the desired signal in the bright zone and quieter in the dark zone than the control results with $\gamma=1 / 2$.

With the second control point arrangement in Fig. 16 (b), the radiation directivity and reproduction accuracy have been plotted in Fig. 20 and 21, respectively. It is possible to get a wider dark zone with the second control point arrangement than that with the first.

The tendency of the control results with respect to the values of $\gamma$ is similar regardless 
of the control point arrangement. With a smaller value of $\gamma$, the simulation results show better accuracy of sound reproduction in the bright zone and lower sound level in the dark zone. Note that it is possible by radiating more sound to the gray zone. When the value of $\gamma$ is larger, inferior accuracy of sound reproduction in the bright zone with higher sound level in the dark zone results due to the stronger restriction of sound radiation to the gray zone.

Insertion of the gray zone allows relatively accurate sound reproduction at the control points within the bright zone, as shown in Fig. 19 and 21, and at the same time, lower sound pressure is generated in the dark zone as observed in Fig. 18 and 20 than the conventional methods introduced in the previous sections. The total input powers exhibits small variations from -5 to $10 \mathrm{~dB}$ within controlled frequency range as represented in Fig. 17.

\subsection{Experiments}

All the simulations in this paper that are based on the measured transfer functions are expected to be well-matched with the experiments. In this section, one of the simulated results, controlled by the weighted minimization with a low priority zone (the second weighting function, $\gamma=1 / 16)$, has been verified with the experimental result obtained with the same experimental setup used for obtaining the transfer functions in Fig. 2. For the experiment, the input signal (broad band white noise) was convolved with the 16 filters that determine the required input to each loudspeaker. These filters are obtained by calculating the optimum solution of Eq. (15) with respect to the frequencies ranging from $100 \mathrm{~Hz}$, the low frequency limit of individual loudspeaker, to $9 \mathrm{kHz}$, the spatial aliasing frequency of the array.

Fig. 22 shows the overlaid radiation patterns of simulations and experiments. Control of the weighted minimization with a low priority zone has been applied when $\gamma=1 / 16$ with control point arrangement in Fig. 16 (b). The frequency band averaged radiation patterns of 
the experiments show good matches with the simulated results. The reproduction accuracy measures in Fig. 23, frequency response functions along with time domain impulse responses, also show well matched results between simulations and experiments.

Based on the results in Fig. 22 and 23, precise measures of transfer functions and carefully designed filters, which do not generate unrealistic loudspeaker signals, can guarantee minimal mismatches between simulations and experiments. For the same reason, it is confidently expected that all the simulations shown in this paper are well-matched with the experiments.

\section{Comparisons}

In order to evaluate the methods investigated in this paper, several comparisons are presented here of the six methods consisting of the following: minimization of error in mean squared pressure with the value of $\beta\left(Q_{L M}-\beta_{i}\right)$ selected by the proposed procedure, maximization of energy difference with both $\alpha_{c}$ and $\alpha_{q}\left(Q_{E D^{-}} \alpha_{c}, Q_{E D^{-}} \alpha_{q}\right)$, minimization of weighted pressure error and energy with $\kappa=0.9\left(Q_{W P E}(\kappa=0.9)\right)$ and weighted minimization with a low priority zone $(\gamma=1 / 16)$ with the control point arrangement illustrated in Fig. 16 (a) $\left(Q_{W P M^{-}} W_{1}(\gamma=1 / 16)\right)$ and Fig. $16(\mathrm{~b})\left(Q_{W P M^{-}} W_{2}(\gamma=1 / 16)\right)$.

Firstly, the resultant energy contrast between bright and dark zones has been calculated from

$$
\text { Contrast }=10 \log _{10}\left(\frac{M_{d}}{M_{b}} \frac{\left\|\mathbf{p}_{b}\right\|^{2}}{\left\|\mathbf{p}_{d}\right\|^{2}}\right) \text {. }
$$

Fig. 24 represents the comparison of the averaged contrast of the six methods within the same six frequency bands introduced in Fig. 6. It shows that the energy difference maximization method with contrast promoted $\alpha_{q}\left(Q_{E D^{-}} \alpha_{q}\right)$ shows the best contrast performance when the 
control points are defined as Fig. 1 (b), whilst the weighted minimization with a low priority zone $\left(Q_{W P M^{-}}-W_{1}(\gamma=1 / 16)\right)$ shows the best contrast within the 2, 3 and 4 frequency bands when the control points are arranged as in Fig. 16 (a). Secondly, in order to provide an indication of reproduction accuracy, an evaluation is presented of the averaged mismatches between the frequency response functions of the desired and reproduced signals at control points in the bright zone. This is calculated from

$$
\mathrm{FRF}_{\text {error }}=10 \log _{10}\left(\frac{1}{M_{b}}\left\|\hat{\mathbf{p}}_{b}-\mathbf{p}_{b}\right\|^{2}\right)
$$

As shown in Fig. 25, both $Q_{W P M}$ methods result in the minimum FRF error, which means the best match with the desired signal, whereas $Q_{E D^{-}} \alpha_{c}$ has the largest FRF error among the methods compared within almost all frequency bands. Furthermore, within the six frequency bands, the averaged efficiency of the loudspeaker array defined as the ratio of the averaged squared pressure radiated in the bright zone to the total input power is given by

$$
\text { Efficiency }=10 \log _{10}\left(\frac{1}{M_{b}} \frac{\left\|\mathbf{p}_{b}\right\|^{2}}{\|\mathbf{q}\|^{2}}\right)
$$

The results are shown in Fig. 26. The lowest efficiency has been shown in case of $Q_{E D^{-}} \alpha_{C}$ whilst the most efficient method is $Q_{E D^{-}} \alpha_{q}$ which shows that the efficiency of the energy difference maximization method can effectively be adjusted by the selection of the tuning parameter, $\alpha$.

By making use of the low priority zone (the gray zone), $Q_{W P M}$ shows the best sound reproduction in terms of similarity to the desired signal in the bright zone whilst still ensuring reasonably good radiation efficiency of the loudspeaker array. In addition, as shown in Fig. 24 (b), the energy contrast between bright and dark zones is comparatively good when the control points are arranged in the layout of Fig. 16 (a). Based on these comparison results, the method employing the gray zone shows the best compromises between energy contrast, 
radiation efficiency and accuracy of sound reproduction. The other methods are less flexible in achieving the balance among these three performance measures.

\section{Discussions and Conclusions}

A prototype dual layer loudspeaker array with 16 loudspeakers has been designed and constructed in order to practically implement several algorithms for controlling sound interference. All the simulations have been undertaken with the measured transfer functions including the acoustical and electrical characteristics of the real array.

Conventional control methods, minimization error in mean squared pressure (pressure matching) and maximization of energy difference, have been investigated with the proposed parameter selection procedures for the regularization factor $\beta$ and the tuning factor $\alpha$ based on physically meaningful information. In case of minimization method, it turns out to be possible to control the required input power by selecting regularization factor $\beta$. The tuning factor $\alpha$ of the maximization method can be chosen to achieve better contrast between the bright and dark zones or better accuracy of reproduction. In addition, it is also observed that the accuracy of sound reproduction in the bright zone controlled by the maximization method cannot be better than that of minimization control due to the intrinsic limit of the cost function which considers only the energy not the phase.

Control method of minimizing weighted pressure error and energy, conceptually combining both conventional methods, has been investigated in order to achieve the required radiation directivity whilst maintaining the reproduction accuracy within the bright zone. Even though this method has been proposed as a combination of maximization and minimization methods considering both radiation directivity and reproduction accuracy, it still is required to be tuned by those two parameters, $\kappa$ and $\beta$ in the trade-off relationship 
between the two performances.

Whilst the conventional control methods result in a trade-off between the radiation directivity and reproduction accuracy, the proposed method in this paper, consisting of weighed pressure matching with a low priority zone, controls the source strengths in order to obtain the reproduced sound closer to the desired in the bright zone as well as reduced energy in the dark zone. In other words, both performances in the trade-off relationship, accuracy of sound reproduction and energy contrast between bright and dark zones, has been improved by inserting a low priority zone, where there is no concern for acoustical performance, and this is called the gray zone with comparatively low change of the input power. This algorithm can also adjust the priority of control efforts in the gray zone based on the control point arrangement using the $\gamma$ parameter. The results show a wider bright zone, characterized by more accurate sound reproduction, and higher contrast of sound level between the bright and dark zones. Excellent agreement between simulations and experiments has also been shown.

It may be useful to provide users with intuitive tuning parameters to enable the trade-offs between radiation directivity and reproduction accuracy. In this sense, the proposed weighted minimization with a low priority zone can provide an additional tuning factor, by inserting a selectable gray zone, whilst still retaining the capability that includes all of the features of the conventional methods.

\section{Acknowledgements}

This work was partially supported by the Korea Research Foundation funded by the Korean Government [KRF-2008-357-D00198], by the Royal Academy of Engineering (UK) and by the Engineering and Physical Sciences Research Council (UK).

\section{Appendix}


The optimal solution obtained by the least squares method shown in Eq. (4) can be rewritten as Eq. (A.1) below by using the singular value decomposition of the matrix of the acoustic transfer function. Thus

$$
\mathbf{q}_{L S}=\mathbf{V} \mathbf{C}^{-1} \boldsymbol{\Sigma}^{\mathrm{H}} \mathbf{U}^{\mathrm{H}} \hat{\mathbf{p}}
$$

where $\mathbf{U}$ and $\mathbf{V}$ are matrices with orthonormal columns, $\boldsymbol{\Sigma}$ is the diagonal matrix which has nonnegative decreasing diagonal elements called singular values, $\sigma_{n}[22]$, and the matrix $\mathbf{C}$ is defined as

$$
\mathbf{C}=\boldsymbol{\Sigma}^{\mathrm{H}} \boldsymbol{\Sigma}+\beta \mathbf{I}=\left[\begin{array}{ccc}
\sigma_{1}^{2}+\beta & 0 & 0 \\
0 & \ddots & 0 \\
0 & 0 & \sigma_{N}{ }^{2}+\beta
\end{array}\right]
$$

The conditioning of the inversion of the matrix $\mathbf{C}$ can be observed by the condition number of the matrix given by

$$
\operatorname{cond}(\mathbf{C})=\frac{\sigma_{1}^{2}+\beta}{\sigma_{N}^{2}+\beta}
$$

When the smallest singular value $\sigma_{N}$ approaches to zero, the condition number of the matrix $\mathbf{C}$ reaches its largest value. Thus

$$
\operatorname{cond}(\mathbf{C}) \leq \frac{\sigma_{1}^{2}+\beta}{\beta}
$$

Eq. (A.4) can be rewritten by replacing the regularization factor using $\beta=\sigma_{1}^{2} \beta_{0}$ such that

$$
\operatorname{cond}(\mathbf{C}) \leq \frac{1}{\beta_{0}}+1
$$

Based on Eq. (A.5), it is obvious that the upper bound of the condition number of the matrix C to be inverted can be controlled by the parameter $\beta_{0}$. 
Now note that Eq. (4) can also be rewritten as Eq. (A.6) by replacing $\left(\boldsymbol{\Sigma}^{\mathrm{H}} \boldsymbol{\Sigma}+\beta \mathbf{I}\right)^{-1} \boldsymbol{\Sigma}^{\mathrm{H}}$ by $\boldsymbol{\Sigma}^{\#}$ such that

$$
\mathbf{q}_{L S}=\mathbf{V} \Sigma^{\#} \mathbf{U}^{\mathrm{H}} \hat{\mathbf{p}}
$$

The norm of the Eq. (A.6) calculated by the root mean square of all the source strengths can be written in terms of the inequality [23]

$$
\left\|\mathbf{q}_{L S}\right\| \leq\|\mathbf{V}\|\left\|\boldsymbol{\Sigma}^{\#}\right\|\left\|\mathbf{U}^{\mathrm{H}}\right\|\|\hat{\mathbf{p}}\|
$$

It therefore follows, from the orthonormality of the matrices $\mathbf{U}$ and $\mathbf{V}$, that

$$
\left\|\mathbf{q}_{L S}\right\| \leq \max \left(\frac{\sigma_{n}}{\sigma_{n}{ }^{2}+\beta}\right)\|\hat{\mathbf{p}}\|=\frac{1}{2 \sqrt{\beta}}\|\hat{\mathbf{p}}\|
$$

Once the relationship of $\beta=\sigma_{1}^{2} \beta_{0}$ is used in Eq. (A.8), then it follows that

$$
\left\|\mathbf{q}_{L S}\right\| \leq \frac{1}{2 \sigma_{1}} \sqrt{\frac{M_{b}}{\beta_{0}}}
$$

On a logarithmic scale, this can be written as

$$
20 \log _{10}\left\|\mathbf{q}_{L S}\right\| \leq 20 \log _{10} \frac{\sqrt{M_{b}}}{2 \sigma_{1}}-10 \log _{10} \beta_{0}
$$

Consequently, maximum required total input power can be controlled by the parameter $\beta_{0}$.

\section{References}

[1] P. A. Nelson, S. J. Elliott, Active Control of Sound, Academic, New York, 1992.

[2] R. Jones, On the theory of the directional patterns of continuous source distributions on a plane surface, Journal of Acoustical Society of America, 16(3), 147-171, 1945.

[3] W. F. Druyvesteyn, J. Garas, Personal sound, Journal of Audio Engineering Society, 45, 685-701, 1997. 
[4] J. Choi, Y. Kim, Generation of an acoustically bright zone with an illuminated region using multiple sources, Journal of Acoustical Society of America, 111(4), 1695-1700, 2002. [5] M. Shin, S. Lee, F. Fazi, P. A. Nelson, D. Kim, S. Wang, K. Park, J. Seo, Maximization of acoustic energy difference between two spaces, Journal of Acoustical Society of America, 128(1), 121-131, 2010.

[6] J. Chang, J. Park, Y. Kim, Scattering effect on the sound focused personal audio system, Journal of Acoustical Society of America, 125(5), 3060-3066, 2009.

[7] J. Chang, C. Lee, J. Park, Y. Kim, A realization of sound focused personal audio system using acoustic contrast control, Journal of Acoustical Society of America, 125(4), 2091-2097, 2009.

[8] S.J. Elliott, M. Jones, Active headrest for personal audio, Journal of Acoustical Society of America, 119, 2702-2709, 2006.

[9] S. J. Elliott, J. Cheer, H. Murfet, K.R. Holland, Minimally radiating sources for personal audio, Journal of Acoustical Society of America, 128(4), 1721-1728, 2010.

[10] S. J. Elliot, J. Cheer, J Choi, Y. Kim, Robustness and regularization of personal audio systems, IEEE Transaction on Audio, Speech and Language Processing, 20(7), 2123-2133, 2012.

[11] O. Kirkeby, P. A. Nelson, Reproduction of plane wave sound fields, Journal of Acoustical Society of America, 94(5), 2992-3000, 1993.

[12] J. L. Iturrioz, Inverse filter design for sound field control with loudspeaker line array systems, MS thesis, University of Southampton, 2011.

[13] F. Hirono, Miniaturized loudspeaker array for 3D audio reproduction, MS thesis, University of Southampton, 2012.

[14] J. Chang, F. Jacobsen, Sound field control with a circular double-layer array of loudspeakers, Journal of Acoustical Society of America, 131(6), 4518-4525, 2012. 
[15] F. M. Fazi, P. A. Nelson, Non-uniqueness of the solution of the sound field reproduction problem with boundary pressure control, Acta Acustica united with Acustica, 98(1), 1-14 2012

[16] O. Kirkeby, P. A. Nelson, H. Hamada, F. Orduna-Bustamante, Fast deconvolution of multichannel systems using regularization, IEEE Transaction on Speech and Audio Processing, 6(2), 189 194, 1998.

[17] HiVi Inc., Overview B1S Full-Frequency, accessed in 11 October 2012 at 19:00, URL http://www.swanspeaker.com/asp/product/htm/view.asp?id=119.

[18] E. Corteel, R. Pellegrini, C. Kuhn-Rahloff, Wave field synthesis with increased aliasing frequency, Proceedings on the 124th Convention of Audio Engineering Society, 7362, Amsterdam, 17 20 May, 2008.

[19] M. Zollner, E. Zwicker, Elektroakustik, Springer-Verlag, Berlin Heidelberg, 1933.

[20] ISVR, “ISVR Anechoic Chamber website,” accessed in 11 October 2012 at 19:00, URL http://www. southampton.ac.uk/engineering/outreach/activities/acoustic_test.page?

[21] P. C. Hansen, Rank-Deficient and Discrete Ill-posed Problems, SIAM, Philadelphia, 1998.

[22] P. Gauthier, C. Camier, O. Gauthier, Y. Pasco, A. Berry, Sound field reproduction of real flight recordings in cabin mock-up: Modified multichannel equalization, Proceedings on the 52nd International conference of Audio Engineering Society, Guilford, 2 4 September, 2013. [23] G. H. Golub, C. F. Van Loan, Matrix Computations, The Johns Hopkins University Press, Baltimore and London, 1996. 
Fig. 1. Definition of the parameters in the acoustical problem and its discretized simulation setup describing the bright ( $\odot$ ) and dark $(\boldsymbol{*})$ control points: $S_{b}$ and $S_{d}$ are the bright and dark zones corresponding to the sound radiating angles of $\theta_{b}$ and $\theta_{d}, p\left(\mathbf{x}_{m}^{b}\right)$ and $p\left(\mathbf{x}_{m}^{d}\right)$ are pressures at $\mathbf{x}_{m}^{b}$ and $\mathbf{x}_{m}^{d}$ in the bright and dark zones, $q\left(\mathbf{y}_{n}\right)$ is the source strength of a loudspeaker located at $\mathbf{y}_{n}$ and $Z\left(\mathbf{x}_{m}^{b} \mid \mathbf{y}_{n}\right)$ and $Z\left(\mathbf{x}_{m}^{d} \mid \mathbf{y}_{n}\right)$ are the acoustic transfer functions from the source at $\mathbf{y}_{n}$ to the pressures at $\mathbf{x}_{m}^{b}$ and $\mathbf{x}_{m}^{d}$.

Fig. 2. Photographs (side (a) and top (b) views) of the dual layer array with 16 loudspeakers mounted in an enclosure and experimental setup for the measurement of transfer functions and radiation patterns of the loudspeaker array in the ISVR anechoic chamber (c) and its schematics.

Fig. 3. Design of the desired signal $\hat{p}_{b}$ (-) in the bright zone: (a) frequency response amplitudes ( - : the pressure at the on-axis microphone in $3 \mathrm{~m}$ away when the 8 loudspeakers in the front layer are turned on), (b) phase and (c) impulse responses.

Fig. 4. Determination of the regularization parameter $\beta$ with constrained minimization approach; the solid line shows the input power of the loudspeaker array and the dashed line represents sound pressure level difference between bright and dark zones on a dB scale as a function of $\beta$. The excitation frequencies are (a) $125 \mathrm{~Hz}$, (b) $250 \mathrm{~Hz}$, (c) $500 \mathrm{~Hz}$, (d) 1000 
$\mathrm{Hz}$, (e) $2000 \mathrm{~Hz}$ and (f) $4000 \mathrm{~Hz}$. The arrows indicate the selected value of $\beta$ that yields a total input power of $5 \mathrm{~dB}$.

Fig. 5. Minimization of error in mean squared pressure: (a) frequency dependent regularization factors and (b) corresponding total input powers. ----- represents the $\beta$ calculated with Eq. (5), - represents the selected $\beta$ based on the procedure in Fig. 4.

Fig. 6. Minimization of error in mean squared pressure: simulated averaged radiation patterns with $\beta$ s selected (-) and calculated (-----) by Eq. (5): (a) $88 \sim 177 \mathrm{~Hz}$, (b) $177 \sim 355 \mathrm{~Hz}$, (c) $355 \sim 710 \mathrm{~Hz}$, (d) $710 \sim 1420 \mathrm{~Hz}$, (e) $1420 \sim 2840 \mathrm{~Hz}$ and (f) $2840 \sim 8000 \mathrm{~Hz}$.

Fig. 7. Minimization of error in mean squared pressure: frequency response functions $(a, b, c)$ along with phase angles (d, e, f) and time domain impulse responses $(g, h, i)$ at the control points, $1(\mathrm{a}, \mathrm{d}, \mathrm{g}), 3(\mathrm{~b}, \mathrm{e}, \mathrm{h})$ and $5(\mathrm{c}, \mathrm{f}, \mathrm{i})$ in the bright zone. The desired signal $(-)$, control results with $\beta$ s selected (-) and calculated (-----) by the Eq. (5) have been overlaid.

Fig. 8. The $\alpha$ decision procedure; — shows the averaged sound pressure in the bright zone and ----- represents the sound pressure level difference between bright and dark zones on a $\mathrm{dB}$ scale with respect to the change of $\alpha$ value. The excitation frequencies are (a) $125 \mathrm{~Hz}$, (b) $250 \mathrm{~Hz}$, (c) $500 \mathrm{~Hz}$, (d) $1000 \mathrm{~Hz}$, (e) $2000 \mathrm{~Hz}$ and (f) $4000 \mathrm{~Hz}$. The arrows indicate the selected $\alpha_{q}$ (preserving the averaged sound pressure in the bright zone as $20 \mathrm{~dB}$ ) and $\alpha_{c}$ (preserving the maximum sound pressure difference between bright and dark zones). 
Fig. 9. Maximization of energy difference: (a) frequency dependent tuning factors, $\alpha$ and (b) corresponding total input powers. — represents $\alpha_{q}$ and ----- represents $\alpha_{c}$ selected based on the procedure in Fig. 8.

Fig. 10. Maximization of energy difference: simulated averaged radiation patterns with $\alpha_{q}$ $(-)$ and $\alpha_{c}(-----)$ within 6 frequency bands.

Fig. 11. Maximization of energy difference: frequency response functions (a, b, c) along with phase angles (d, e, f) and time domain impulse responses $(g, h, i)$ at the control points, 1 (a, d, g), 3 (b, e, h) and 5 (c, f, i) in the bright zone. The desired signal (-), control results with $\alpha_{q}$ (-) and $\alpha_{c}(----)$ have been overlaid.

Fig. 12. Minimization of weighted pressure error and energy: total input powers with $\kappa=0.9 \quad(-)$ and $\kappa=0.5 \quad(----)$.

Fig. 13. Minimization of weighted pressure error and energy: simulated averaged radiation patterns with $\kappa=0.9(-)$ and $\kappa=0.5 \quad(----)$ within 6 frequency bands.

Fig. 14. Minimization of weighted pressure error and energy: frequency response functions (a, b, c) along with phase angles (d, e, f) and time domain impulse responses (g, h, i) at the control points, 1 (a, d, g), 3 (b, e, h) and 5 (c, f, i) in the bright zone. The desired signal (-), control results with $\kappa=0.9$ (-) and $\kappa=0.5(----)$ have been overlaid. 
Fig. 15. Definition of the parameters in a modified acoustical problem examined: $S_{b}, S_{g}$ and $S_{d}$ are the bright, gray and dark zones corresponding to the sound radiating angles of $\theta_{b}, \theta_{g}$ and $\theta_{d}, p\left(\mathbf{x}_{m}^{b}\right), p\left(\mathbf{x}_{m}^{g}\right)$ and $p\left(\mathbf{x}_{m}^{d}\right)$ are pressures at $\mathbf{x}_{m}^{b}, \mathbf{x}_{m}^{g}$ and $\mathbf{x}_{m}^{d}$ in the bright, gray and dark zones, $q\left(\mathbf{y}_{n}\right)$ is the source strength of a loudspeaker located at $\mathbf{y}_{n}$ and $Z\left(\mathbf{x}_{m}^{b} \mid \mathbf{y}_{n}\right)$, $Z\left(\mathbf{x}_{m}^{g} \mid \mathbf{y}_{n}\right)$ and $Z\left(\mathbf{x}_{m}^{d} \mid \mathbf{y}_{n}\right)$ are the acoustic transfer functions from the source at $\mathbf{y}_{n}$ to the pressures at $\mathbf{x}_{m}^{b}, \mathbf{x}_{m}^{g}$ and $\mathbf{x}_{m}^{d}$.

Fig. 16. Two control point arrangements: array of loudspeakers surrounded by control points within the bright $(\circ)$, gray $(\bullet)$ and dark $(\boldsymbol{x})$ zones.

Fig. 17. Weighted minimization with a low priority zone with two control point arrangements in FIG. 19: total input powers with $\gamma=1 / 16$ (-) and $\gamma=1 / 2$ (-----).

Fig. 18. Weighted minimization with a low priority zone with control point arrangement 1 : simulated averaged radiation patterns with $\gamma=1 / 16$ (-) and $\gamma=1 / 2$ (-----) within 6 frequency bands.

Fig. 19. Weighted minimization with a low priority zone with control point arrangement 1 : frequency response functions $(a, b, c)$ along with phase angles $(d, e, f)$ and time domain impulse responses $(\mathrm{g}, \mathrm{h}, \mathrm{i})$ at the control points, $1(\mathrm{a}, \mathrm{d}, \mathrm{g}), 3(\mathrm{~b}, \mathrm{e}, \mathrm{h})$ and $5(\mathrm{c}, \mathrm{f}, \mathrm{i})$ in the bright zone. The desired signal (-), control results with $\gamma=1 / 16 \quad(-)$ and $\gamma=1 / 2$ (---- 
-) have been overlaid.

Fig. 20. Weighted minimization with a low priority zone with control point arrangement 2: simulated averaged radiation patterns with $\gamma=1 / 16$ (-) and $\gamma=1 / 2$ (-----) within 6 frequency bands.

Fig. 21. Weighted minimization with a low priority zone with control point arrangement 2: frequency response functions $(a, b, c)$ along with phase angles $(d, e, f)$ and time domain impulse responses $(\mathrm{g}, \mathrm{h}, \mathrm{i})$ at the control points, $1(\mathrm{a}, \mathrm{d}, \mathrm{g}), 3(\mathrm{~b}, \mathrm{e}, \mathrm{h})$ and $5(\mathrm{c}, \mathrm{f}, \mathrm{i})$ in the bright zone. The desired signal (-), control results with $\gamma=1 / 16 \quad(-)$ and $\gamma=1 / 2$ (----) have been overlaid.

Fig. 22. Experimental verifications (-----) of the simulated radiation patterns (-) controlled by weighted minimization with a low priority zone when $\gamma=1 / 16$ with control point arrangement 2 averaged within 6 frequency bands.

Fig. 23. Experimental verifications (-----) of the simulated (-) frequency response functions $(\mathrm{a}, \mathrm{b}, \mathrm{c})$ along with phase angles $(\mathrm{d}, \mathrm{e}, \mathrm{f})$ and time domain impulse responses $(\mathrm{g}, \mathrm{h}, \mathrm{i})$ at the control points, 1 (a, d, g), 3 (b, e, h) and 5 (c, f, i) in the bright zone, controlled by weighted minimization with a low priority zone when $\gamma=1 / 16$ with control point arrangement 2

Fig. 24. (color online) Averaged contrast comparisons when the arrangements of control points have been defined as (a) Fig. 1(b) and (b) Fig. 16 (a) in six frequency bands ( $1^{\text {st }}$ band: 
$88 \sim 177 \mathrm{~Hz}, 2^{\text {nd }}$ band: $177 \sim 355 \mathrm{~Hz}, 3^{\text {rd }}$ band: $355 \sim 710 \mathrm{~Hz}, 4^{\text {th }}$ band: $710 \sim 1420 \mathrm{~Hz}, 5^{\text {th }}$ band: $1420 \sim 2840 \mathrm{~Hz}, 6^{\text {th }}$ band: $2840 \sim 8000 \mathrm{~Hz}$ )

Fig. 25. (color online) Averaged mismatches of frequency response functions (FRF) in six frequency bands.

Fig. 26. (color online) Averaged efficiency of the loudspeaker array in six frequency bands. 


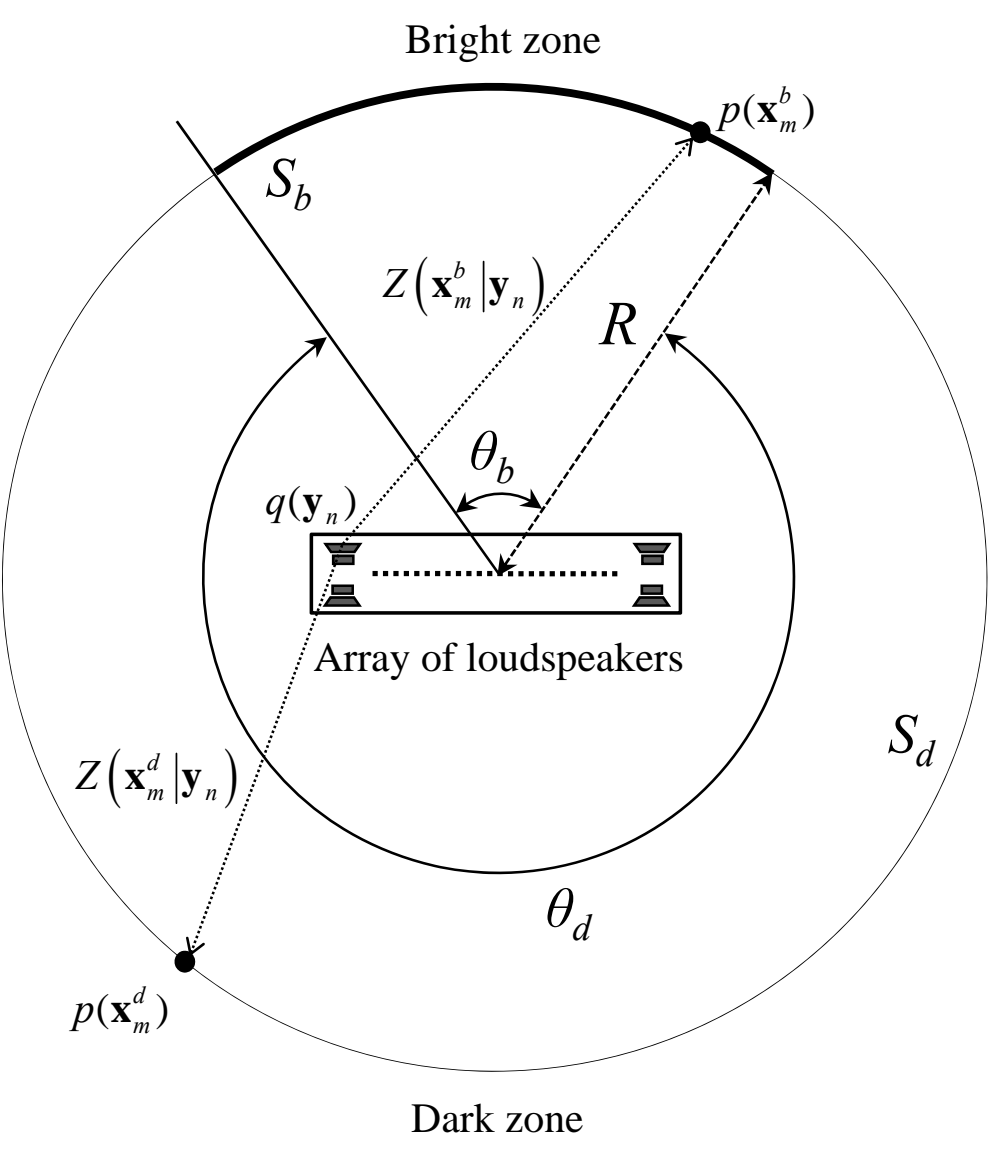

(a)

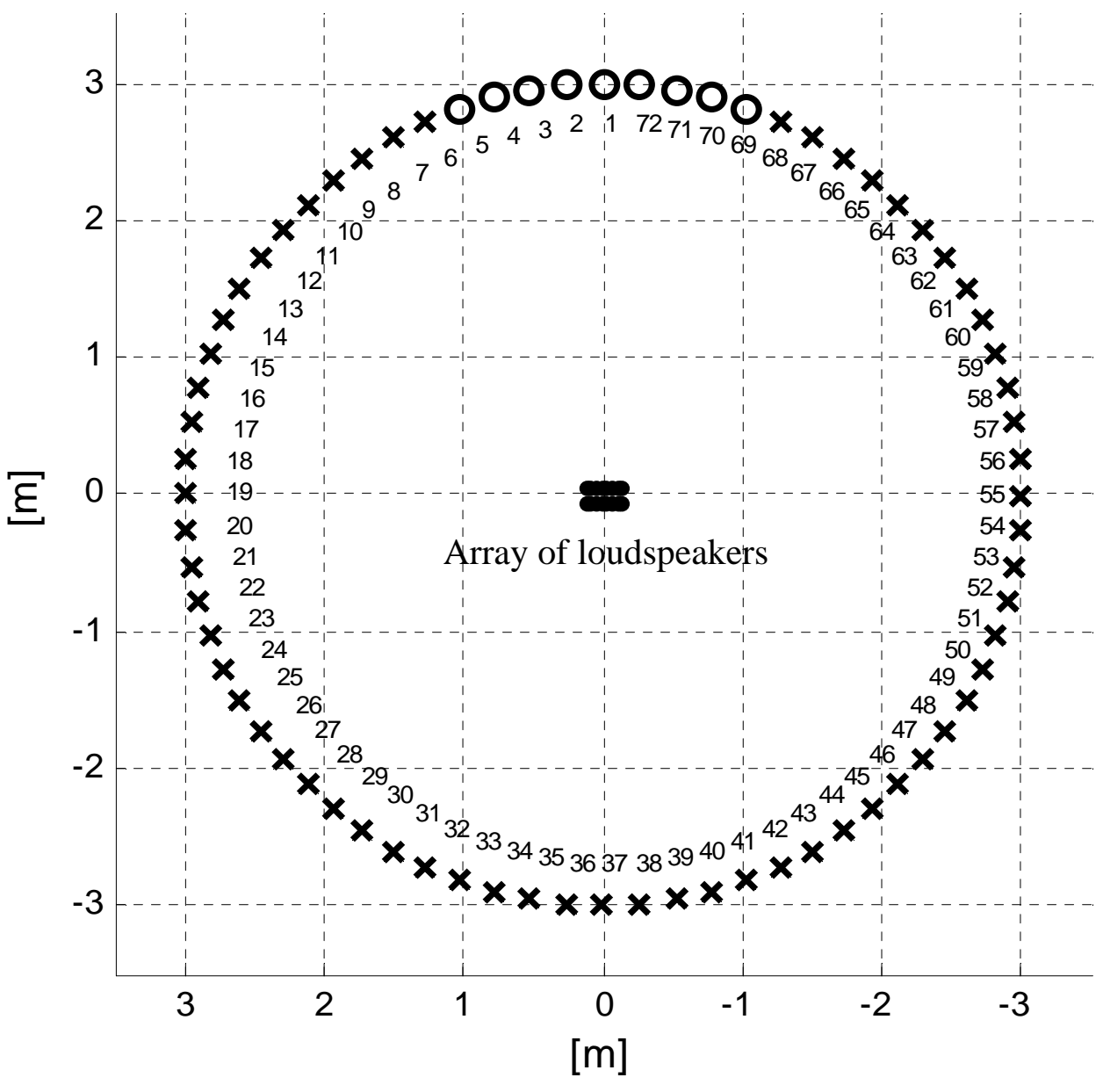

(b) 
Figure 2

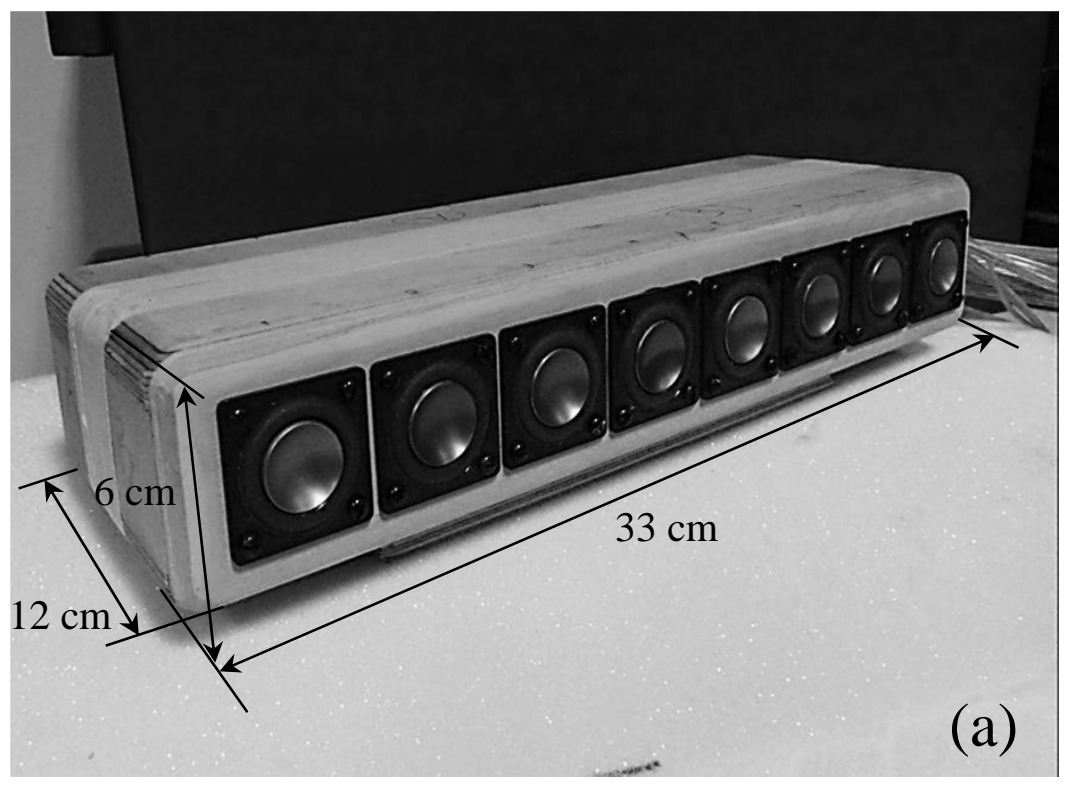
X)
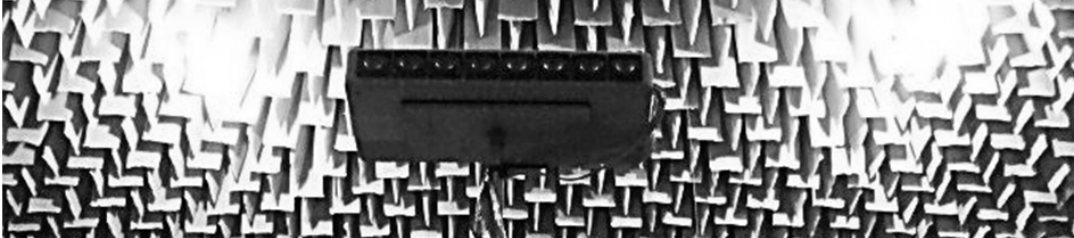

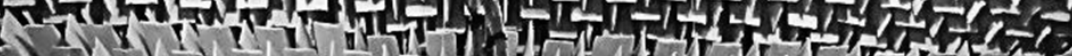

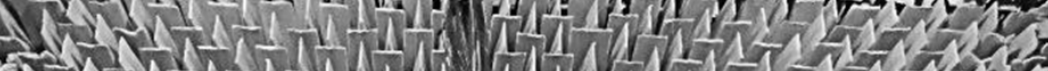

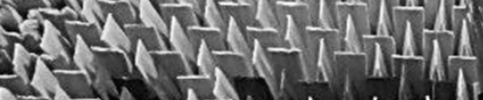

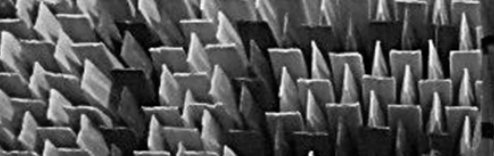

h 1

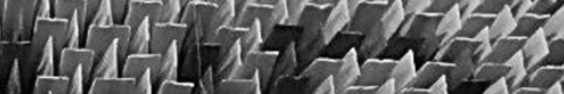

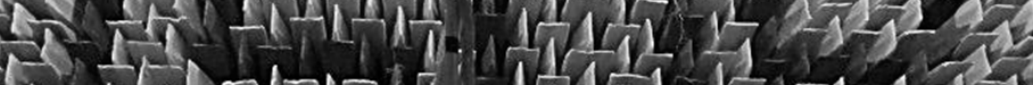

(a)

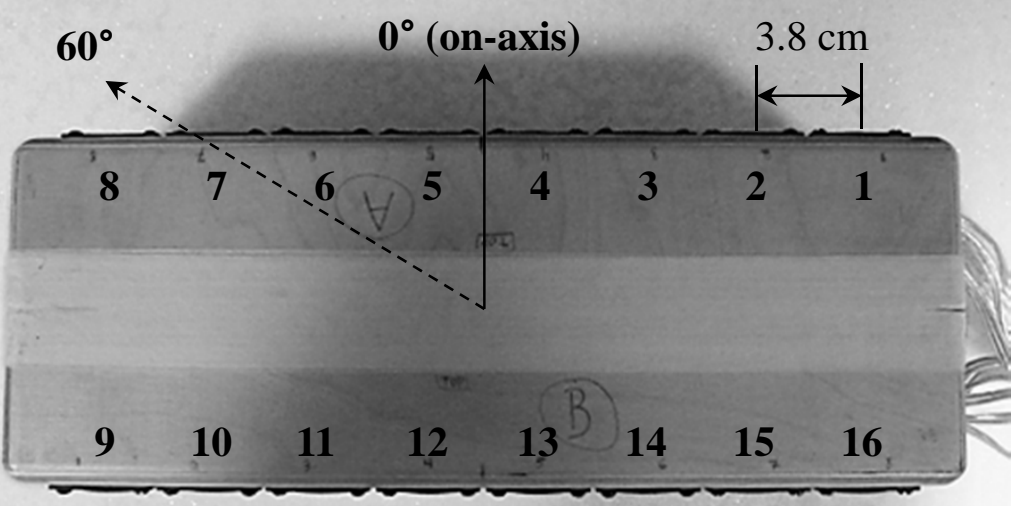

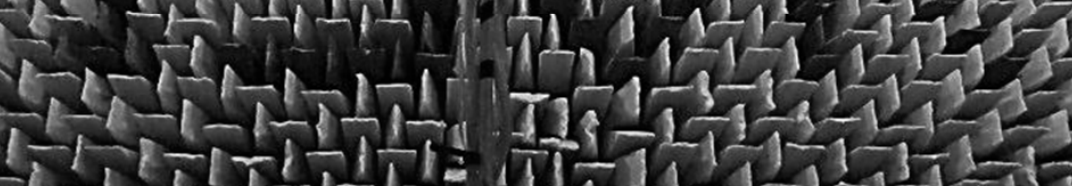

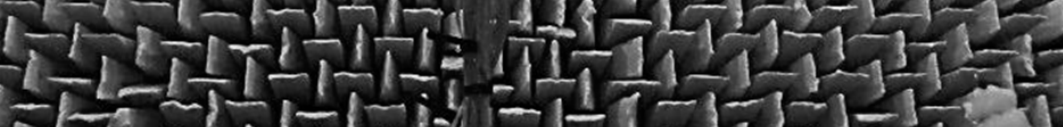
$-1-1-1-1 \div 1-1 ;$

(b)

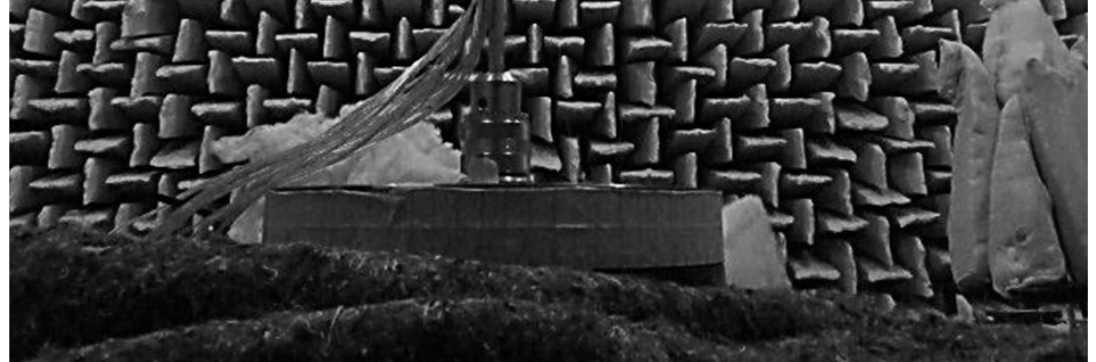

(c) 


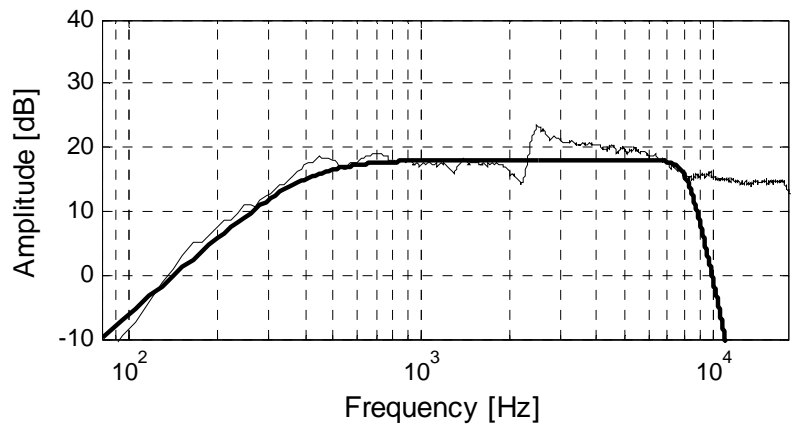

(a)

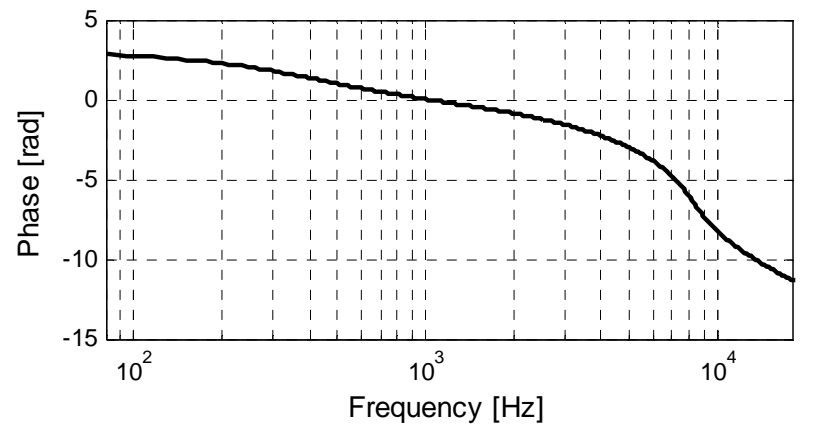

(b)

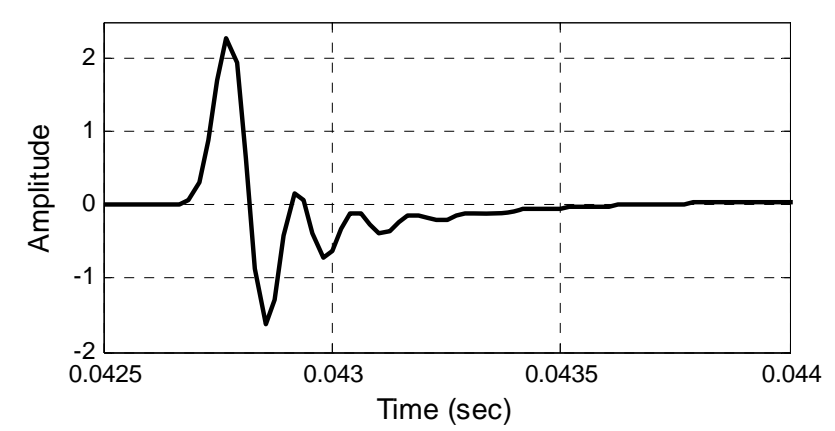

(c) 
Figure 4
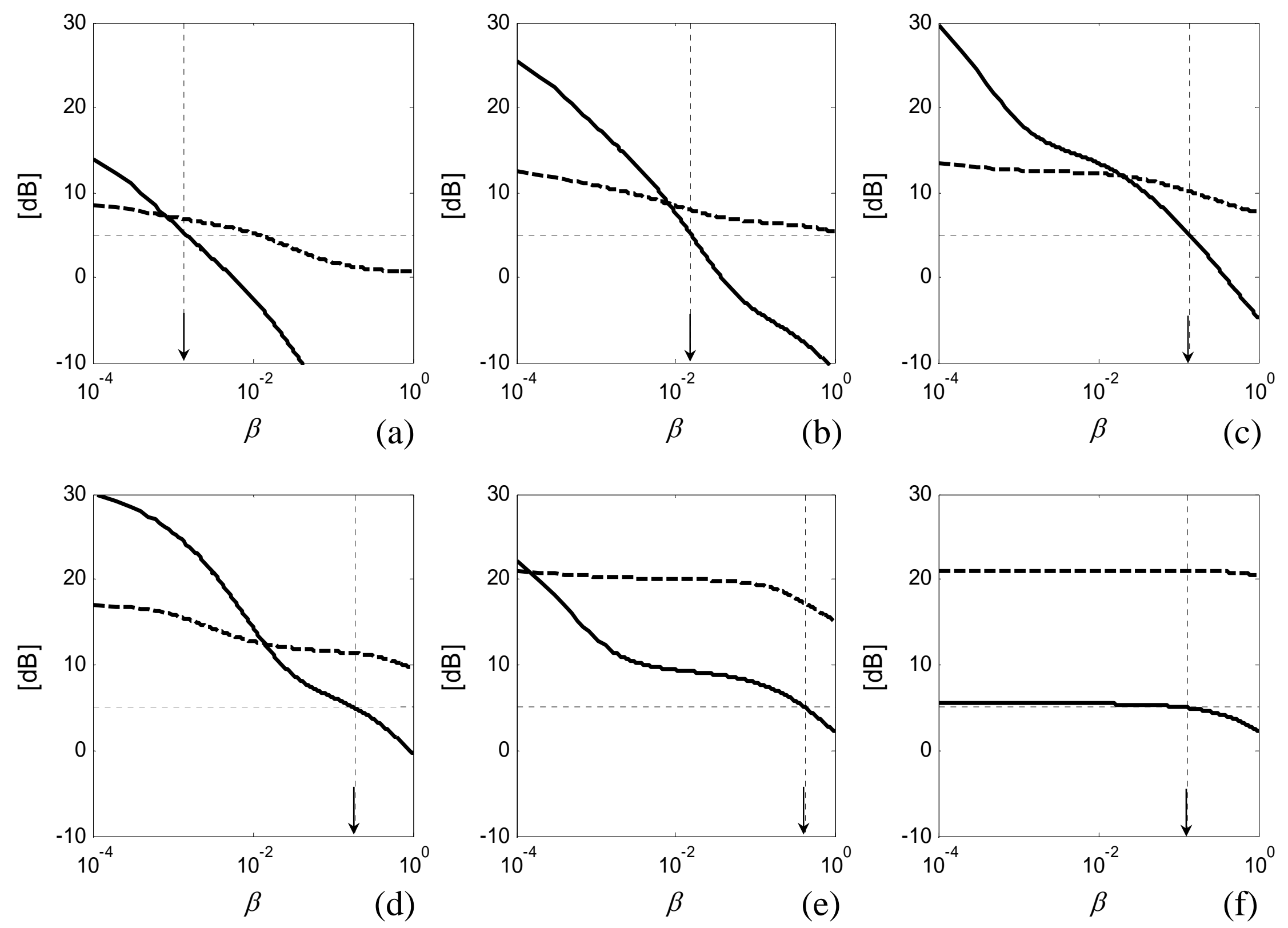


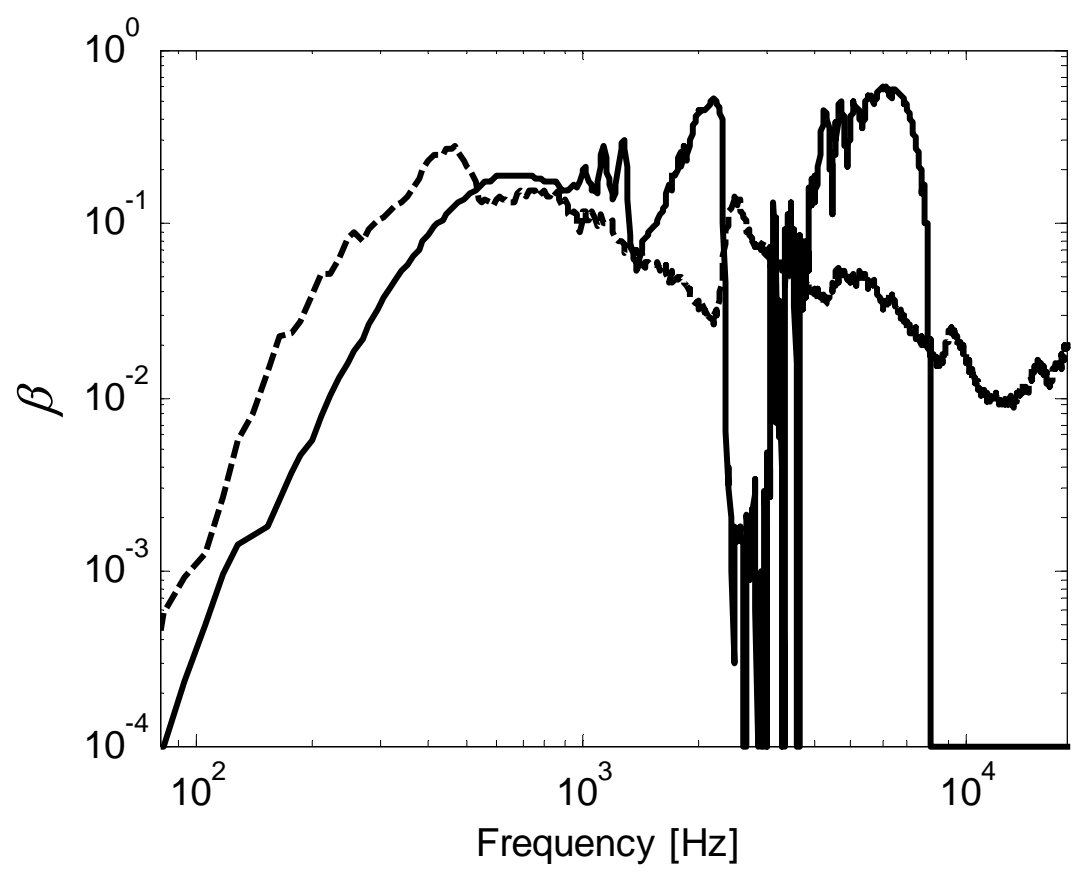

(a)

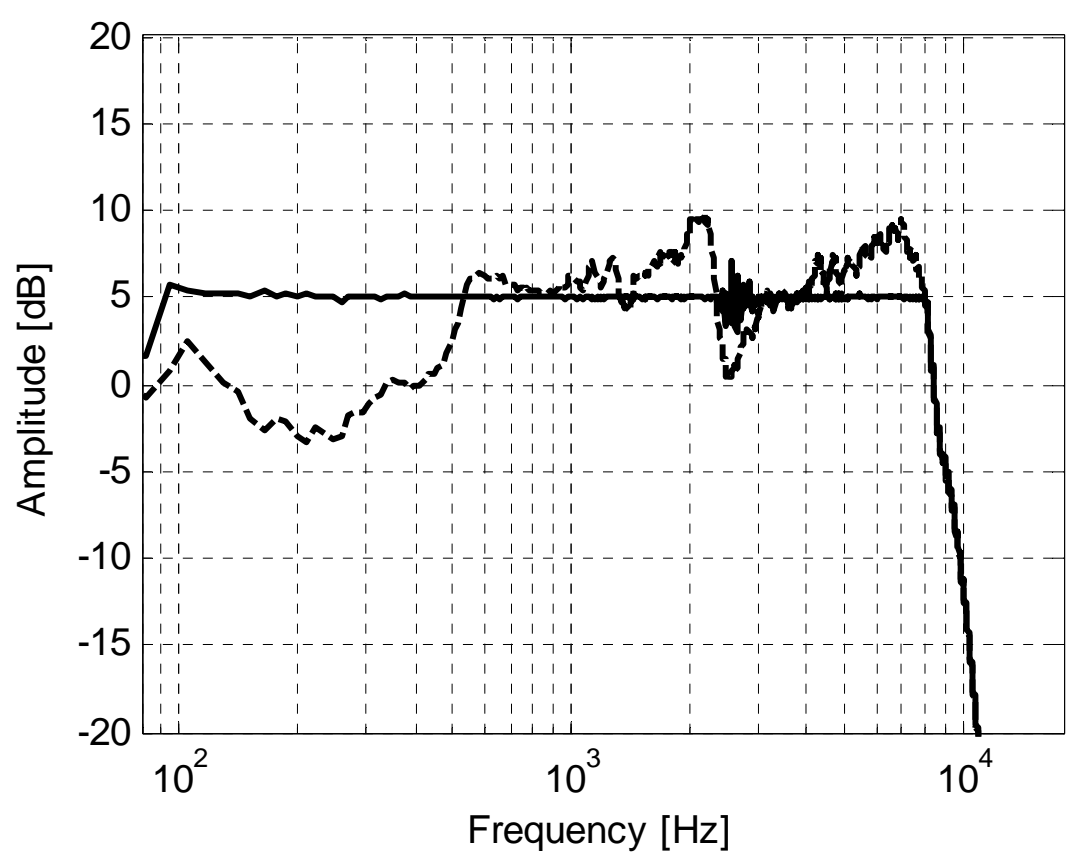

(b) 
Figure 6

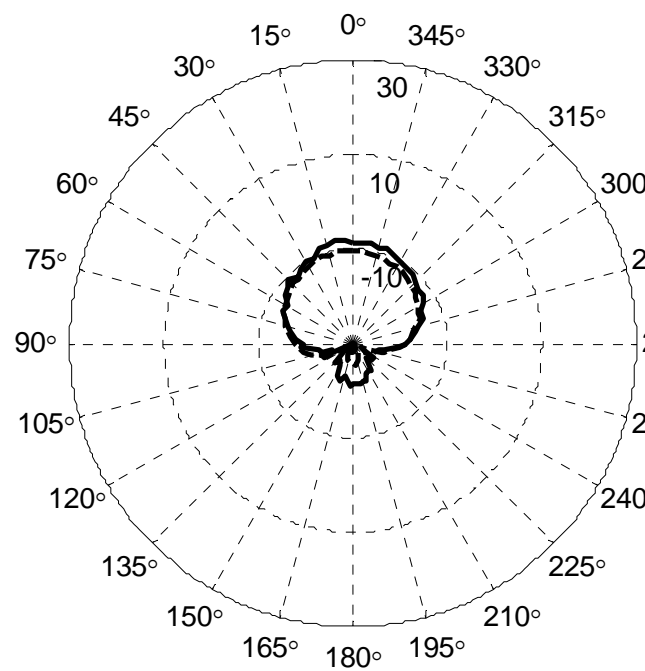

(a)

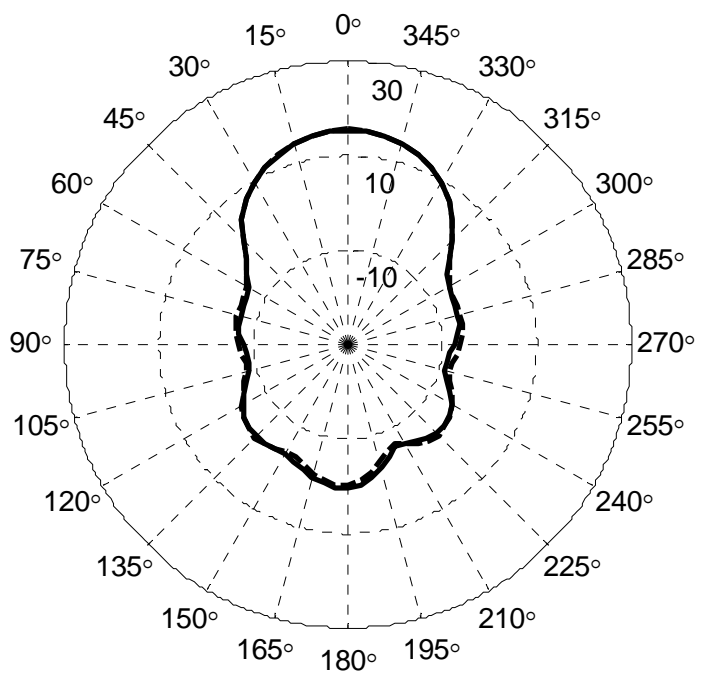

(d)

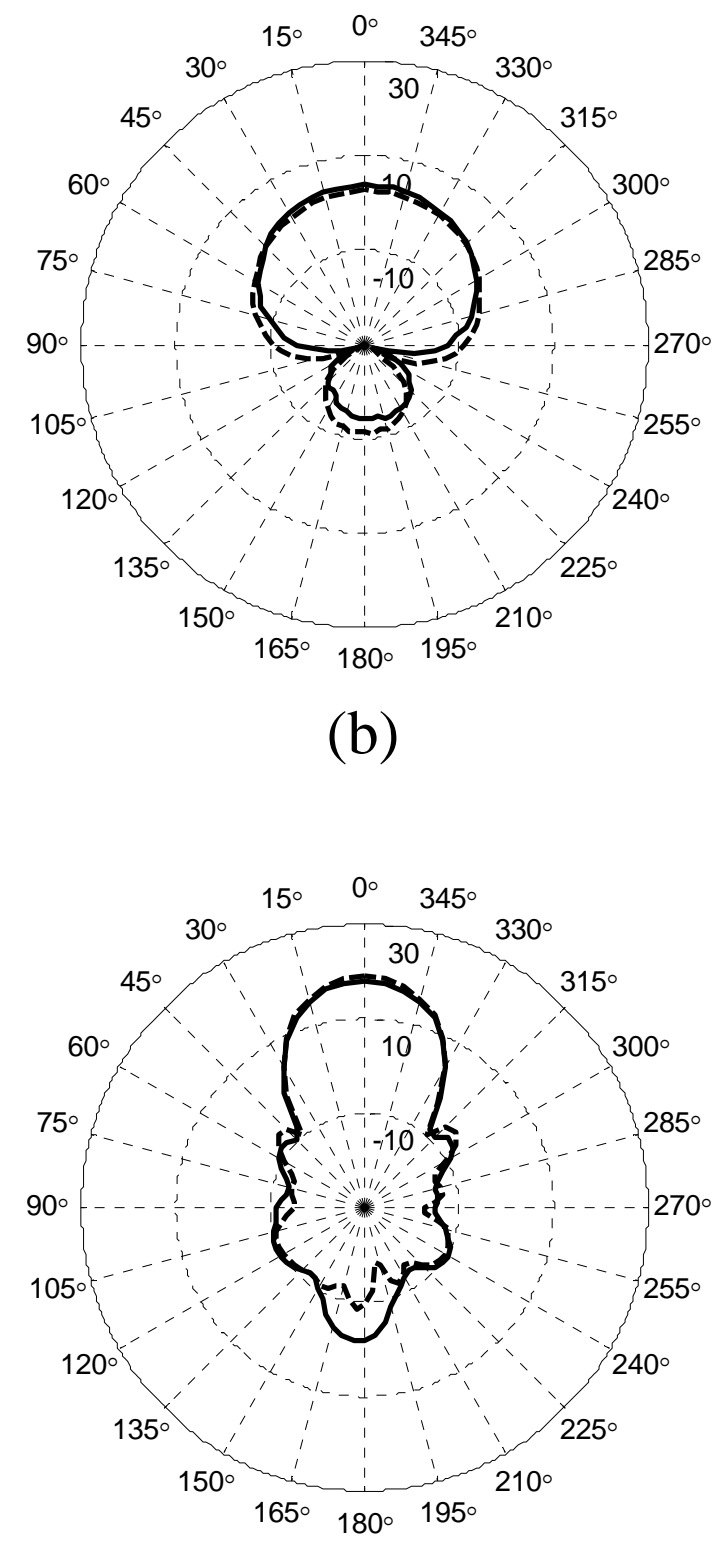

(e)

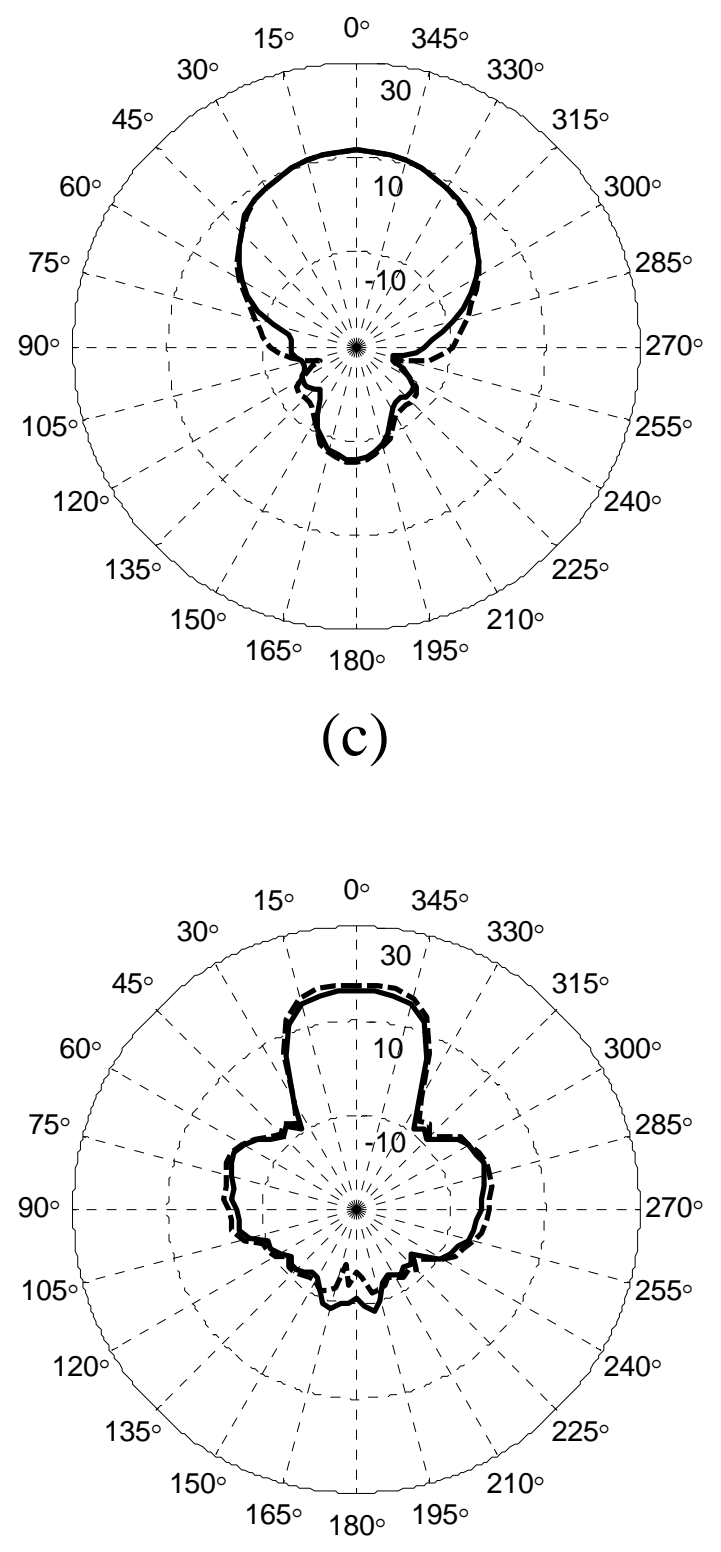

(f) 
1
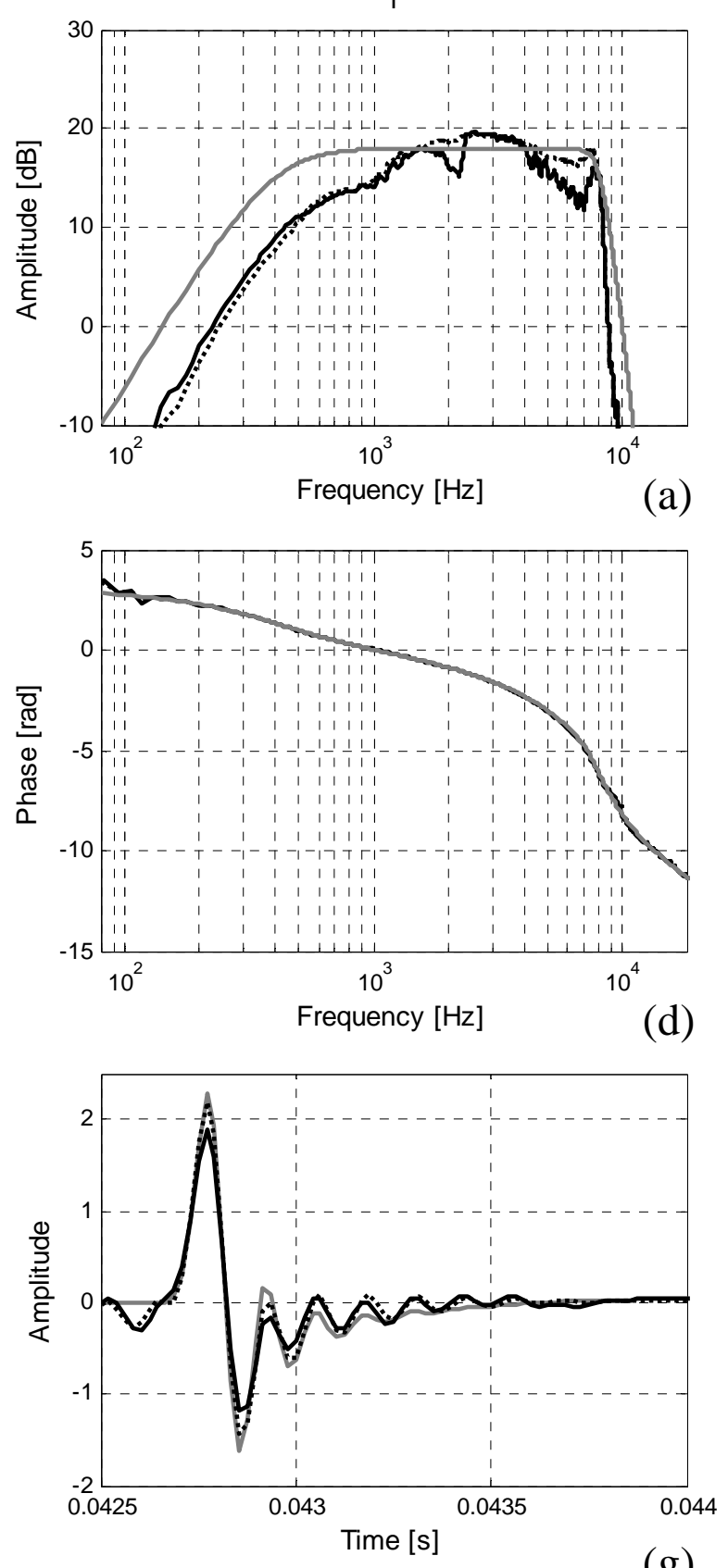

3
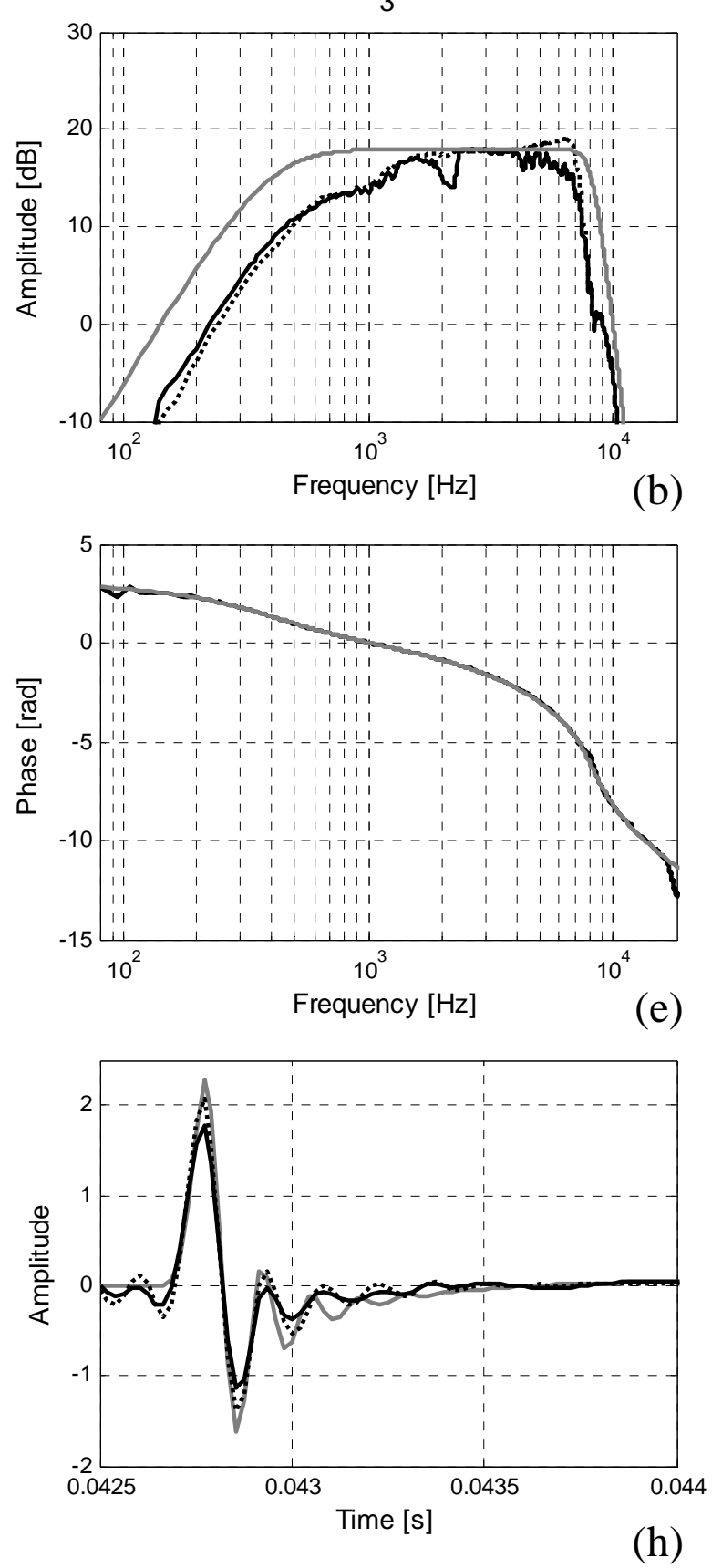

5
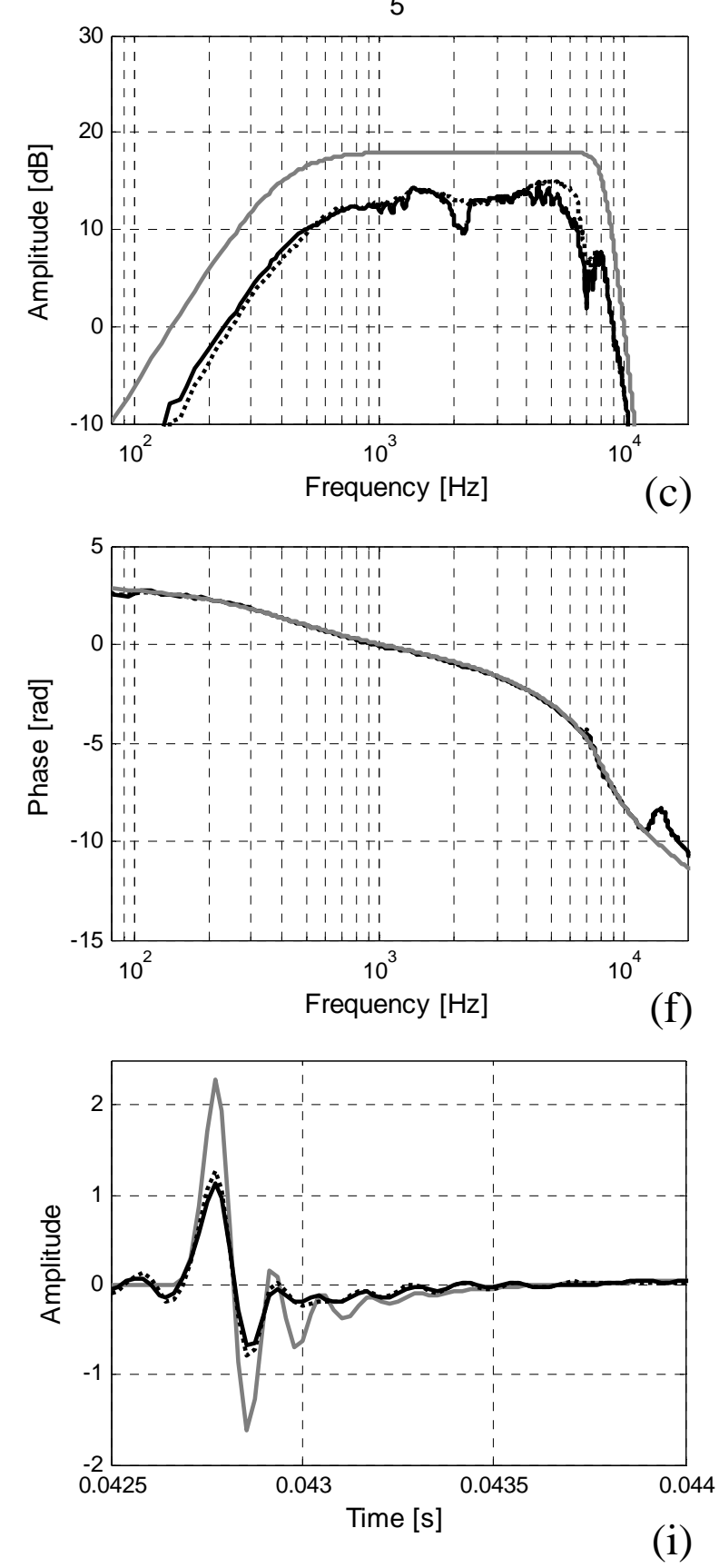

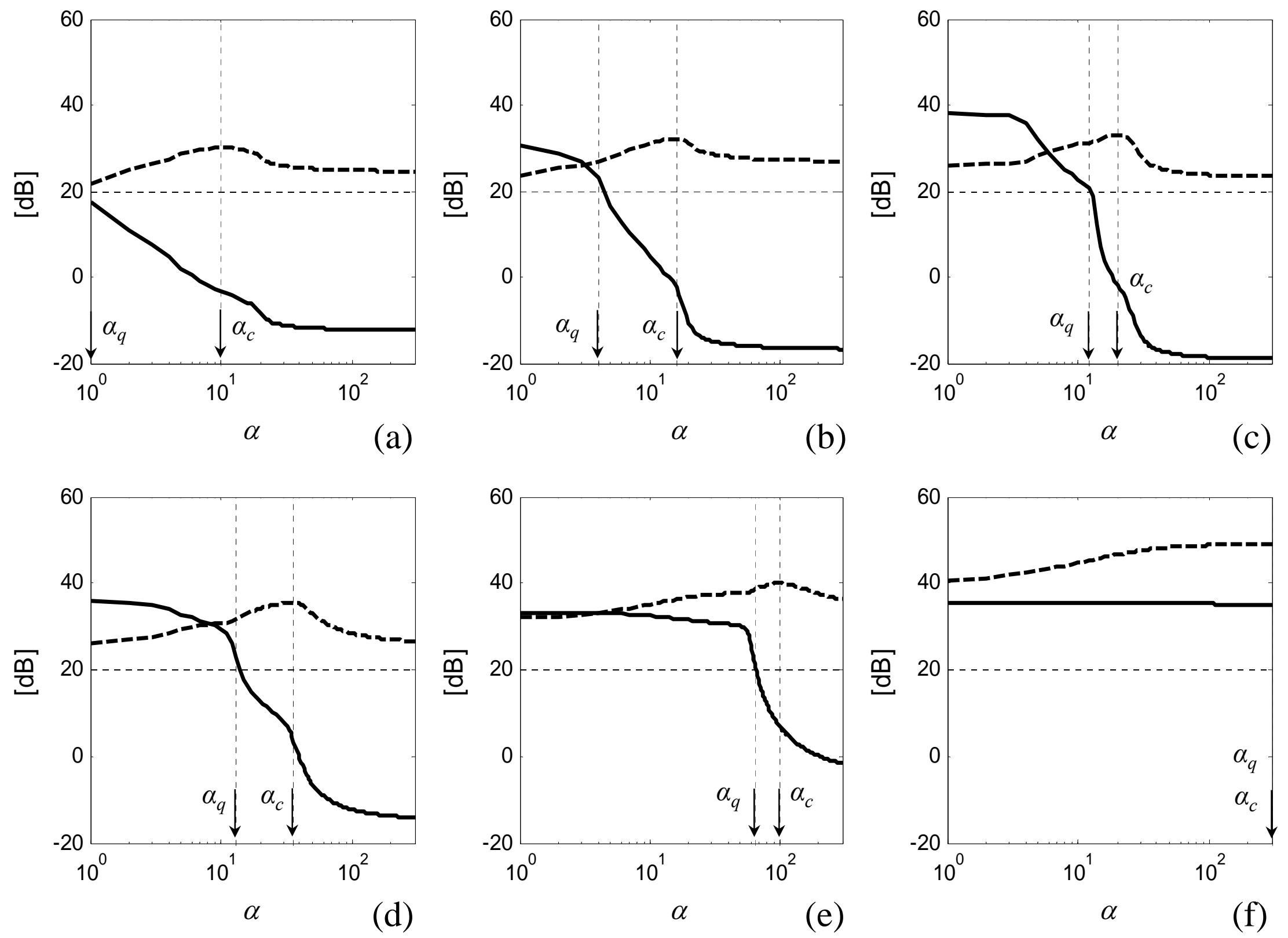


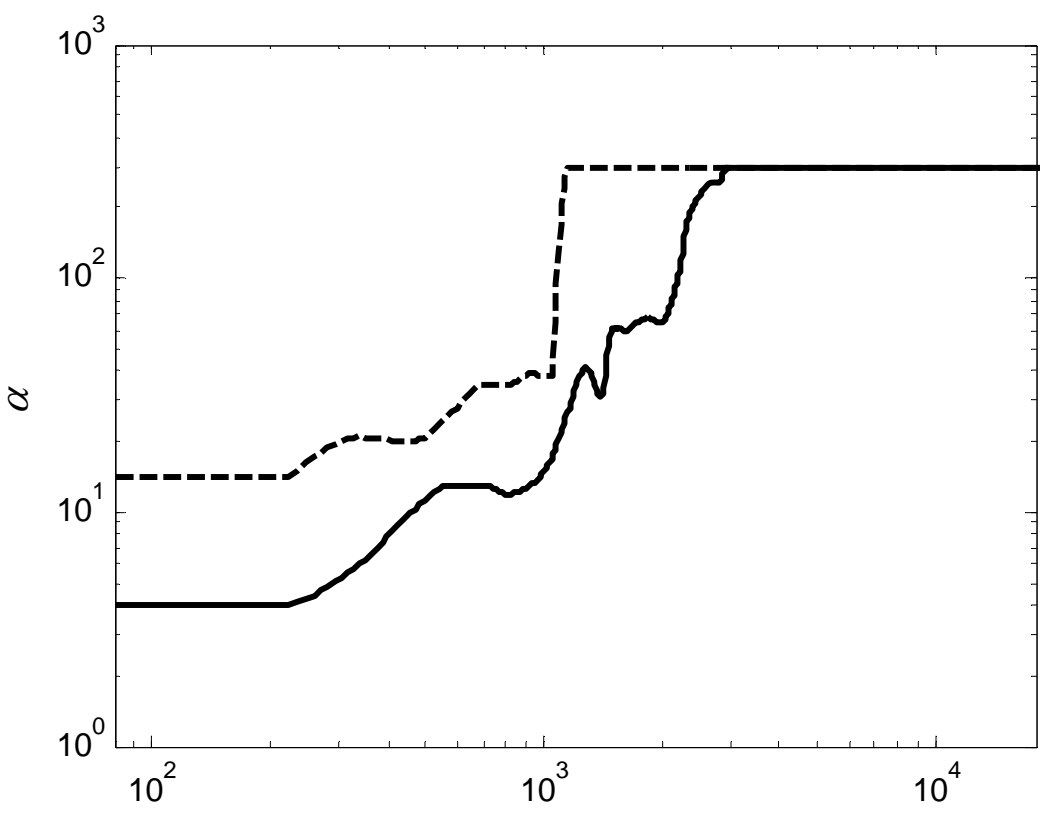

Frequency [Hz]

(a)

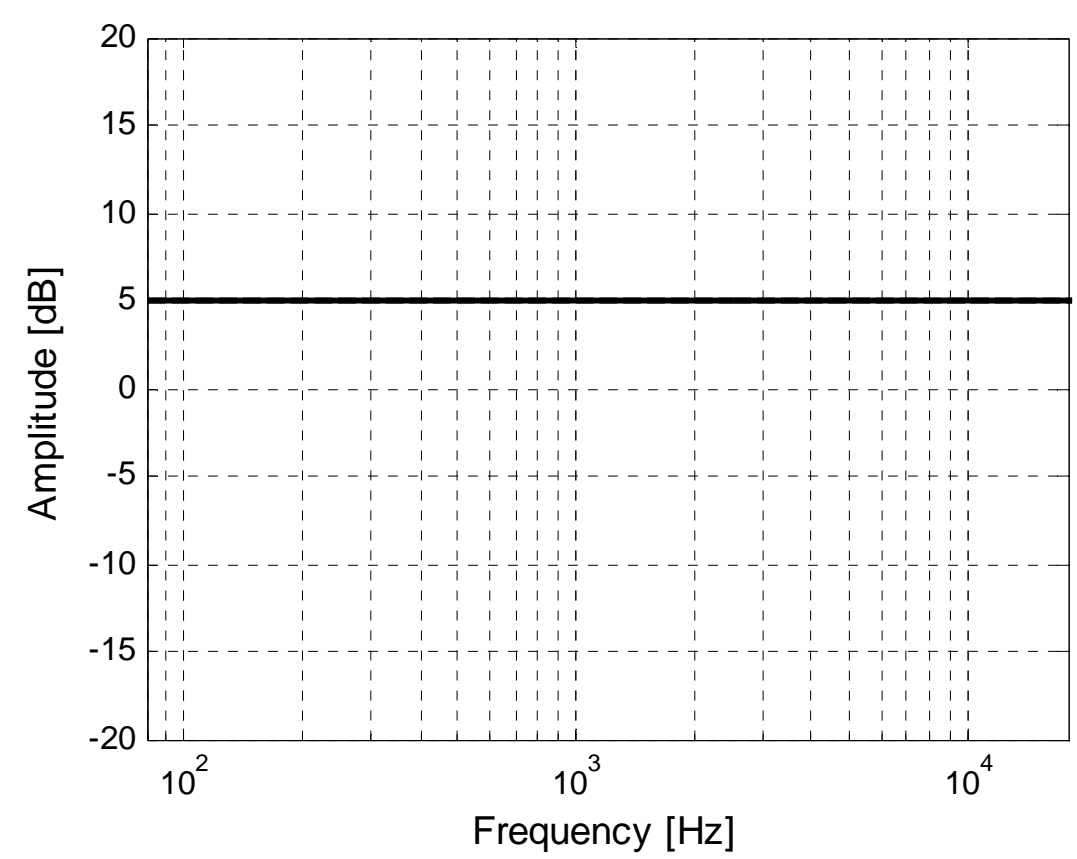

(b) 


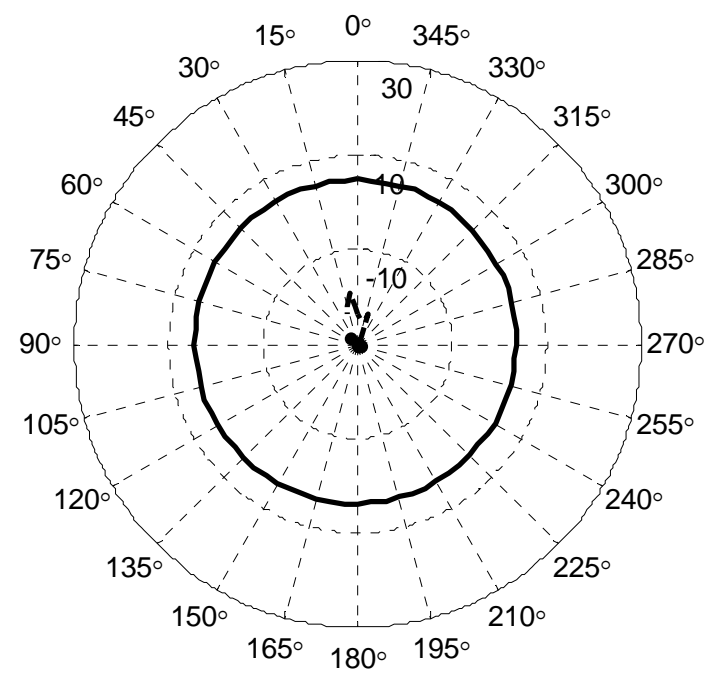

(a)

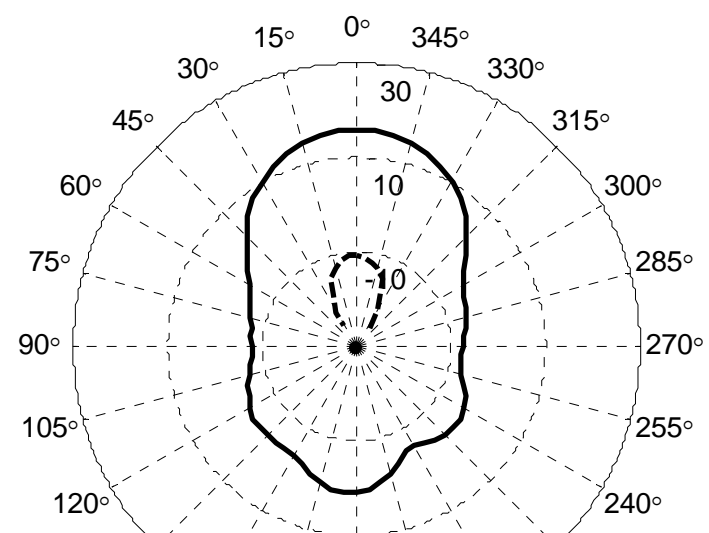

$135^{\circ}$

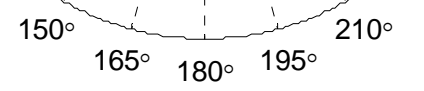

(d)

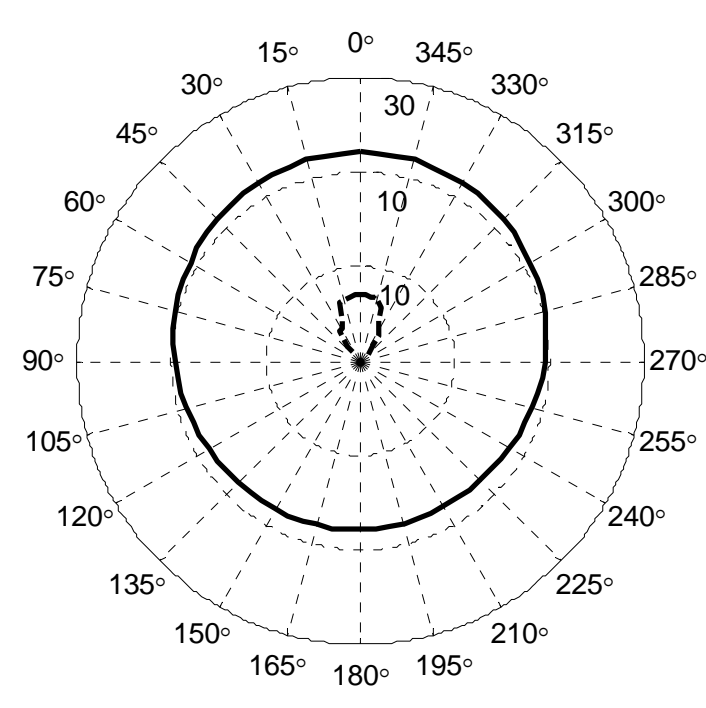

(b)
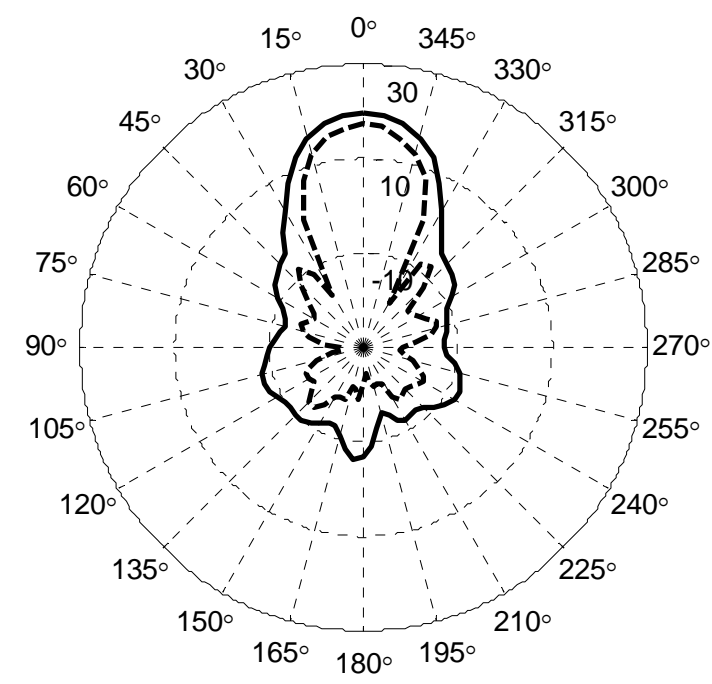

(e)

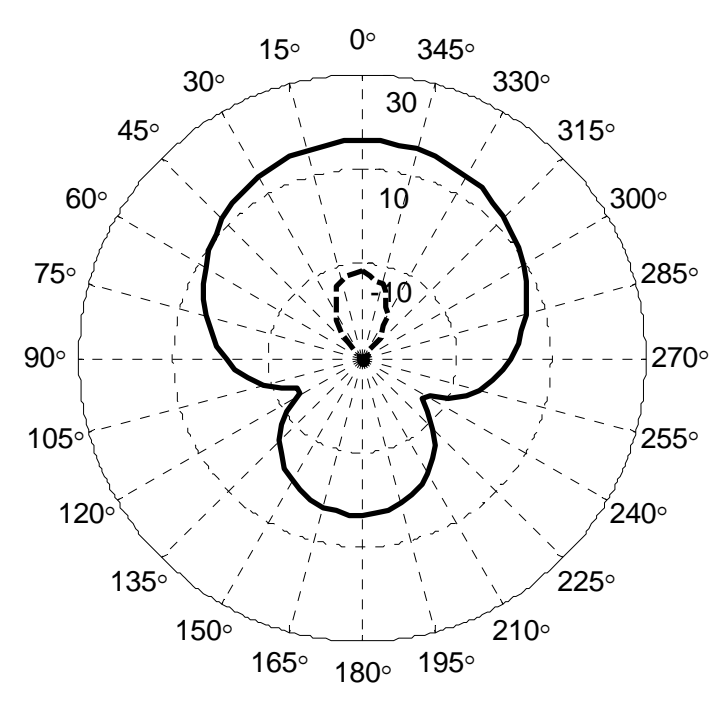

(c)

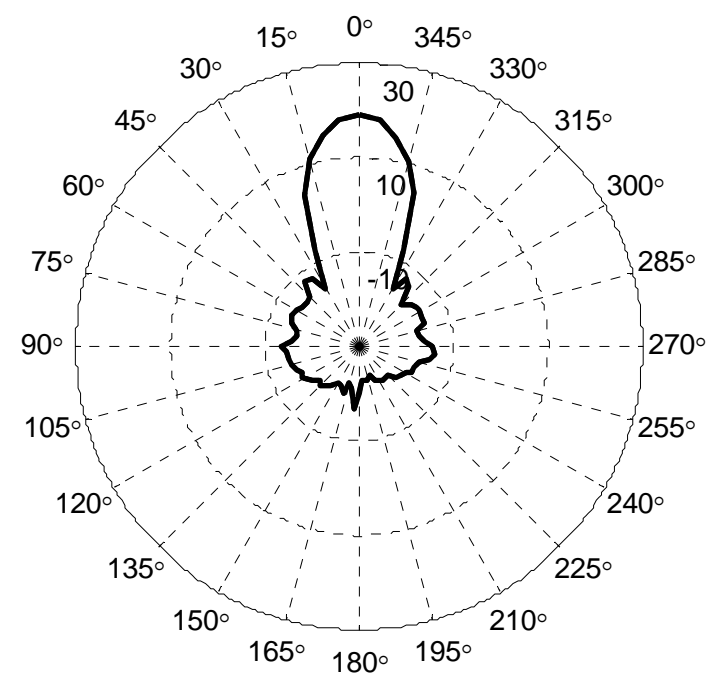

(f) 
1
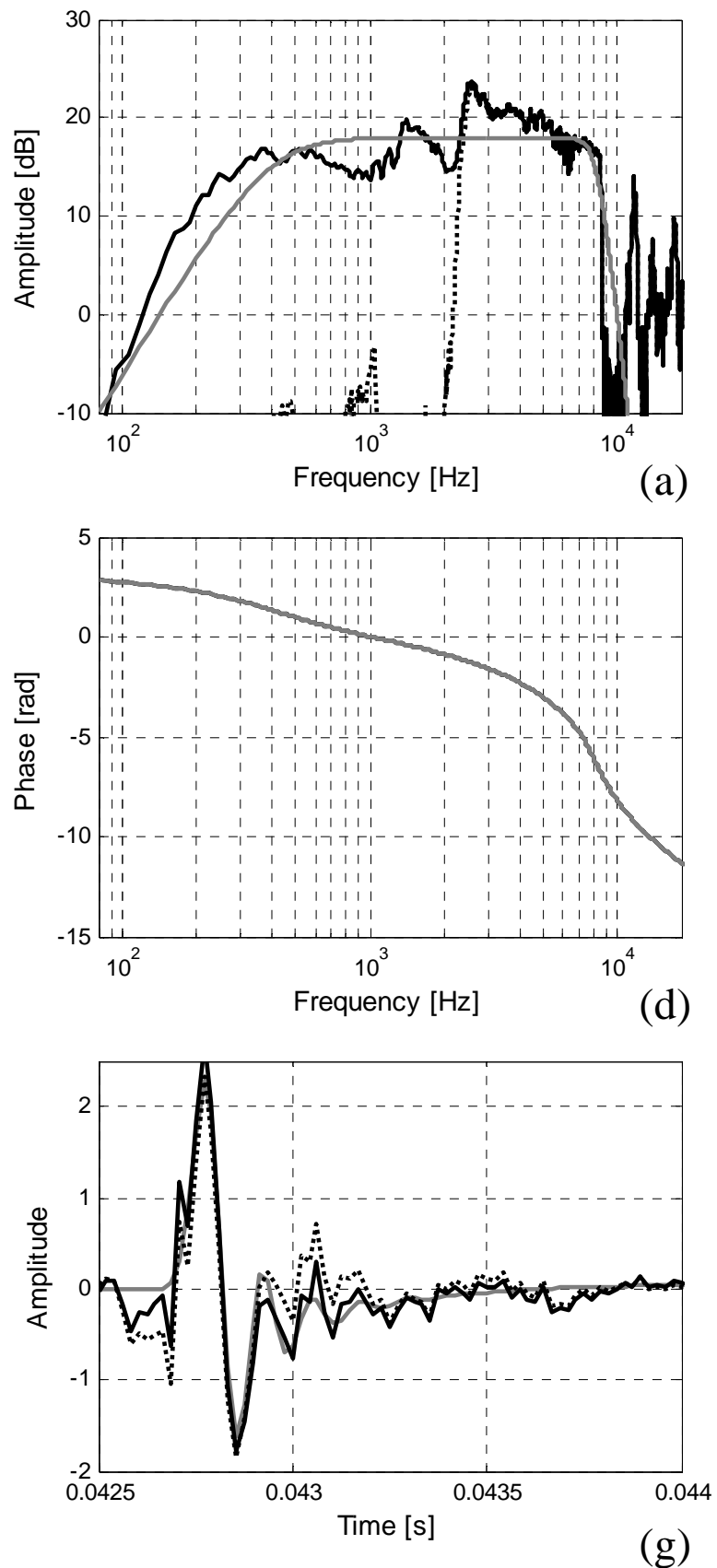

3
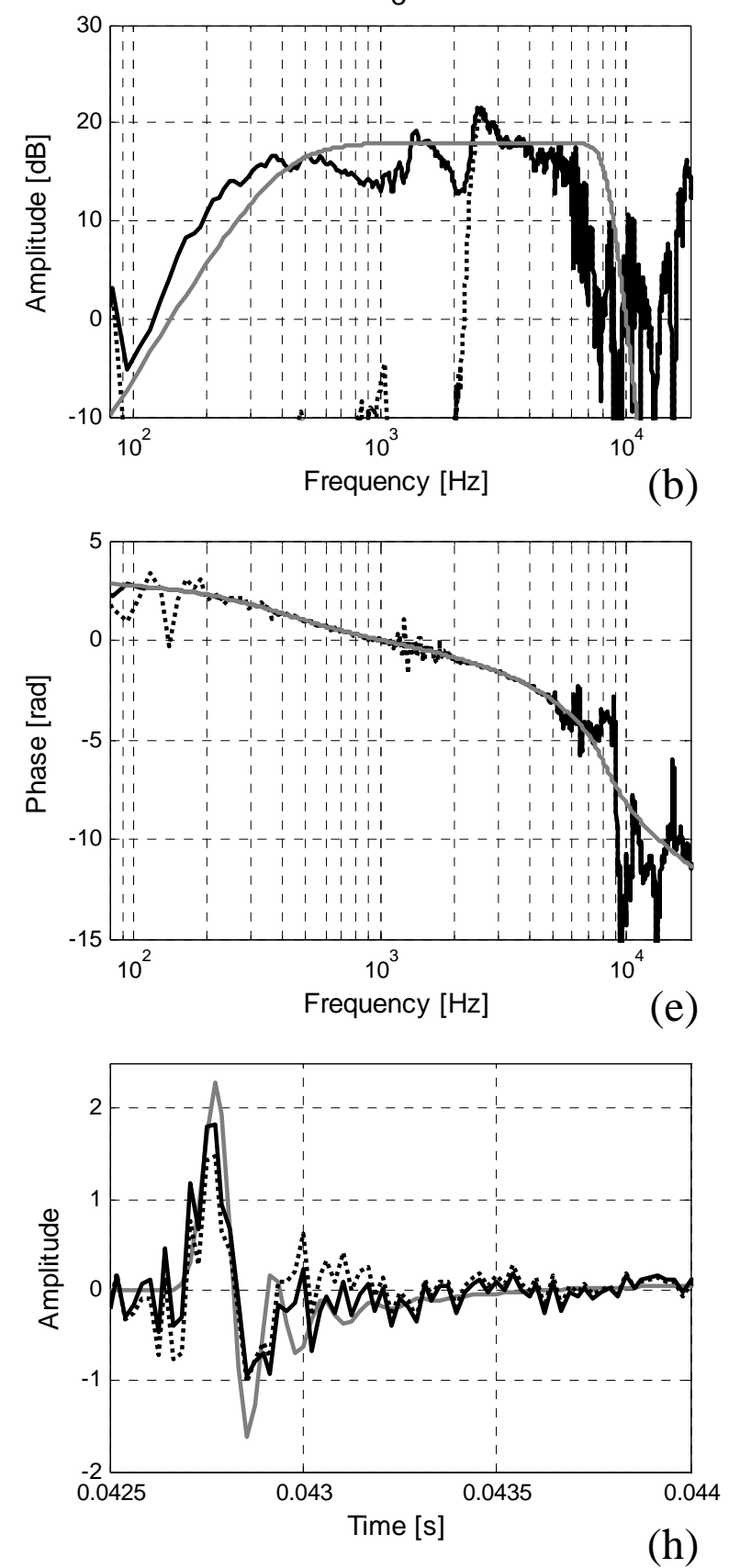

5
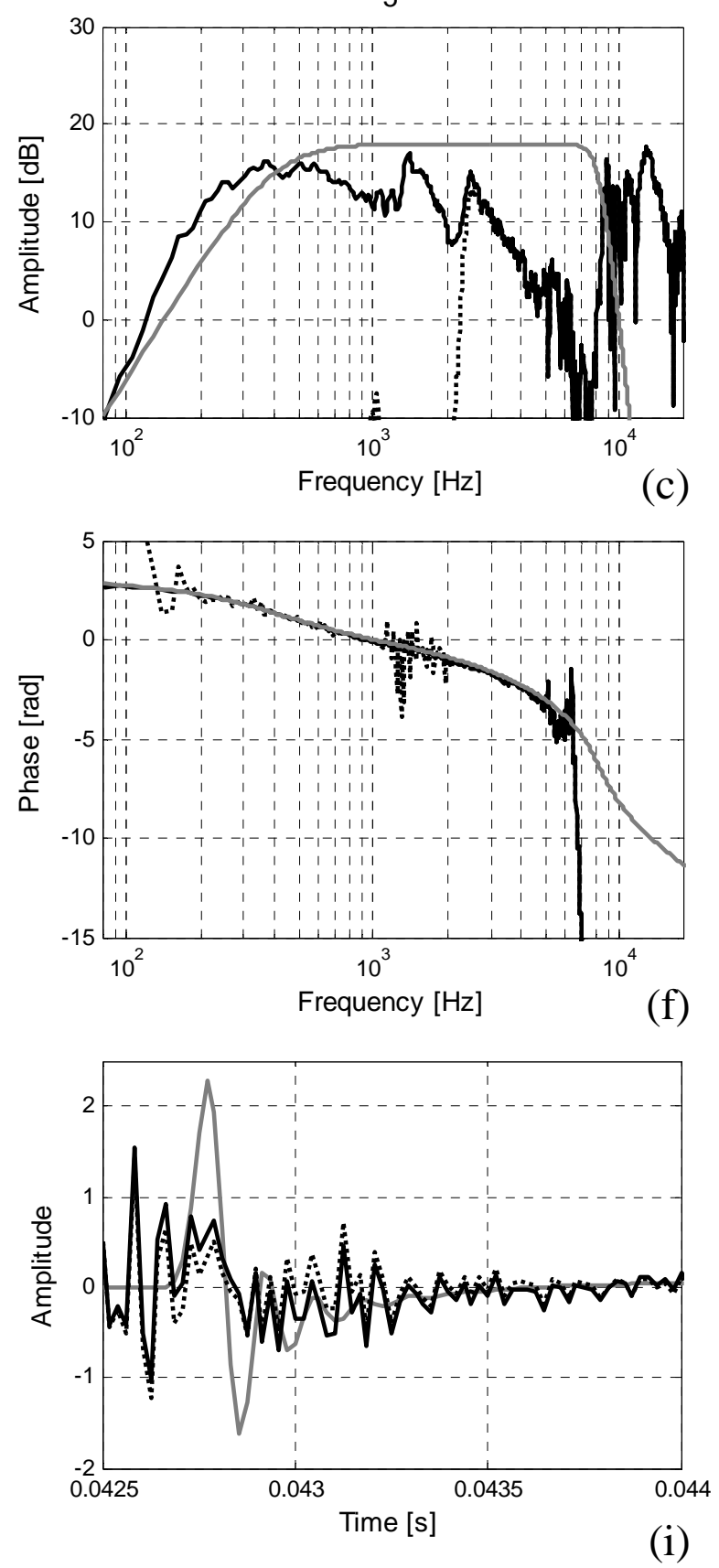
Figure 12

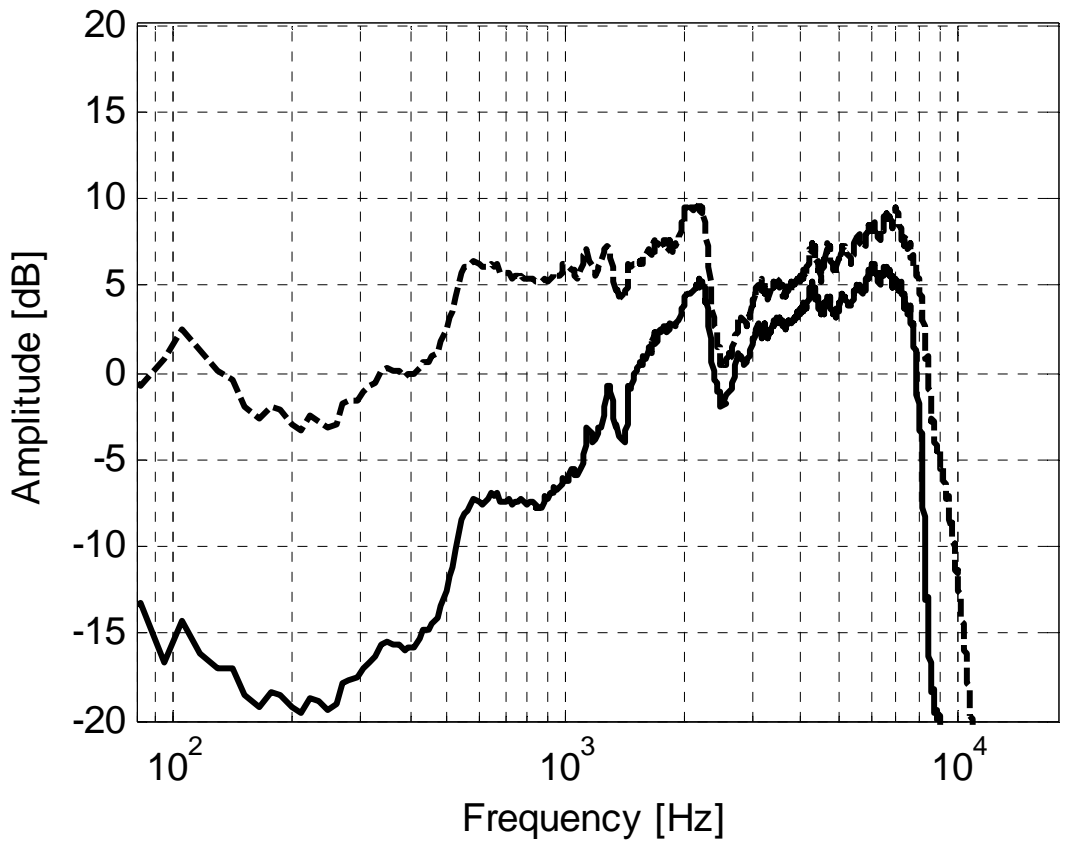


Figure 13

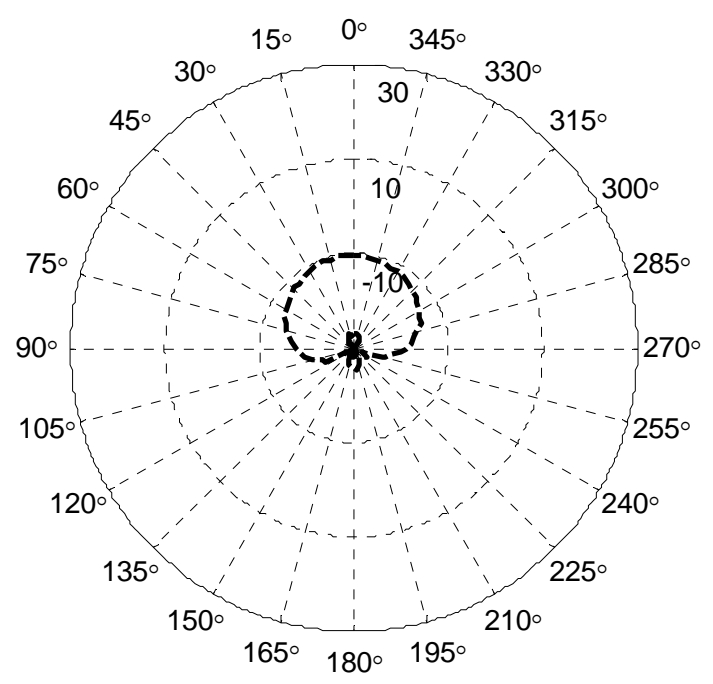

(a)

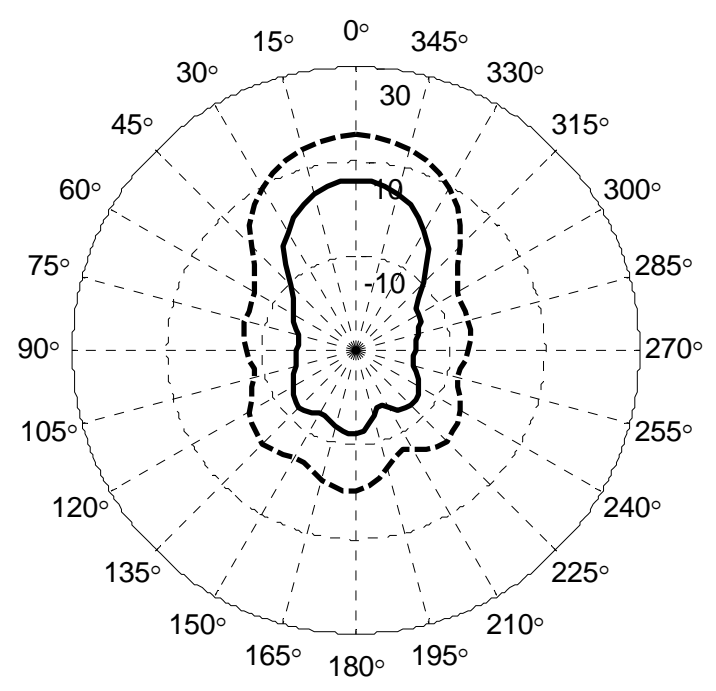

(d)

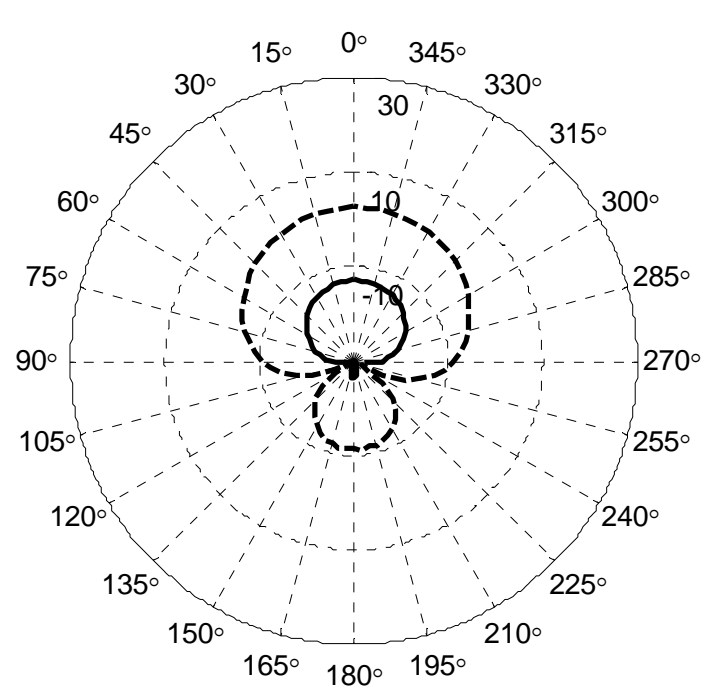

(b)

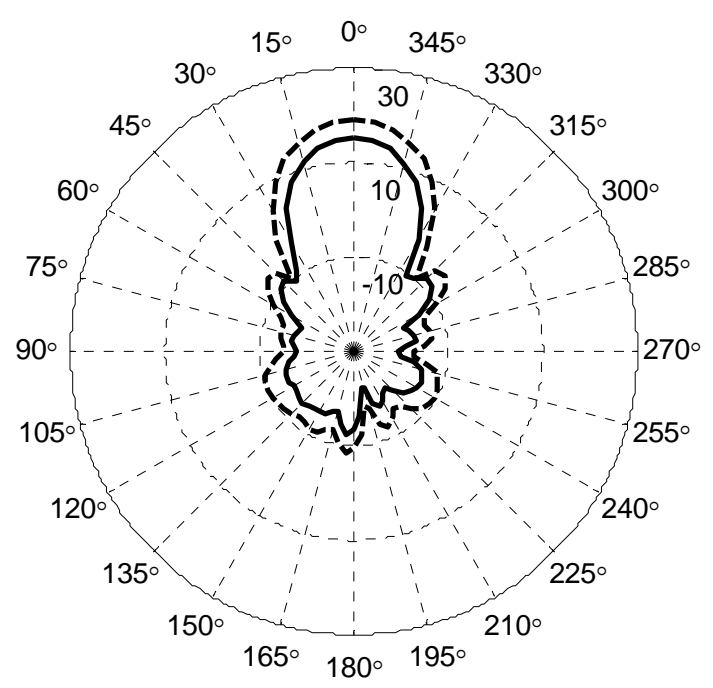

(e)

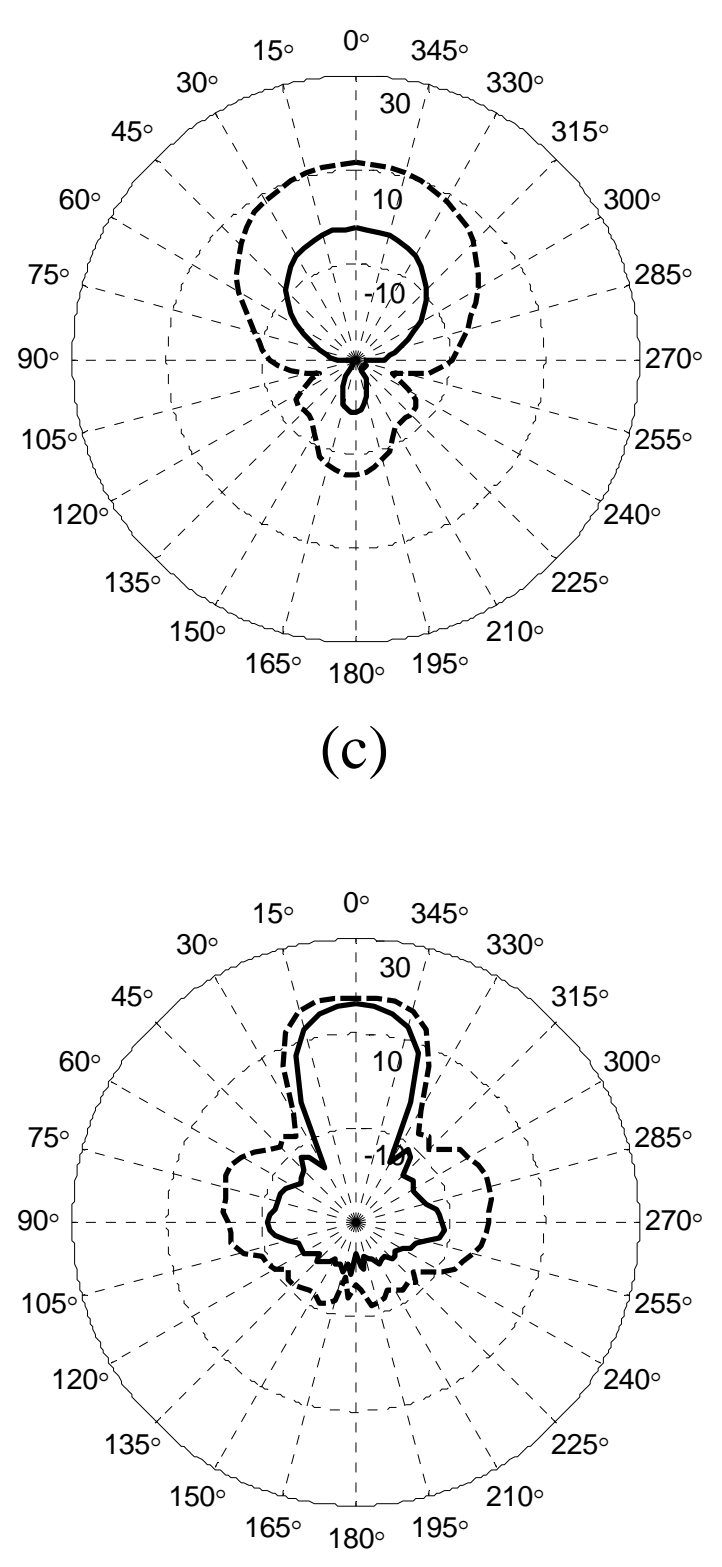

(f) 
1
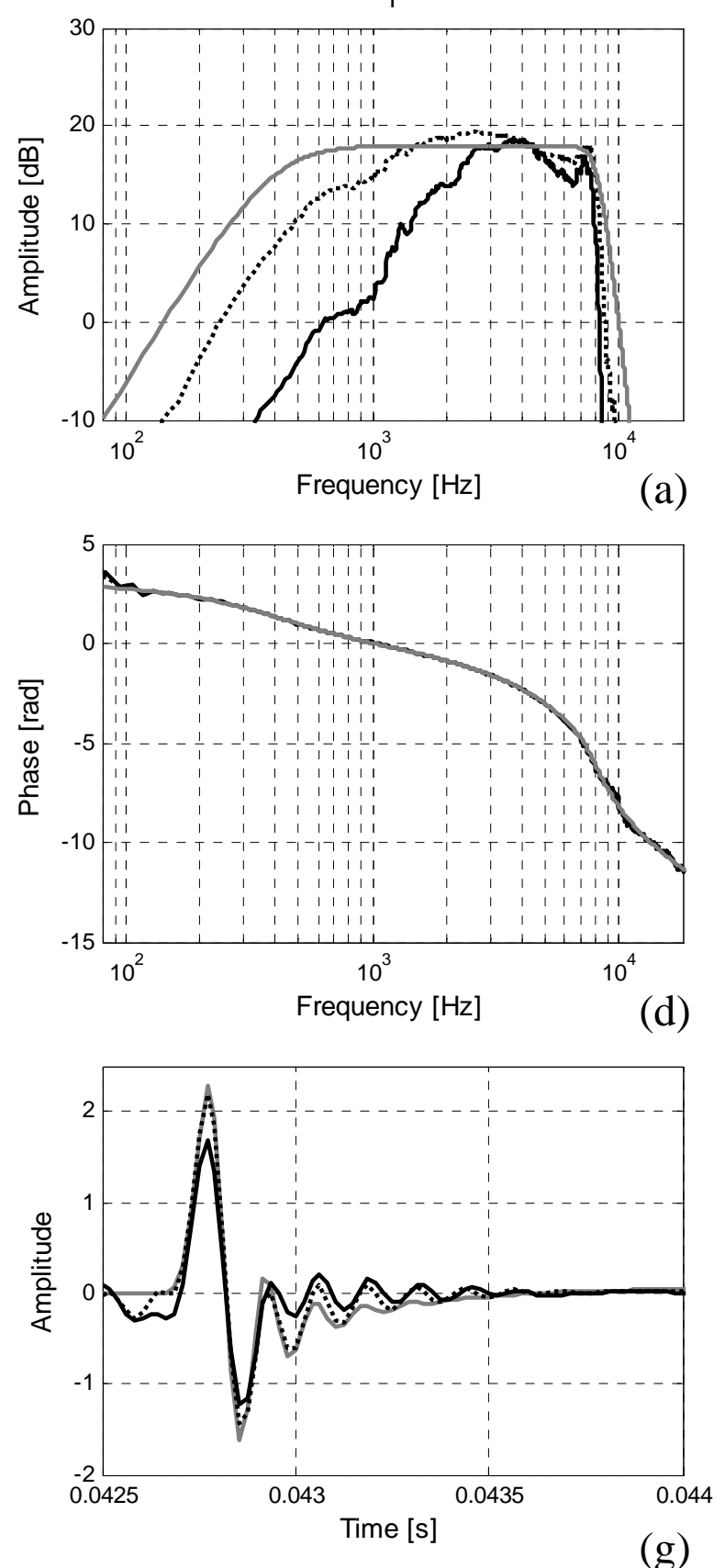

3
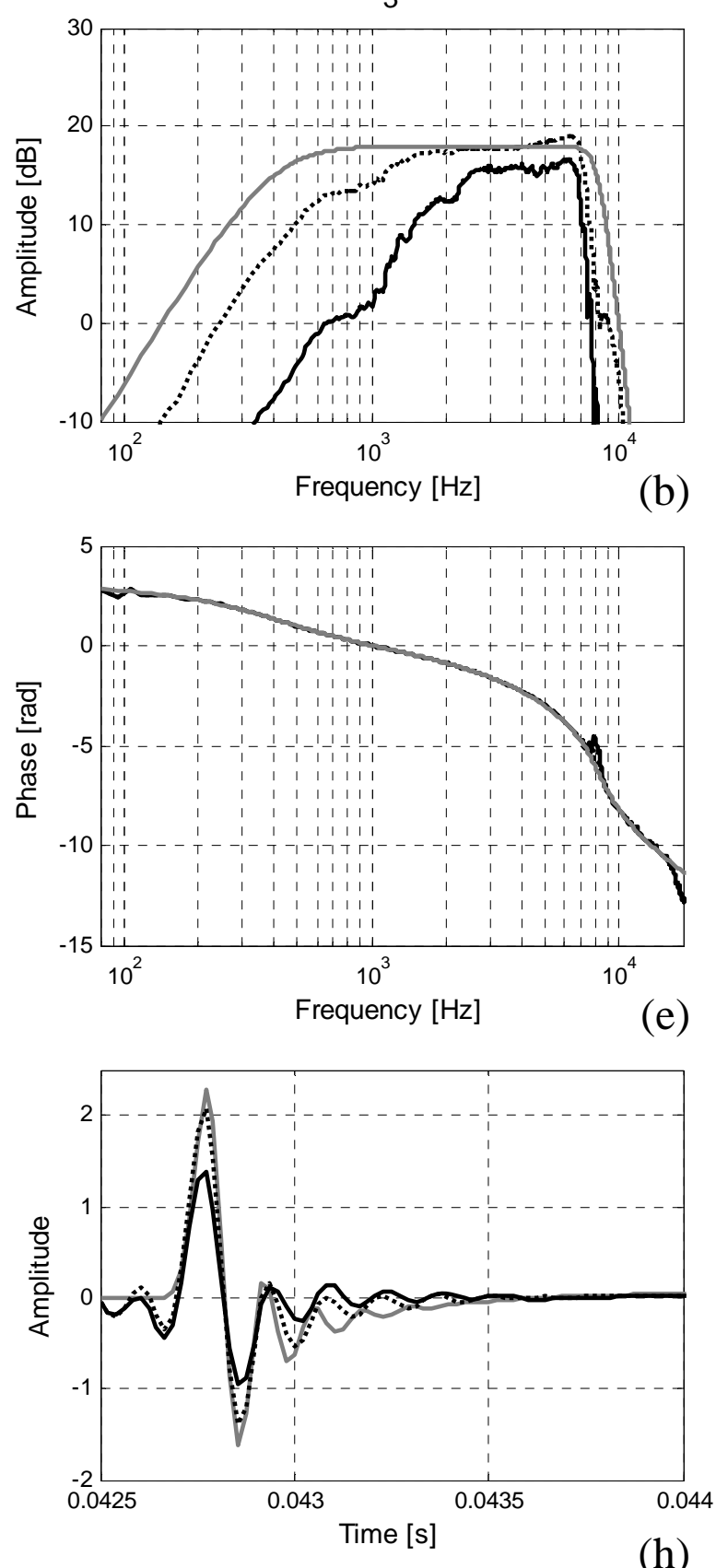

5
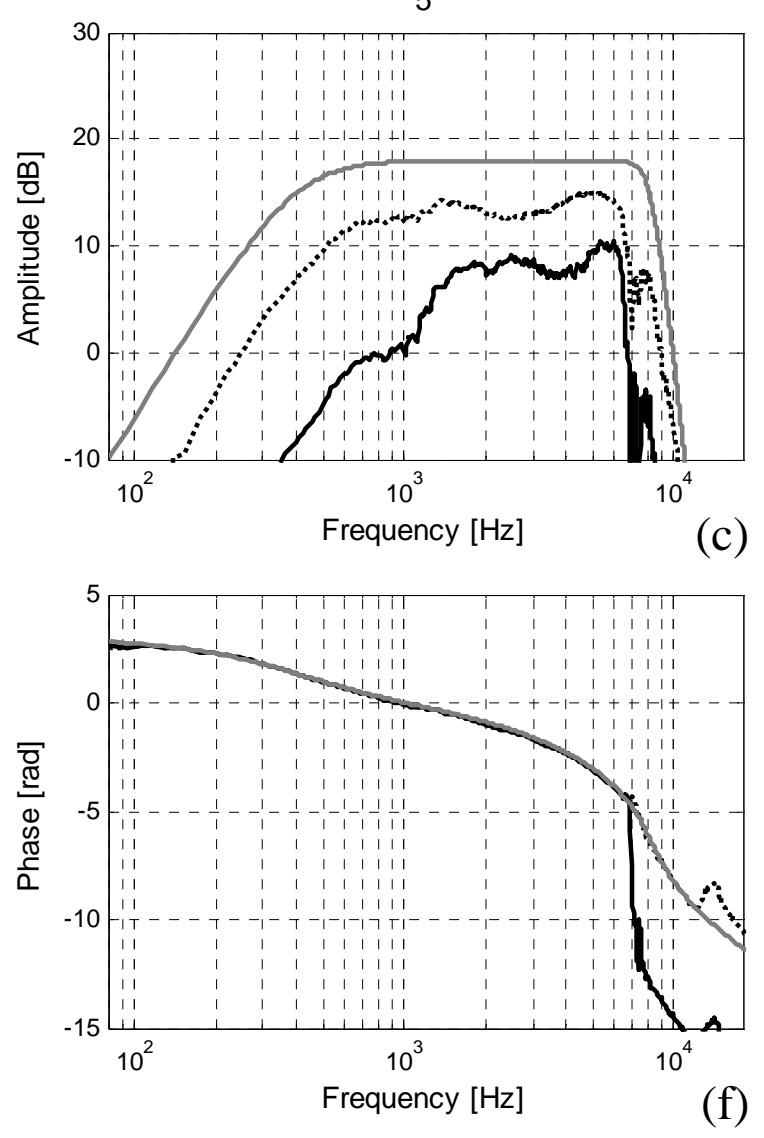

(f)

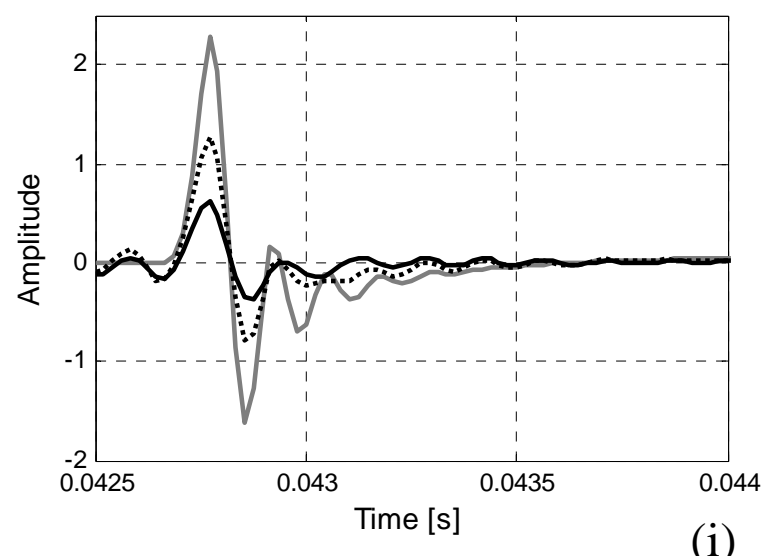

(i) 
Figure 15

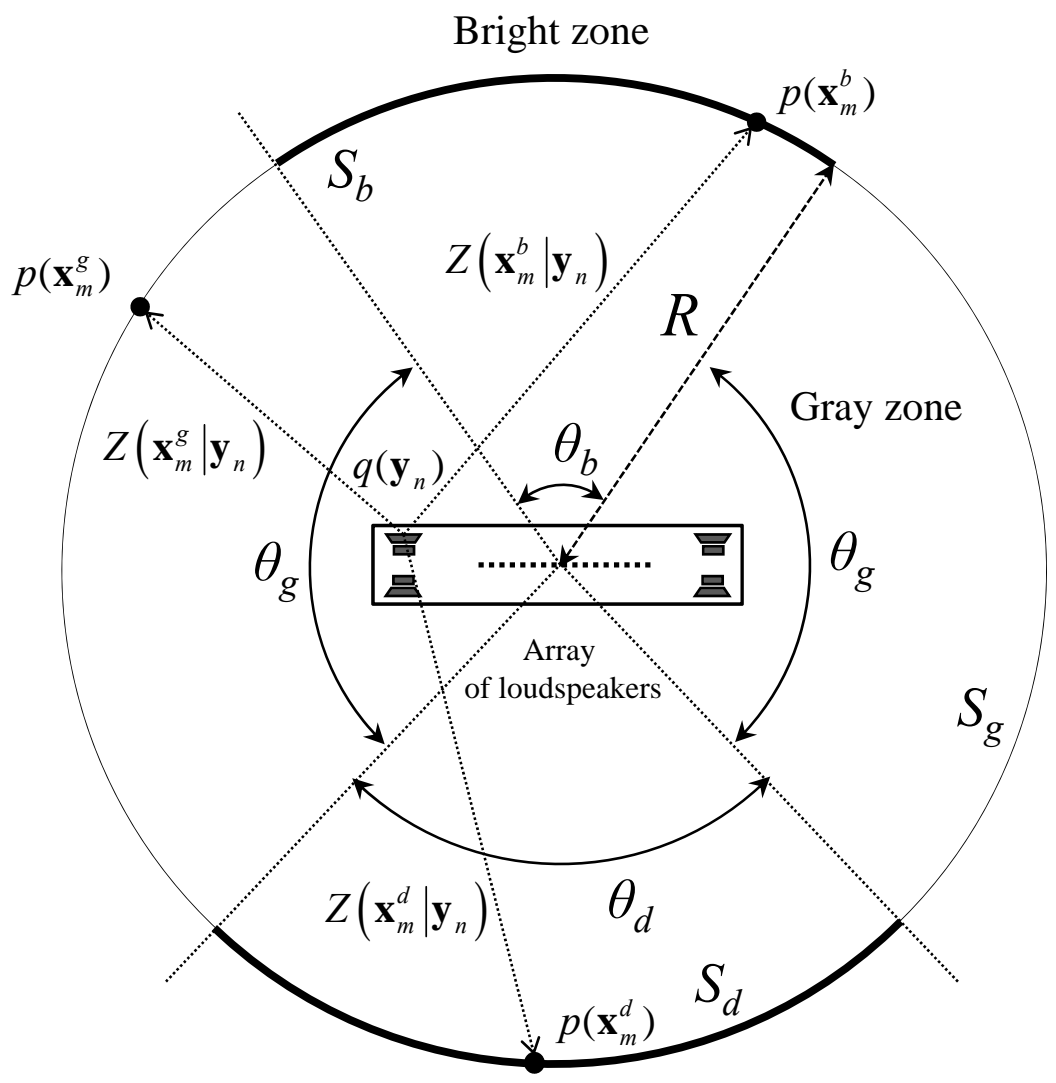

Dark zone 


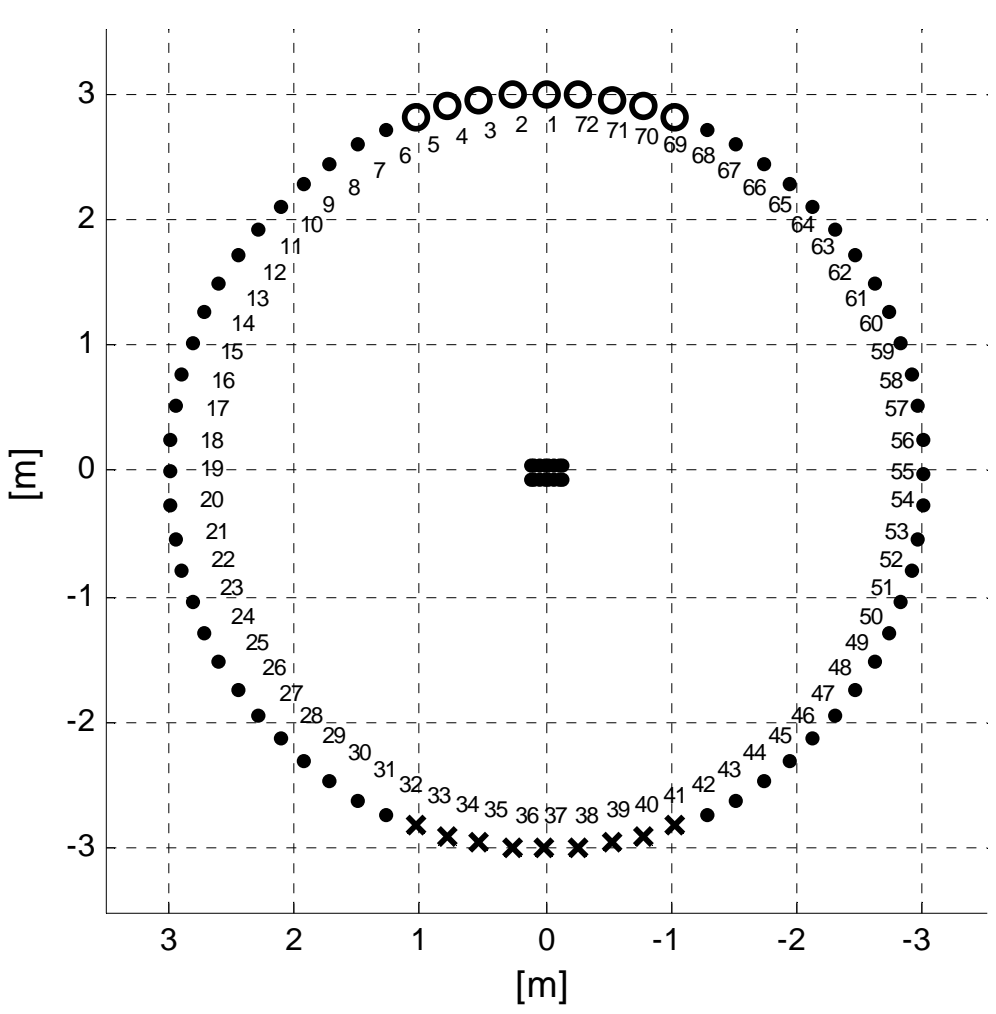

(a)

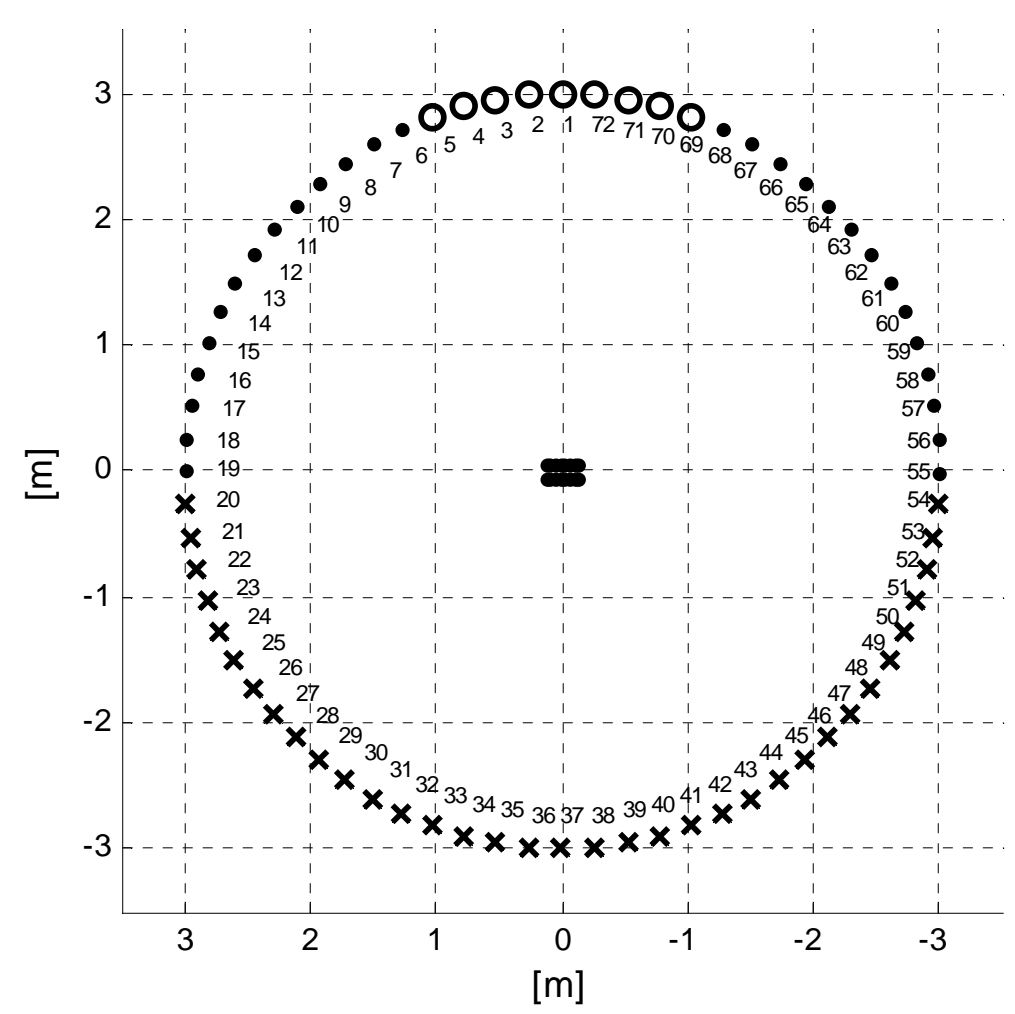

(b) 


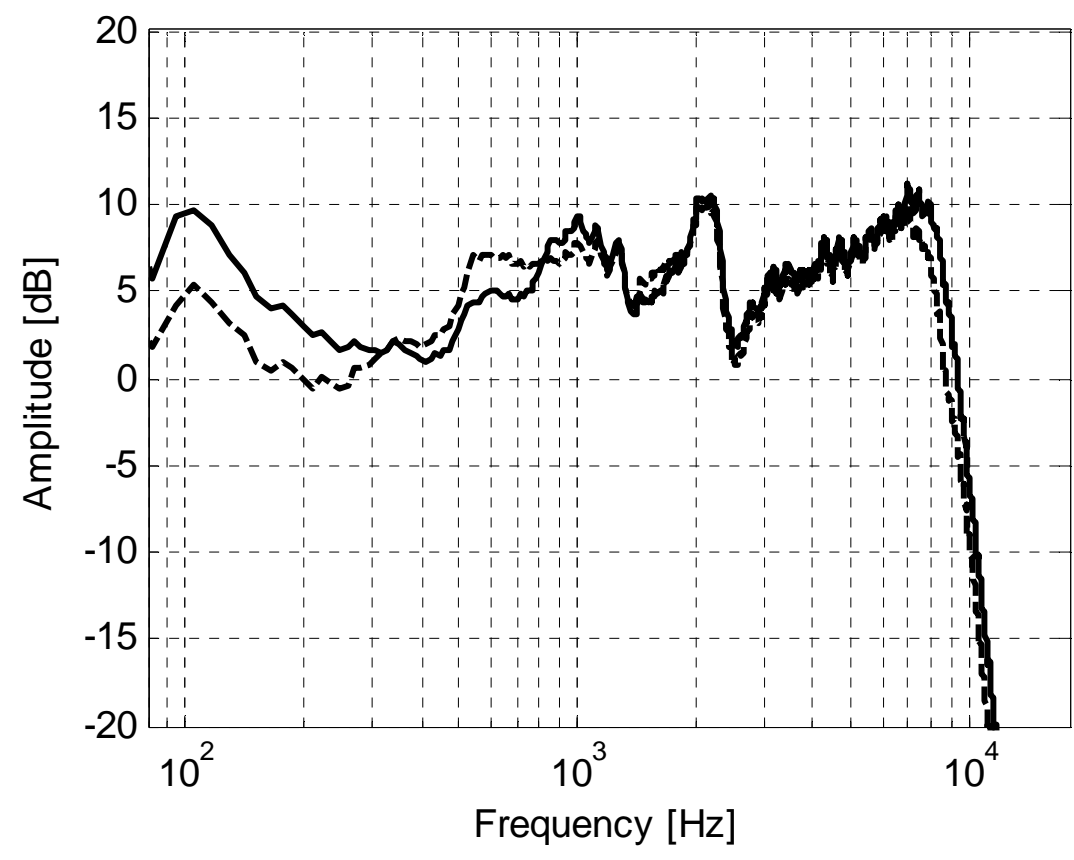

(a)

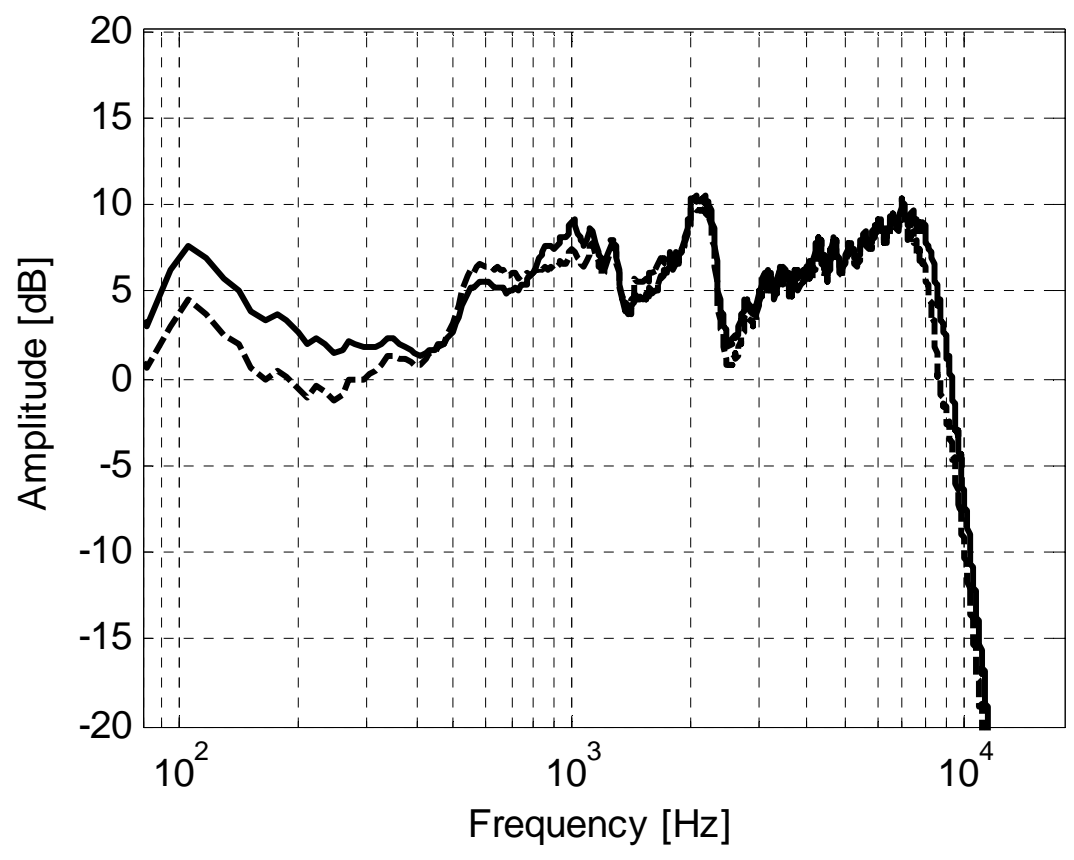

(b) 


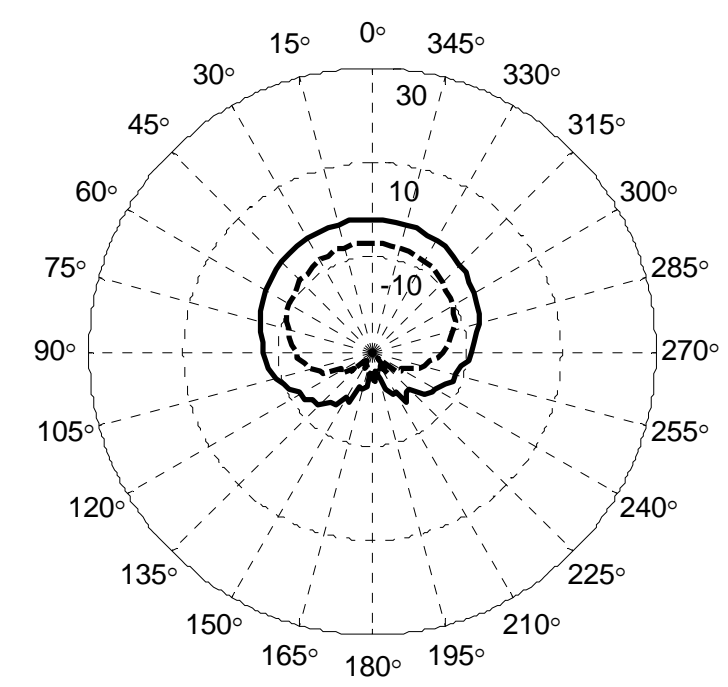

(a)

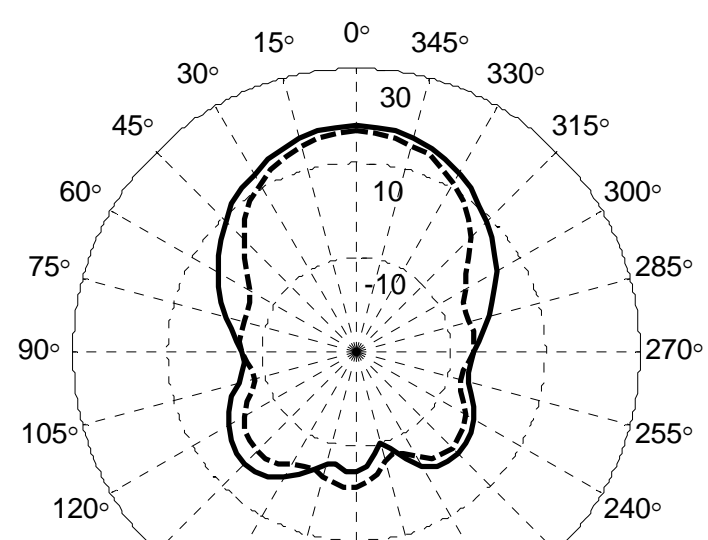

$135^{\circ}$

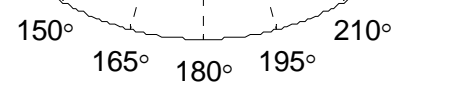

(d)

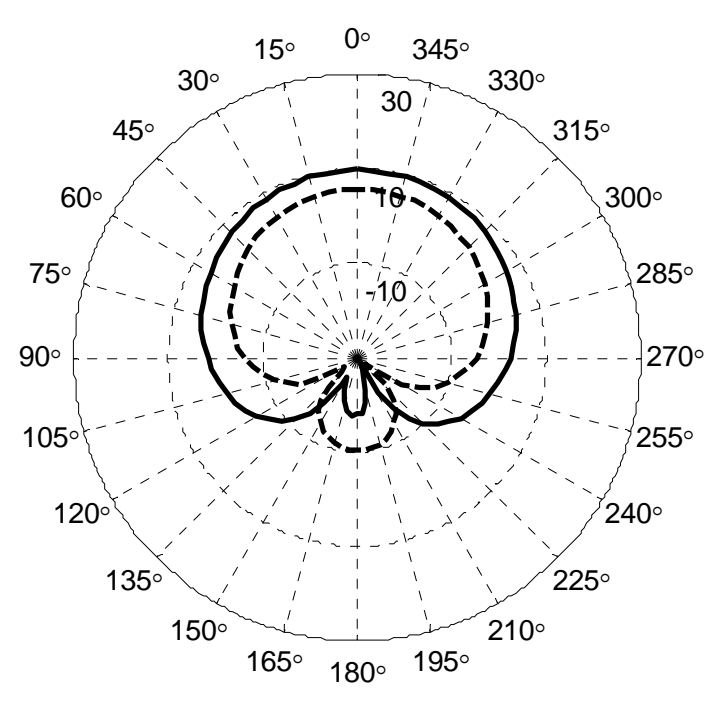

(b)

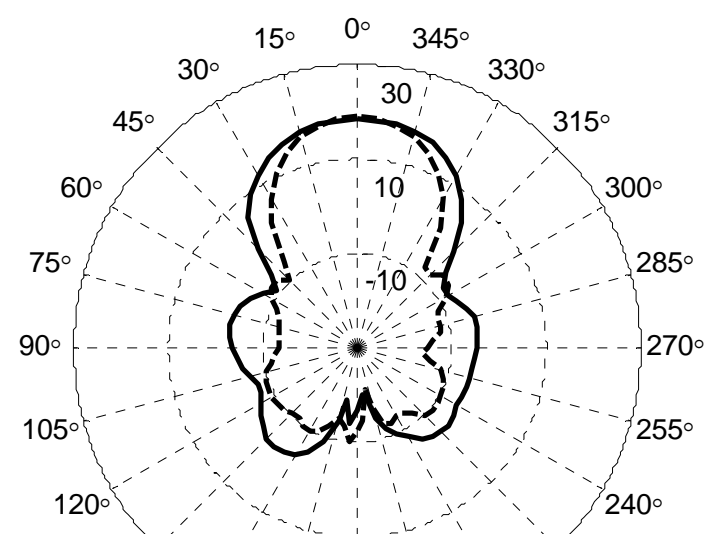

$135^{\circ}$

$225^{\circ}$

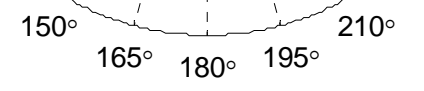

(e)

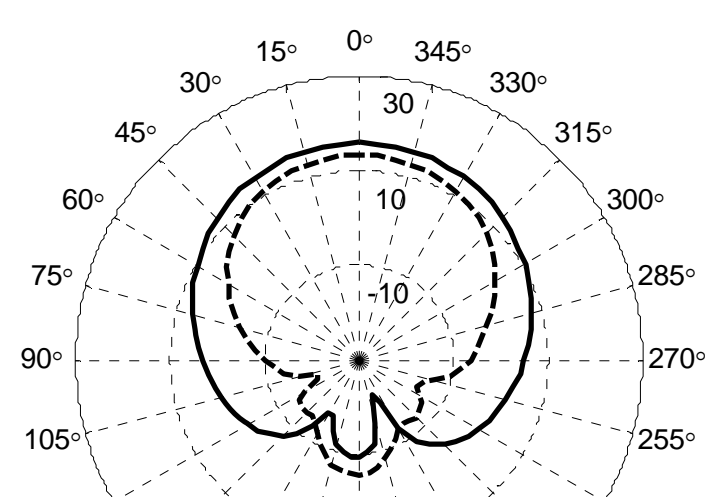

$120^{\circ}$

$240^{\circ}$

$135^{\circ}$

$225^{\circ}$

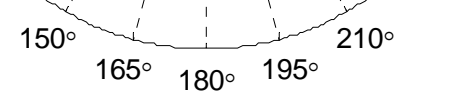

(c)

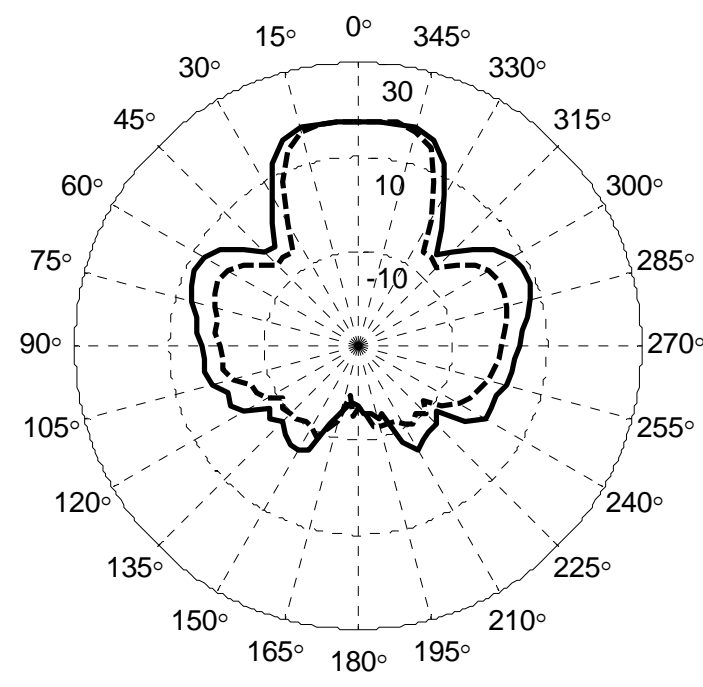

(f) 

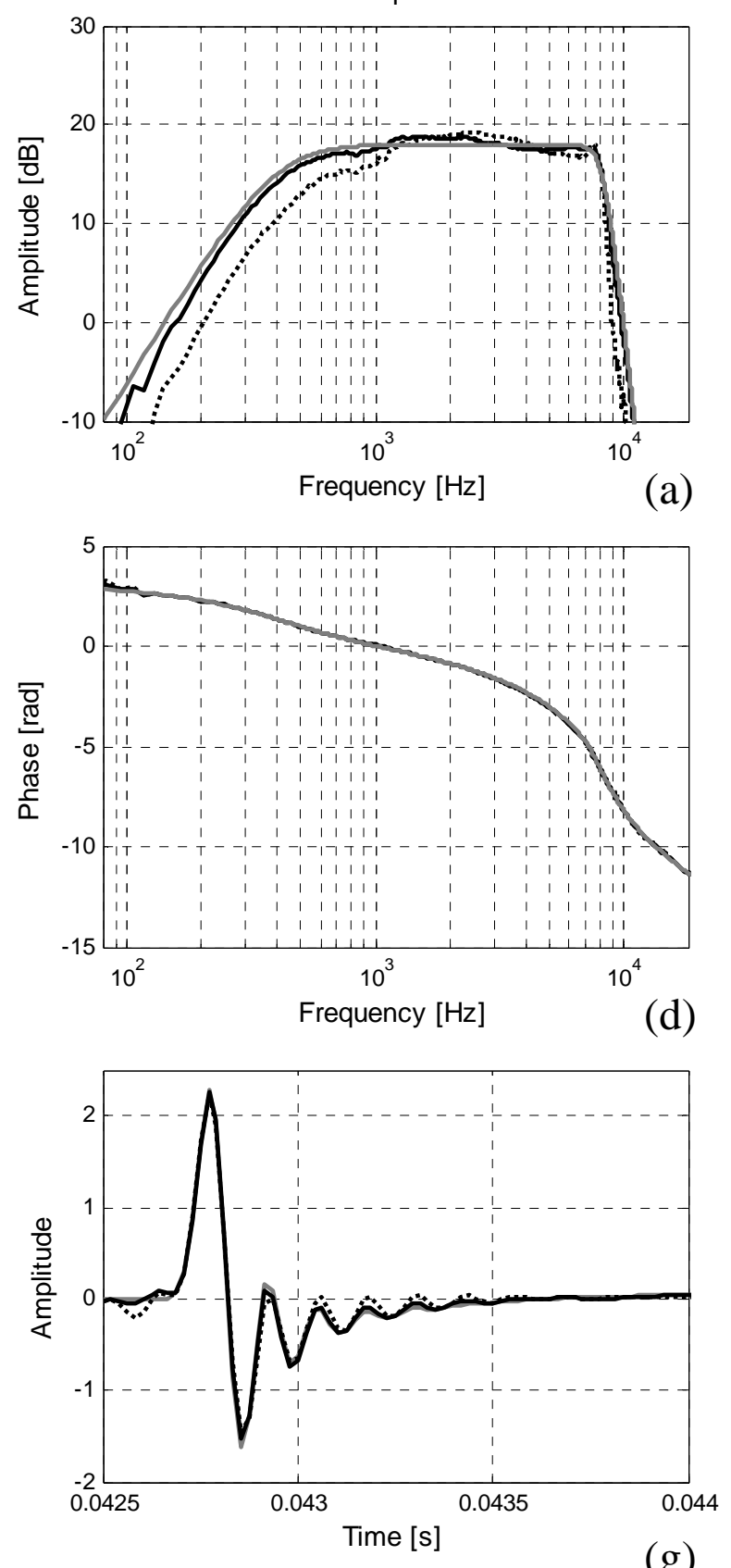
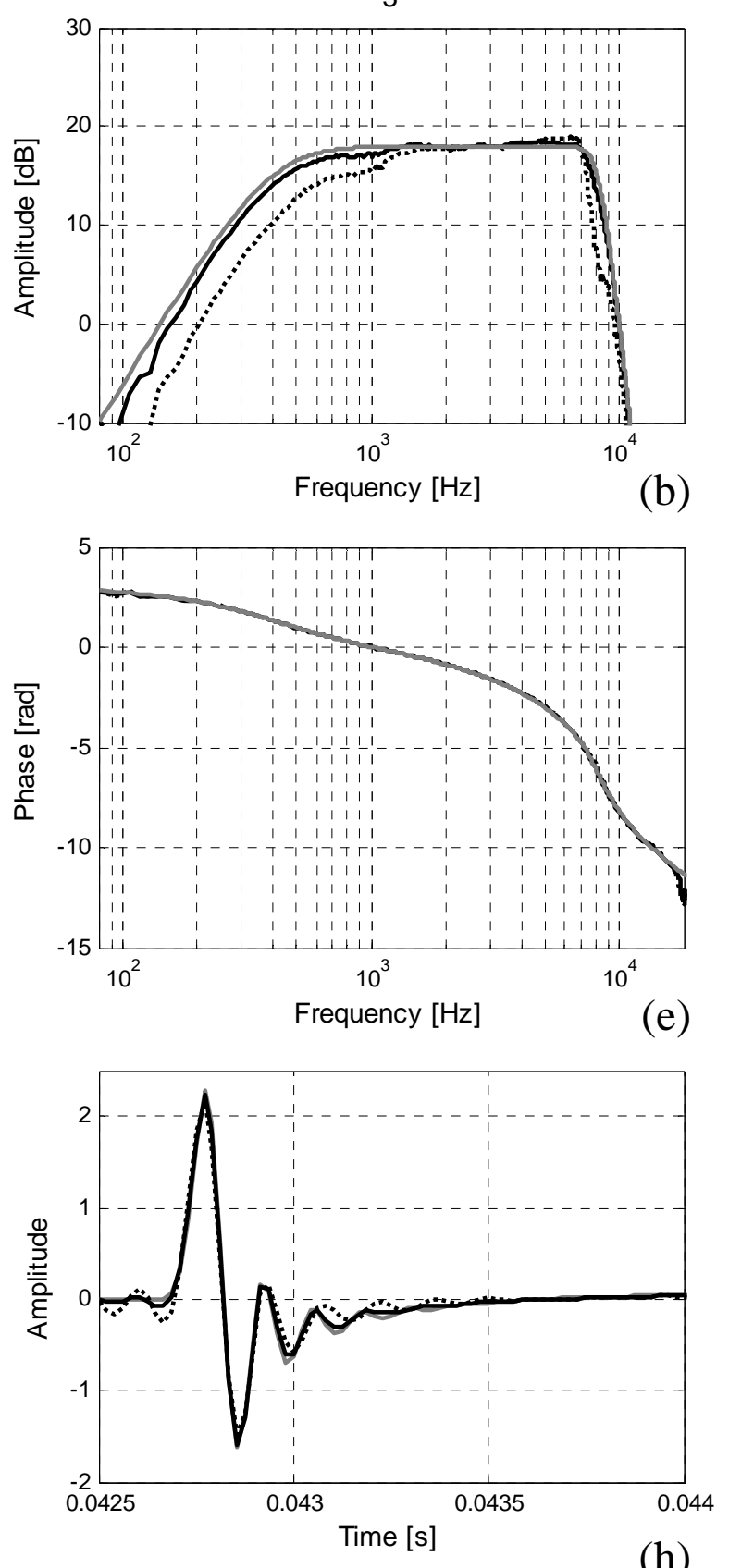

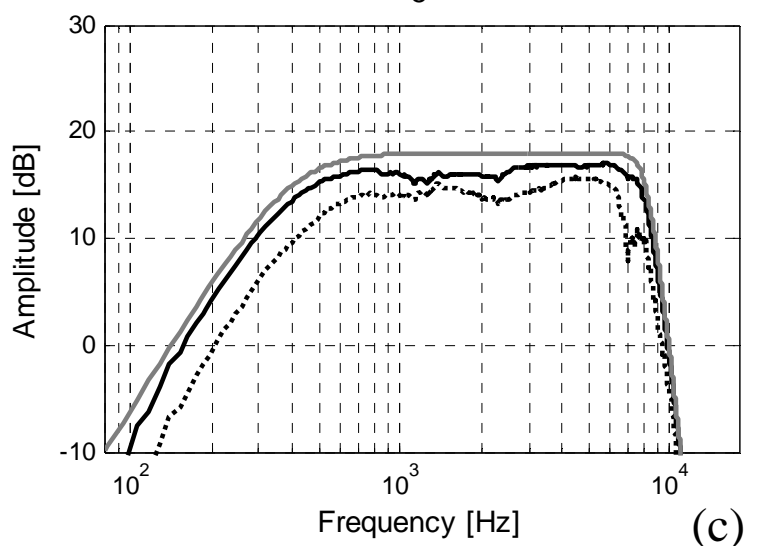

(c)

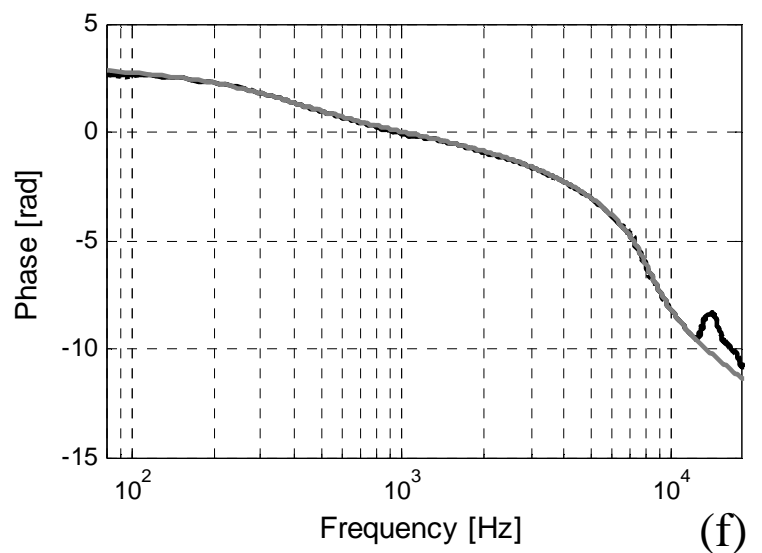

(f)

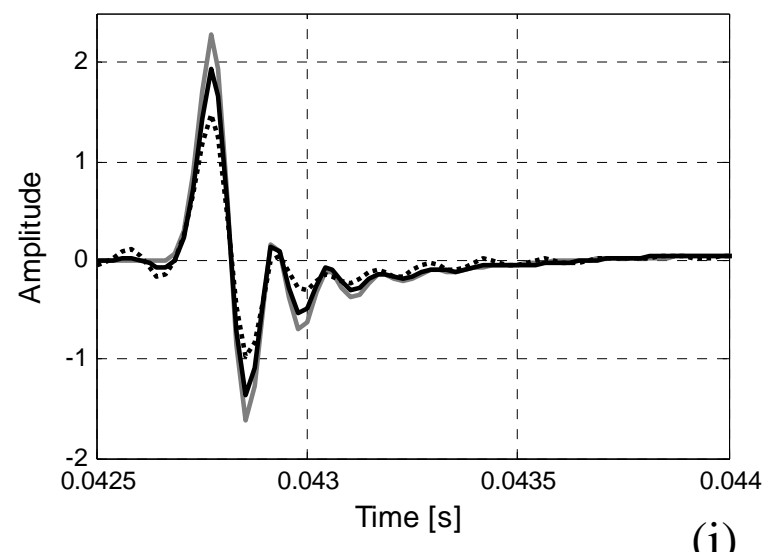




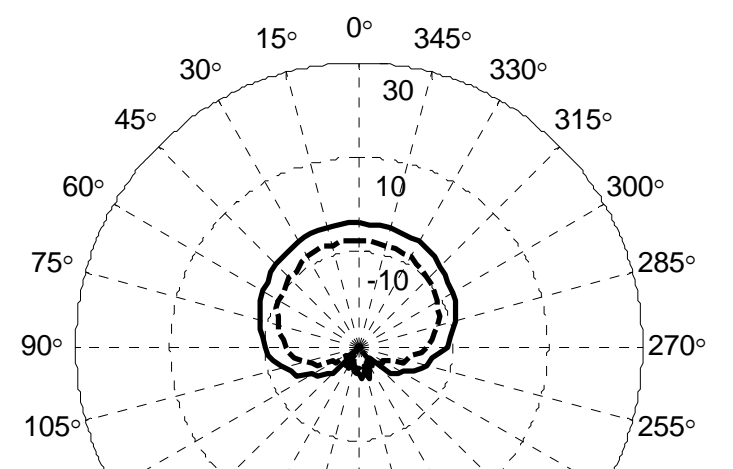

$120^{\circ}$

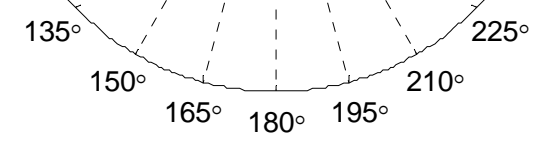

(a)

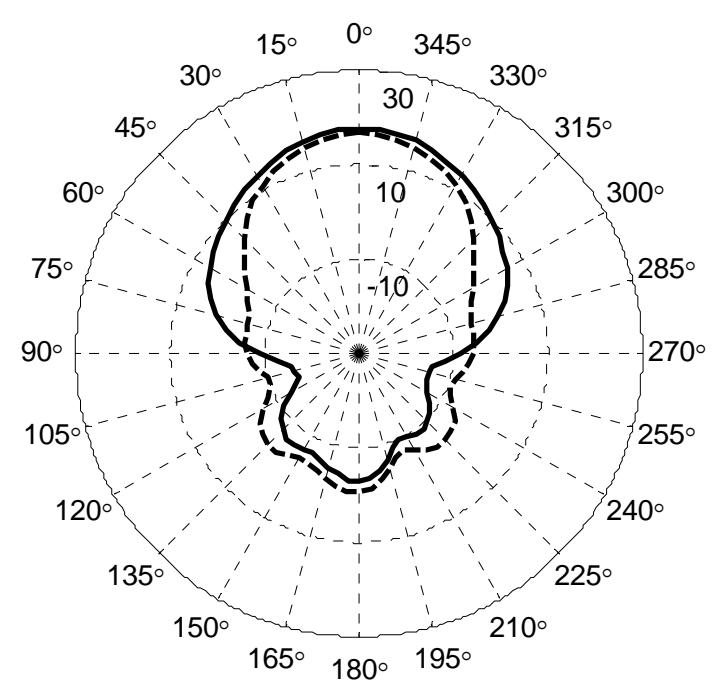

(d)

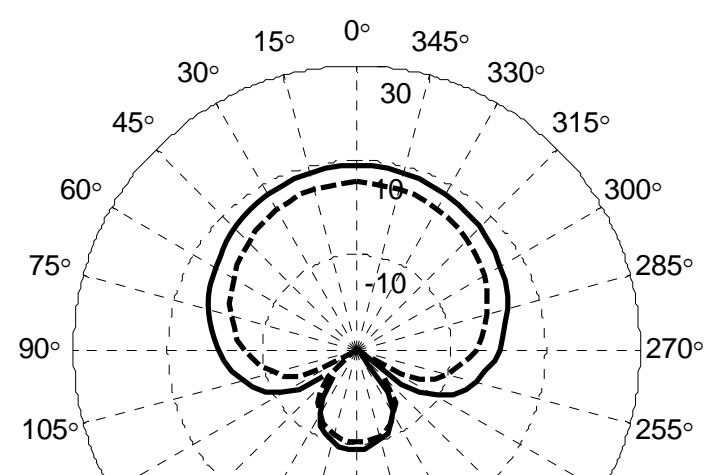

$120^{\circ}$

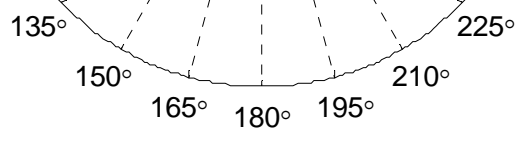

(b)
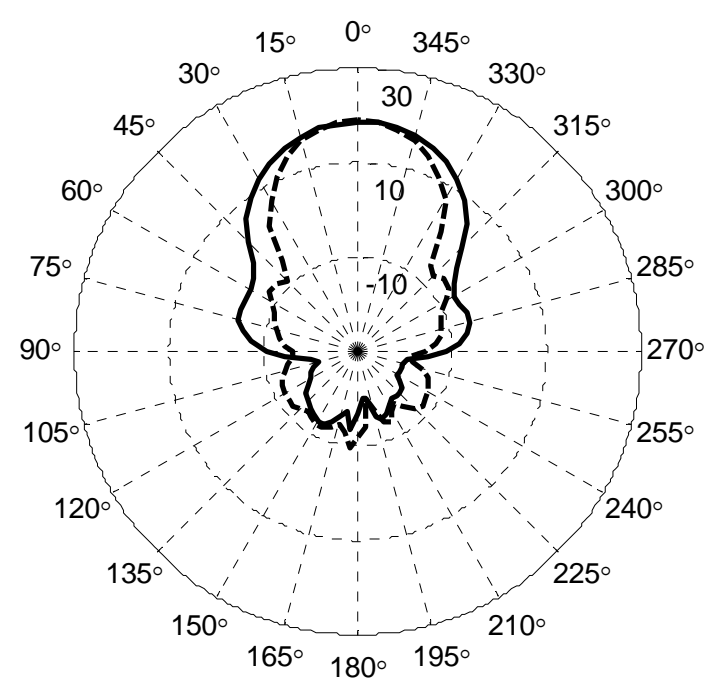

(e)

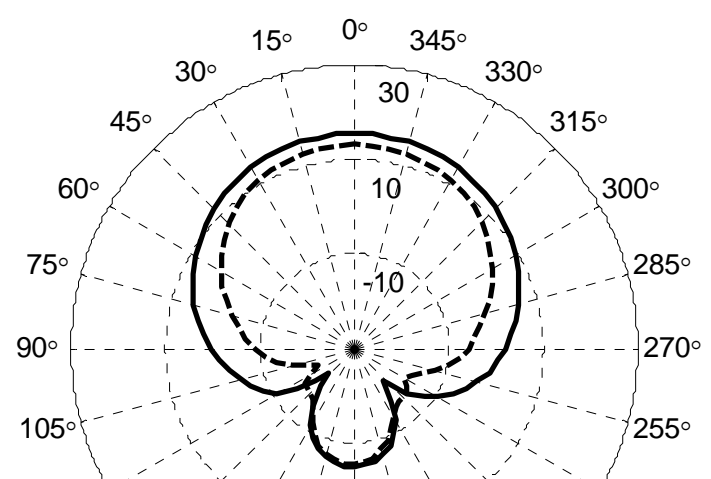

$120^{\circ}$

$240^{\circ}$

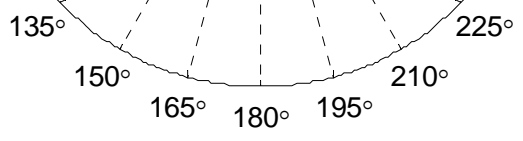

(c)

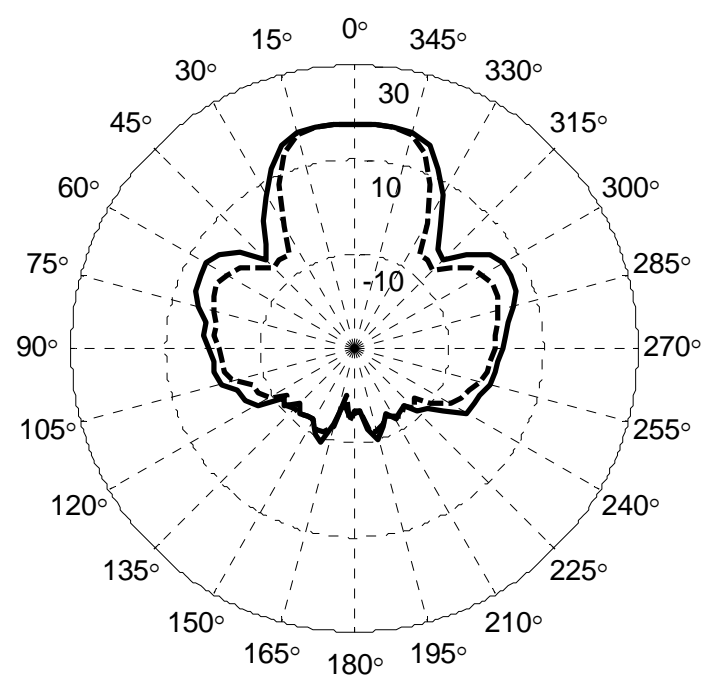

(f) 
1

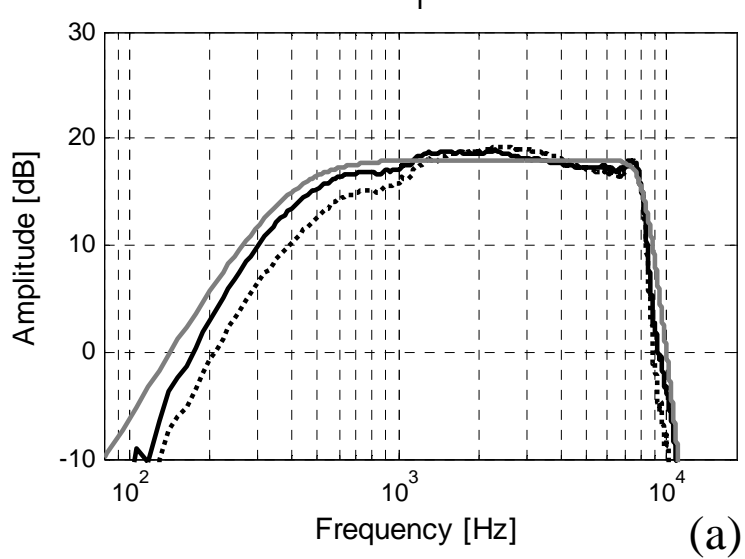

(a)
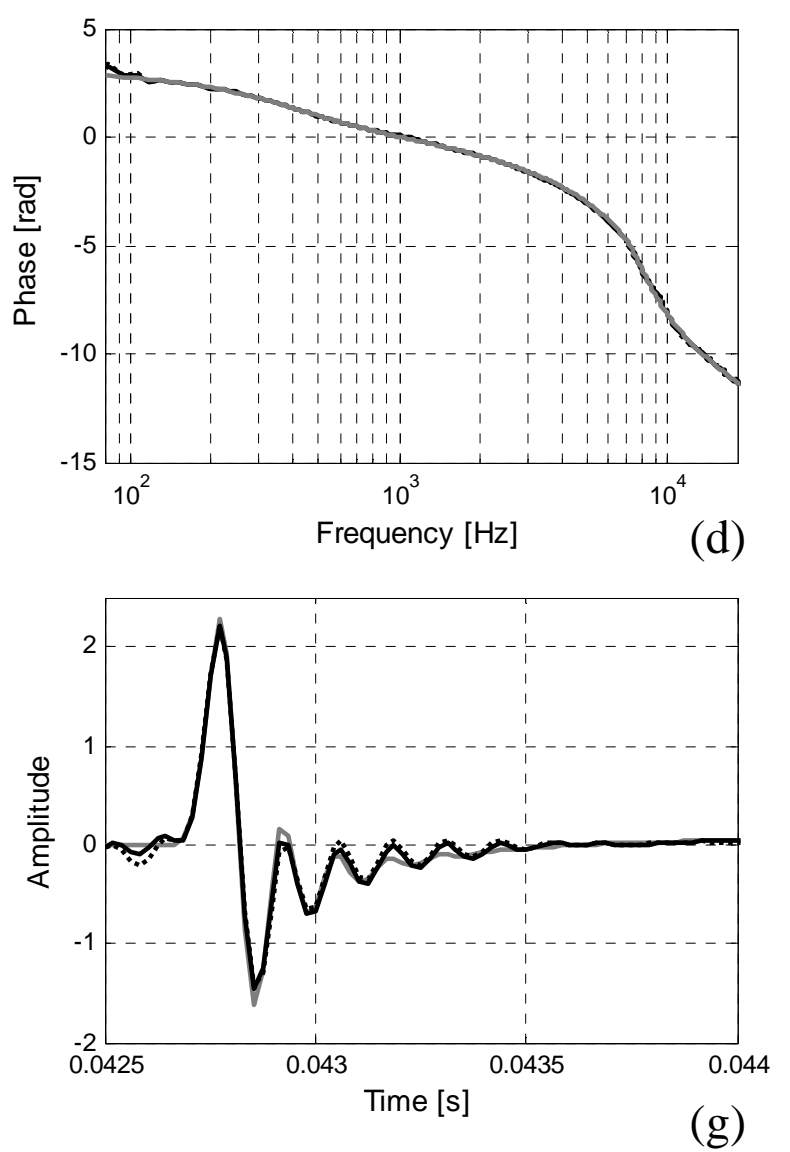

3
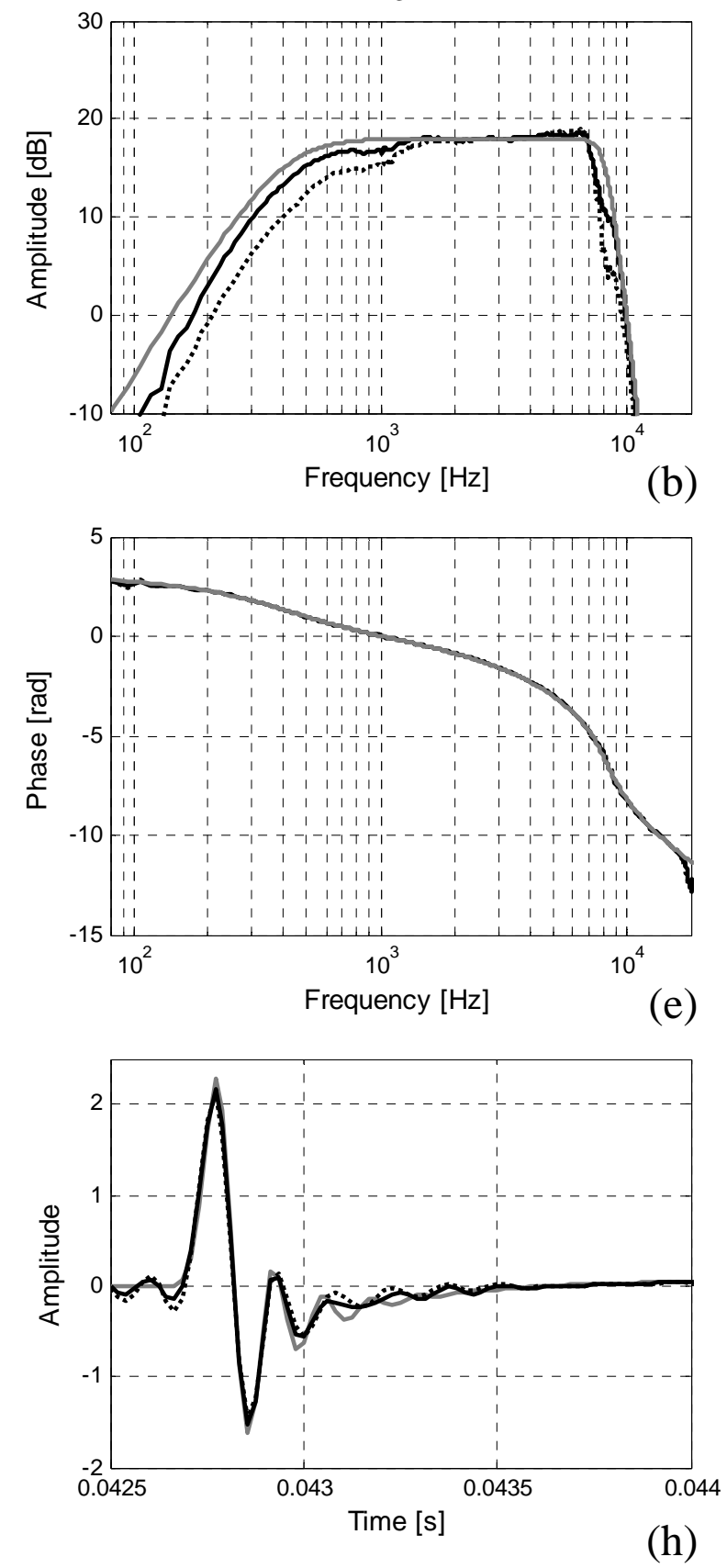

5
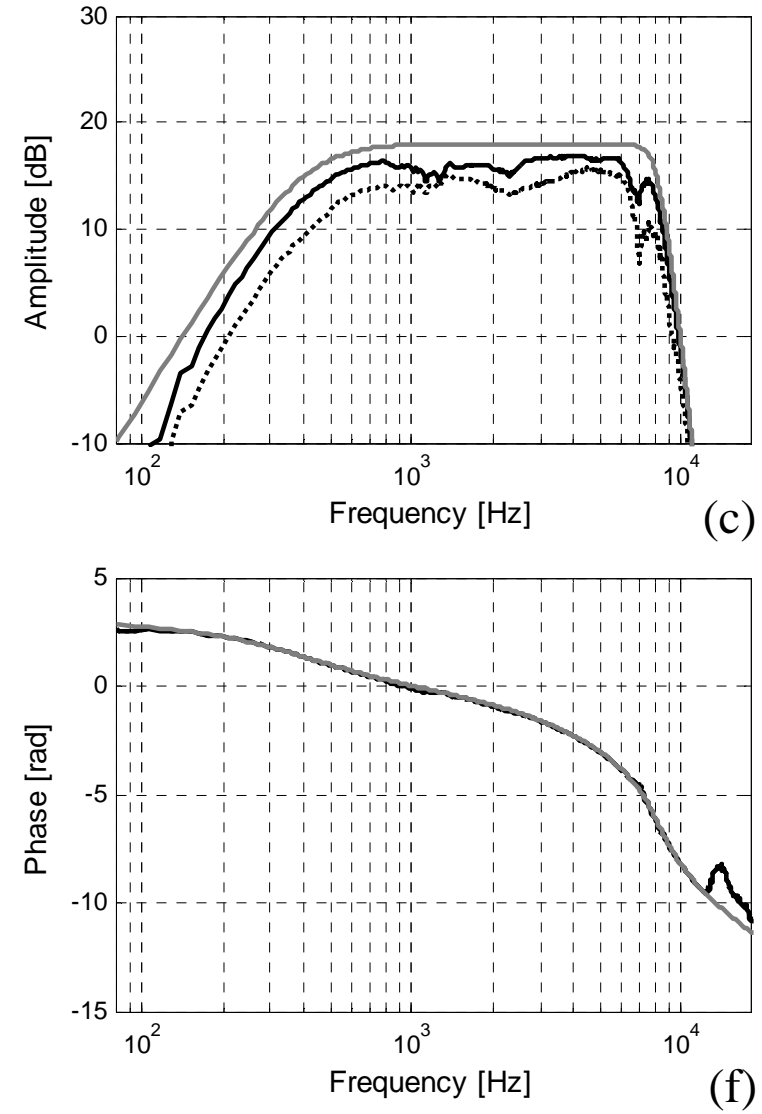

(f)

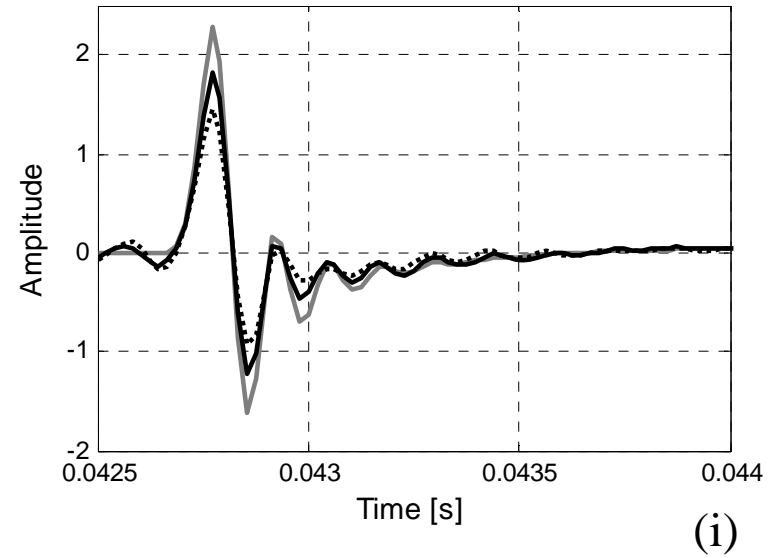



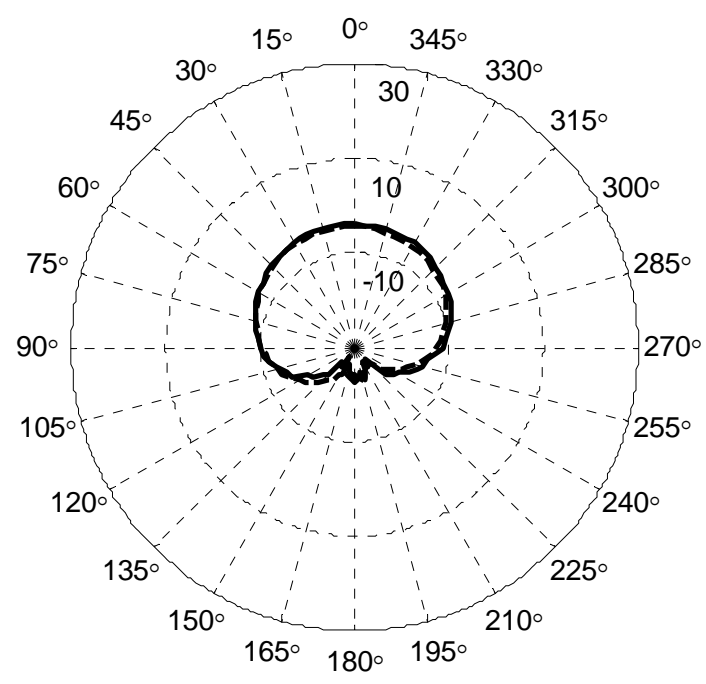

(a)

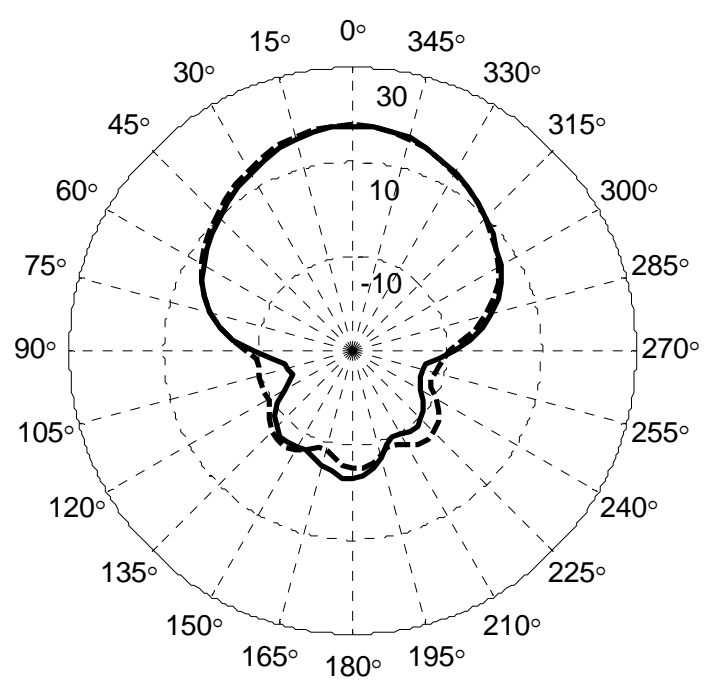

(d)

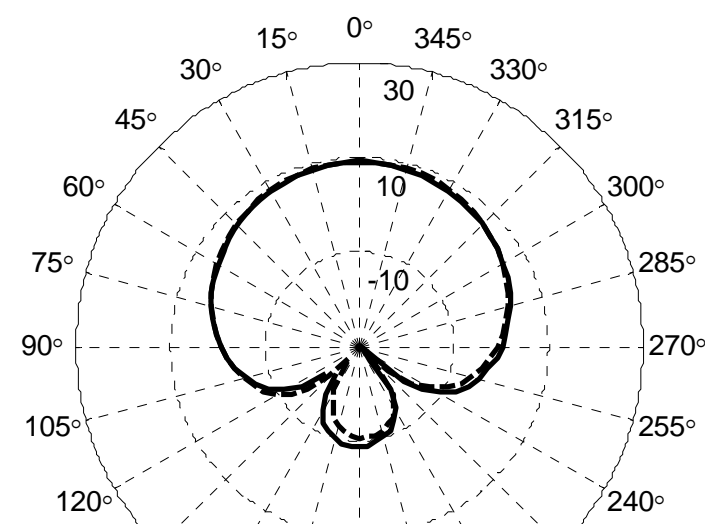

$135^{\circ}$

$225^{\circ}$

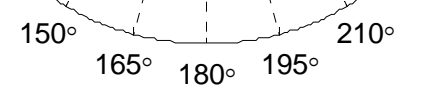

(b)

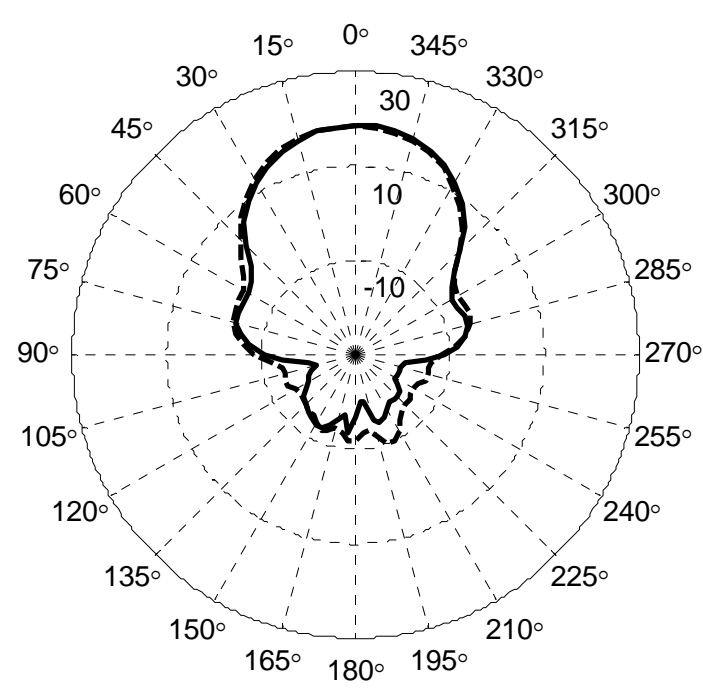

(e)
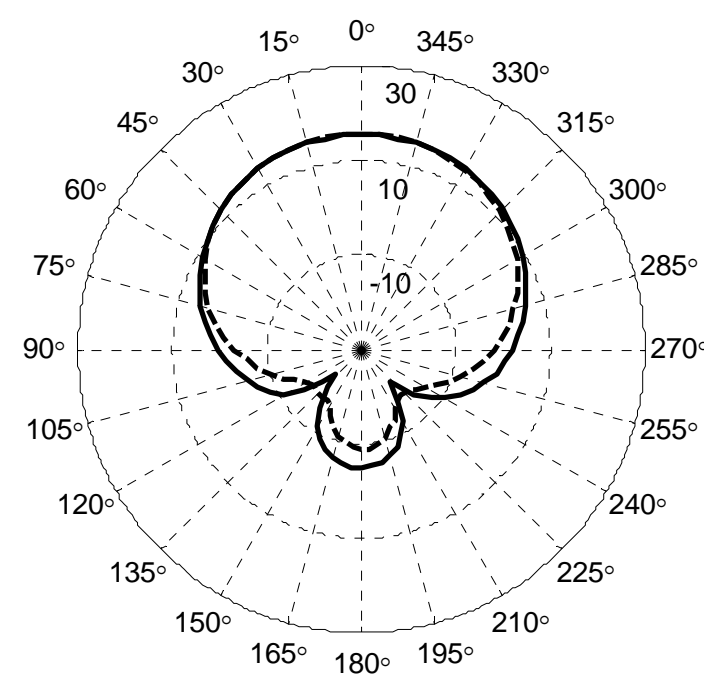

(c)

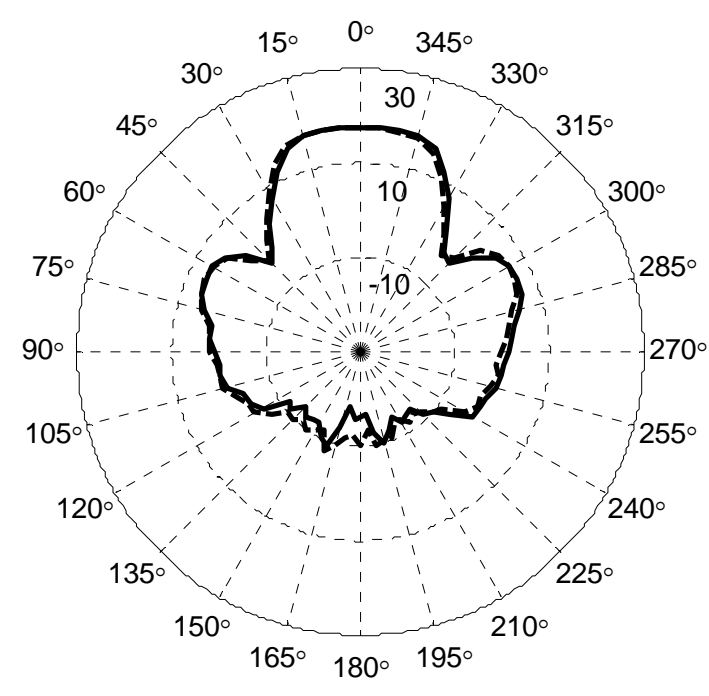

(f) 
Figure 23

1
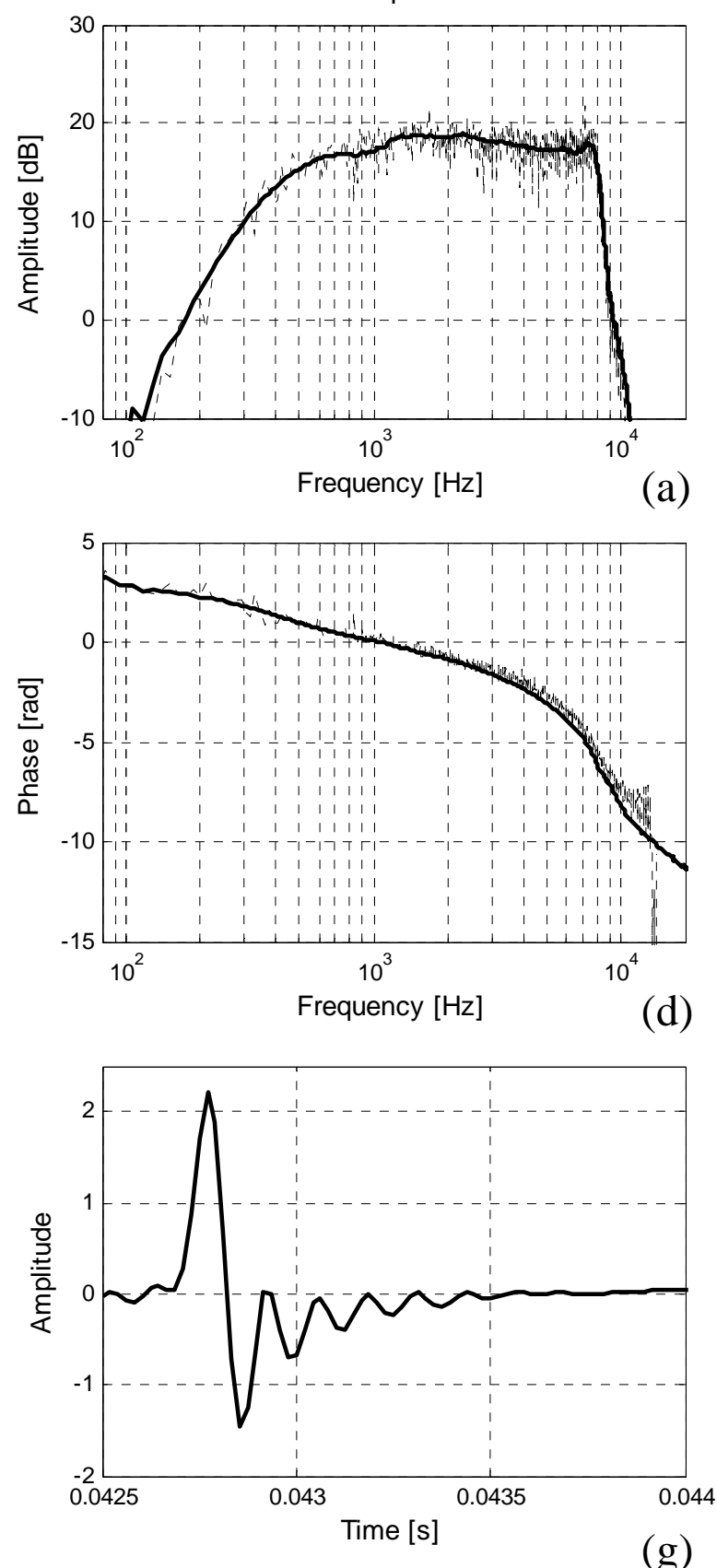

3
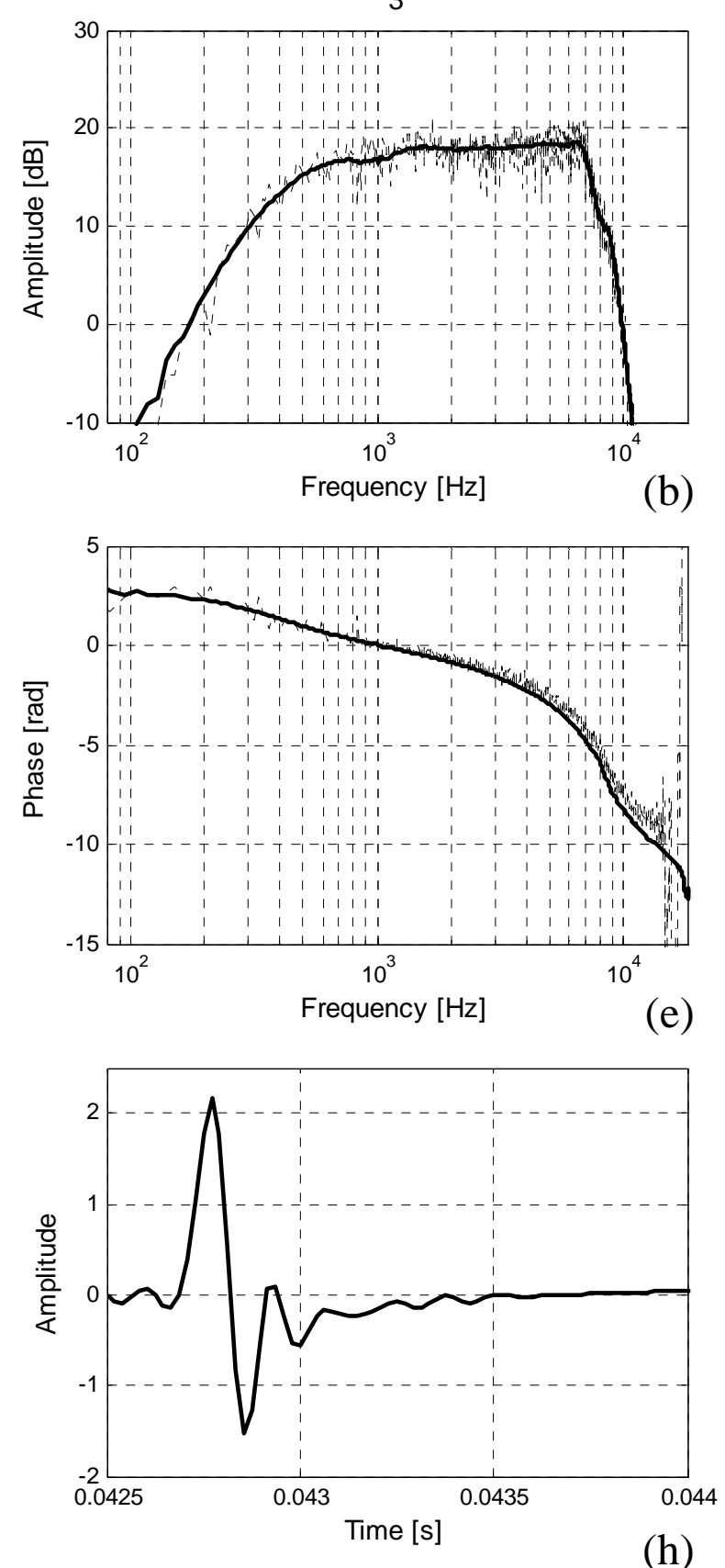

5
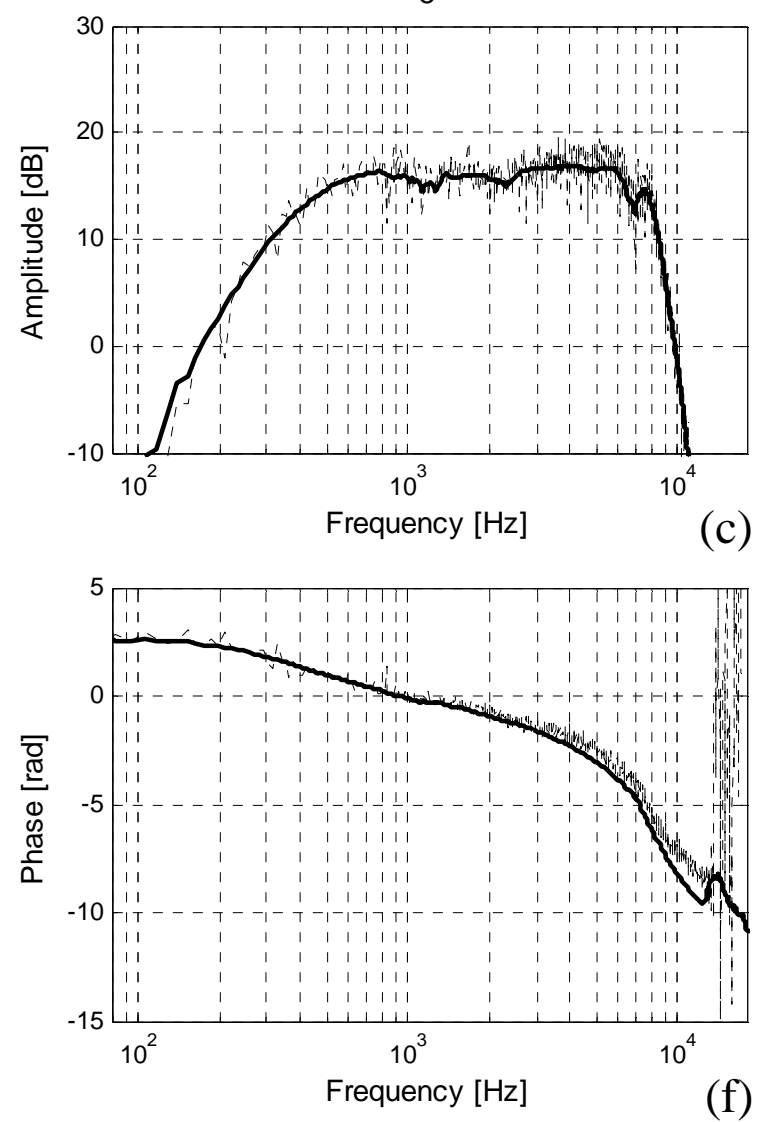

(f)

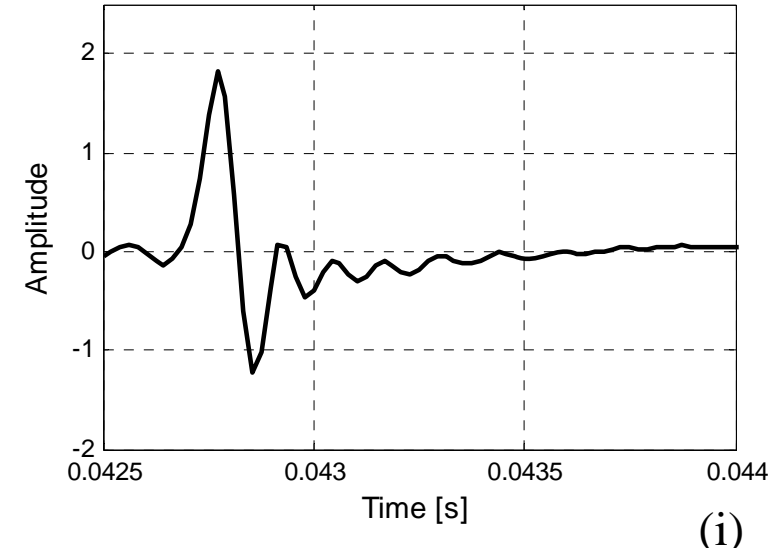




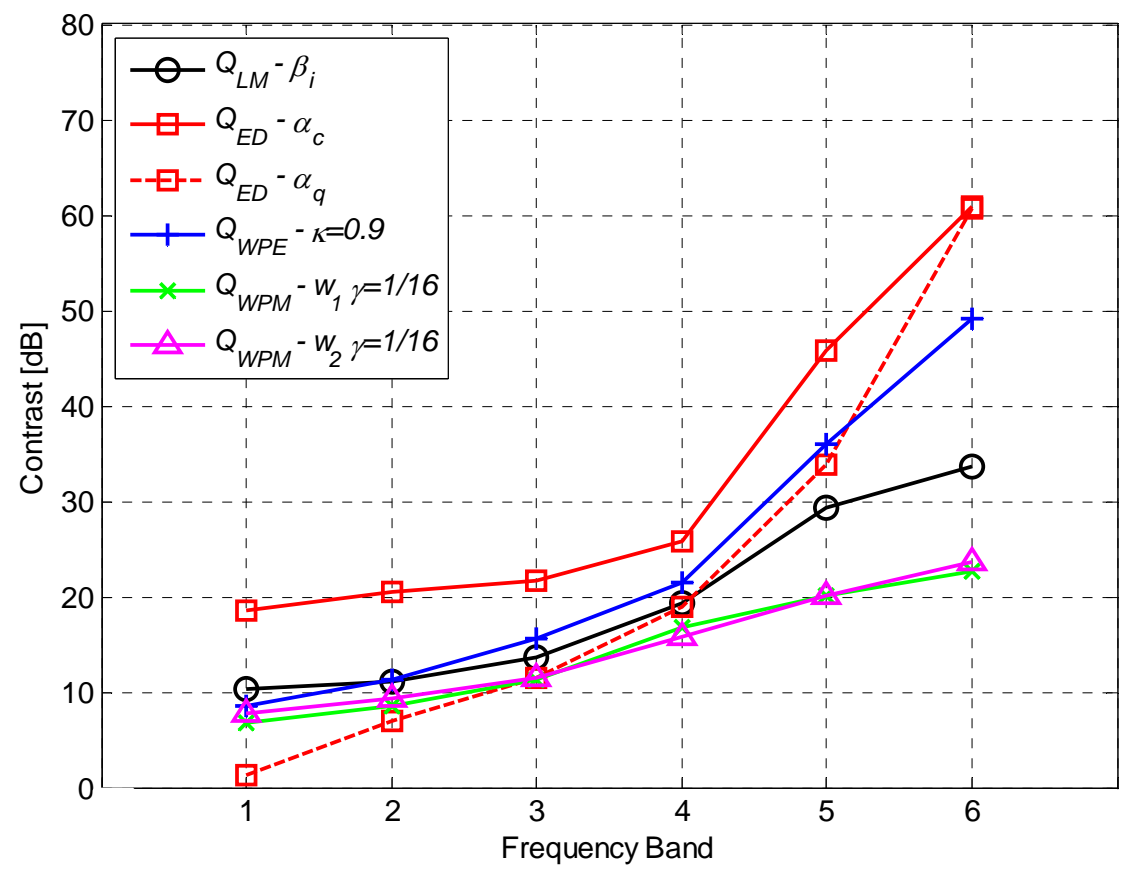

(a)

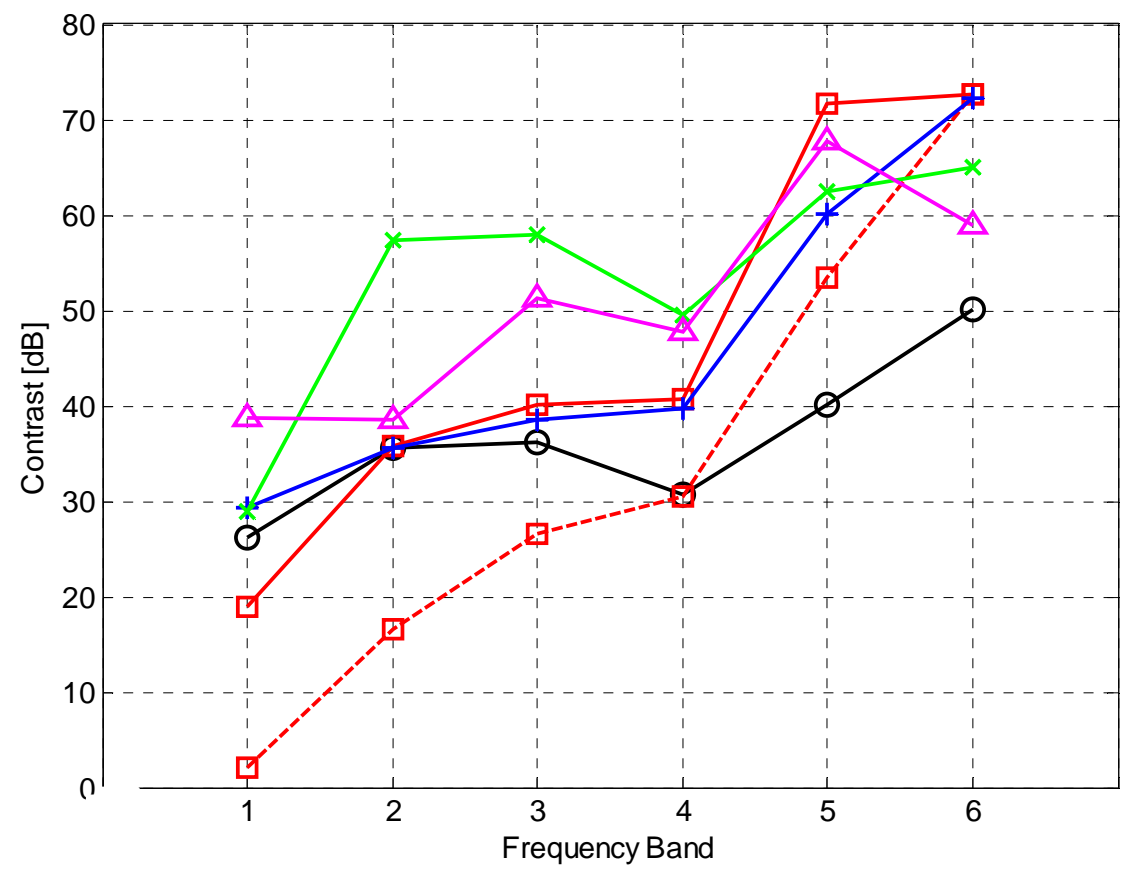

(b) 
Figure 25

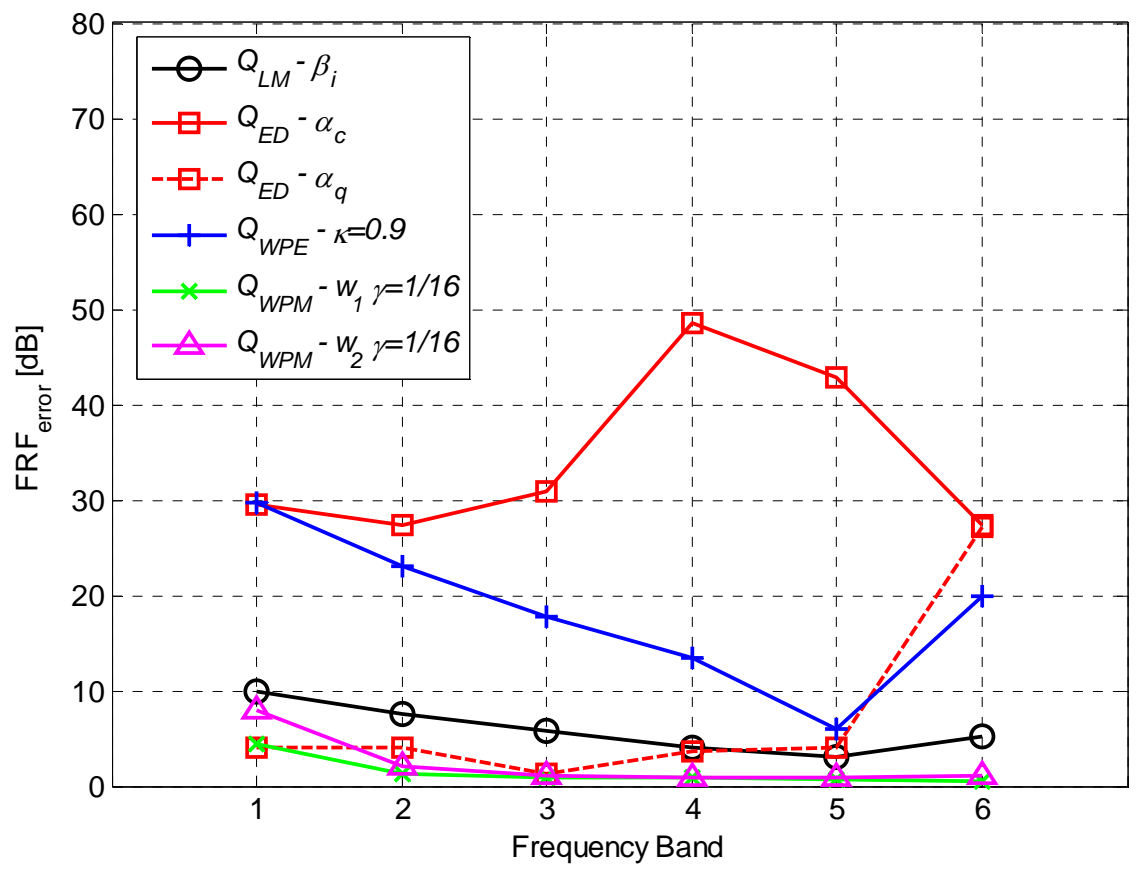


Figure 26

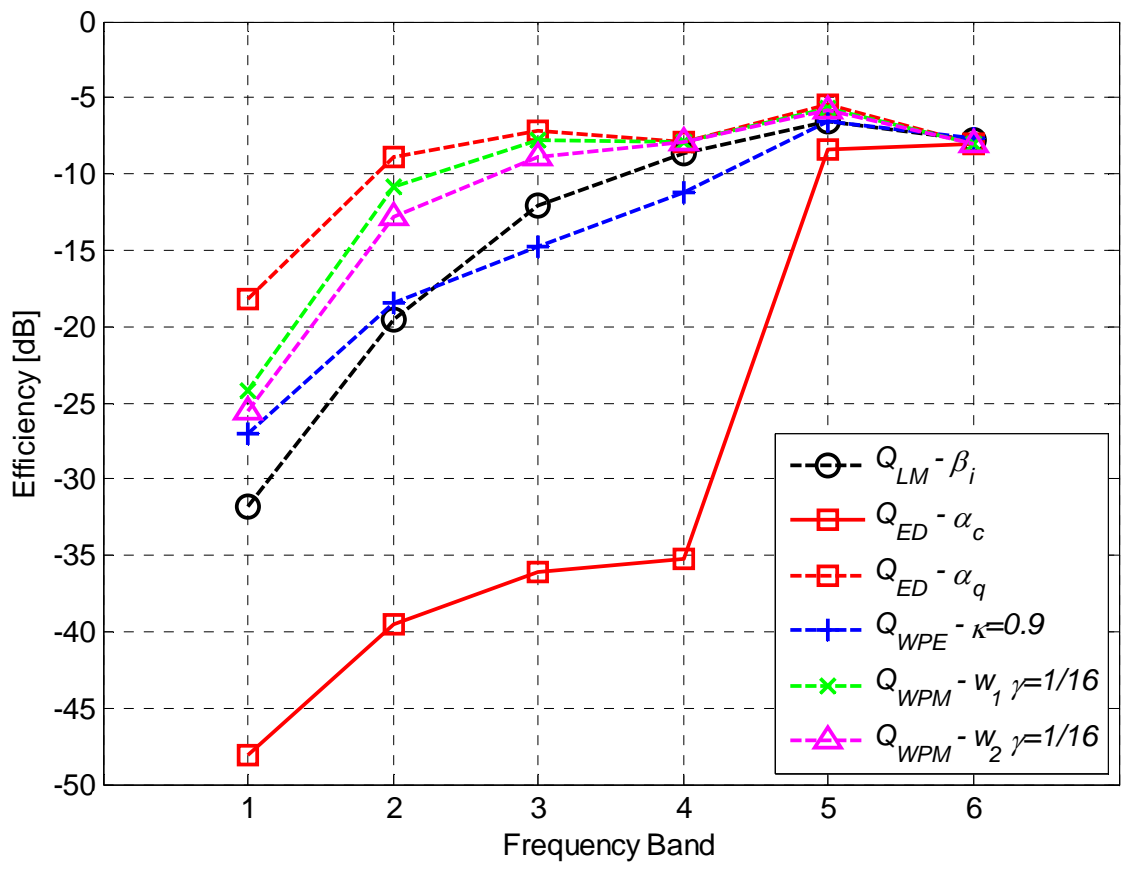

